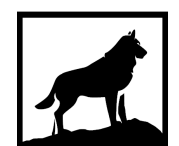

Michigan

Technological

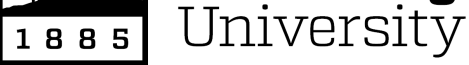

Michigan Technological University

Digital Commons @ Michigan Tech

DEVELOPMENT OF HIGH CAPACITY HYPERBRANCHED NITRIC OXIDE DONORS FOR CONTROLLING SUBCUTANEOUS INFLAMMATION

Sean Hopkins

Michigan Technological University, sphopkin@mtu.edu

Copyright 2015 Sean Hopkins

Recommended Citation

Hopkins, Sean, "DEVELOPMENT OF HIGH CAPACITY HYPERBRANCHED NITRIC OXIDE DONORS FOR CONTROLLING SUBCUTANEOUS INFLAMMATION", Open Access Dissertation, Michigan Technological University, 2015.

https://doi.org/10.37099/mtu.dc.etdr/22

Follow this and additional works at: https://digitalcommons.mtu.edu/etdr

Part of the Biomaterials Commons 


\title{
DEVELOPMENT OF HIGH CAPACITY HYPERBRANCHED NITRIC OXIDE DONORS FOR CONTROLLING SUBCUTANEOUS INFLAMMATION
}

\author{
By
}

Sean P. Hopkins

\begin{abstract}
A DISSERTATION
Submitted in partial fulfillment of the requirements for the degree of DOCTOR OF PHILOSOPHY

In Biomedical Engineering
\end{abstract}

MICHIGAN TECHNOLOGICAL UNIVERSITY

2015

(C)2015 Sean P. Hopkins 
This dissertation has been approved in partial fulfillment of the requirements for the Degree of DOCTOR OF PHILOSOPHY in Biomedical Engineering.

Department of Biomedical Engineering

Dissertation Advisor: $\quad$ Dr. Megan C. Frost

Committee Member: $\quad$ Dr. Bruce P. Lee

Committee Member: Dr. Jeremy Goldman

Committee Member: $\quad$ Dr. Jaroslaw Drelich

Department Chair: Dr. Sean J. Kirkpatrick 


\section{Table of Contents}

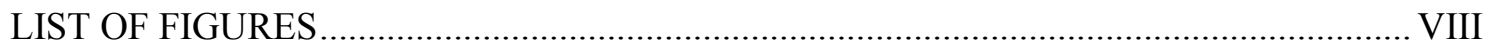

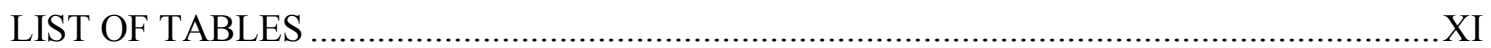

LIST OF ABBREVIATIONS ........................................................................................ XII

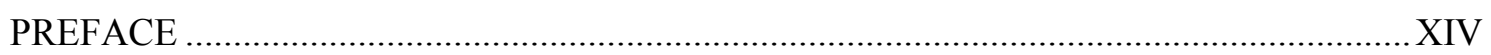

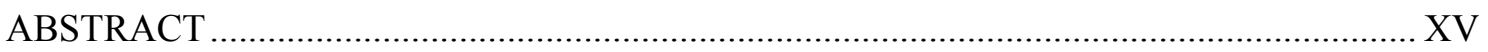

CHAPTER $1:$ INTRODUCTION .............................................................1

1.1 DISCOVERY AND BACKGROUND OF NITRIC OXIDE ......................................... 1

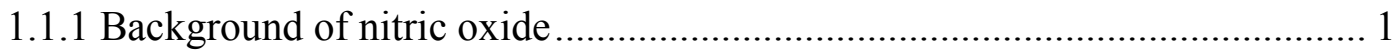

1.1.2 Nitric oxide's function in the body ........................................................... 1

1.2 BIOLOGICAL RESPONSE TO IMPLANTED BIOMATERIALS ............................ 5

1.2.1 Response to blood contacting foreign materials....................................... 5

1.2.2 Response to tissue contacting foreign materials....................................... 6

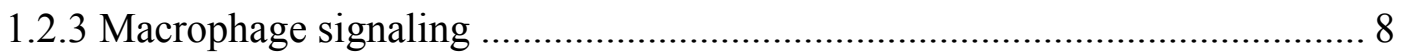

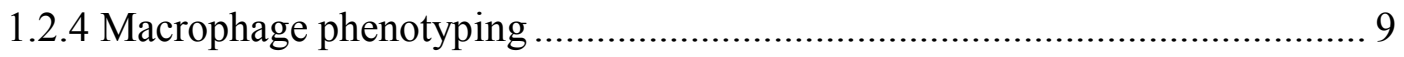

1.3 POLYMERS USEd IN BIOMEdiCAL SETTINGS ........................................... 10

1.3.1 Parameters for designing polymeric biomaterials ................................... 10

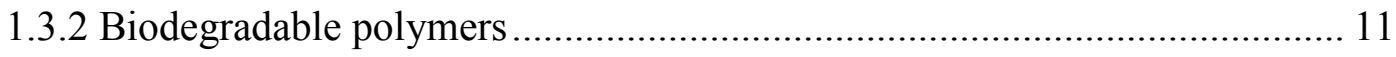

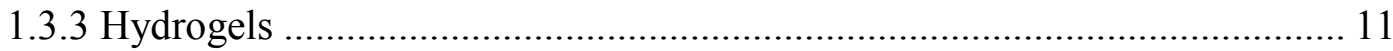

1.3.4 Hydrophobic polymers ....................................................................... 12

1.4 BaCkground of Current Nitric OXide Donating Materials........... 12

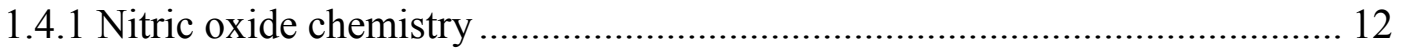

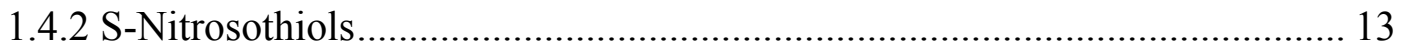

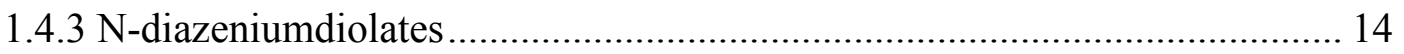

1.5 APPliCATIONS OF NitRic OXIDE DONATING MATERIALS ............................ 15

1.5.1. Concerns for nitric oxide concentration .............................................. 15

1.5.2 Improving biocompatibility of foreign devices .................................... 17

1.5.3 Interaction with macrophages and mast cells ......................................... 17 


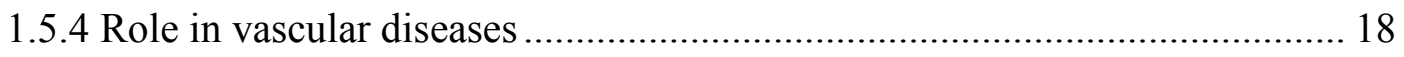

1.5.5 Cancer therapeutic applications.................................................................. 19

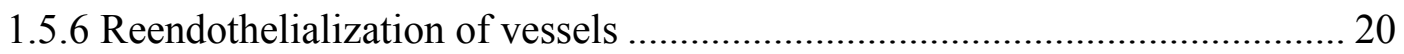

1.6 DETECTION METHODS OF NiTRIC OXIDE ....................................................... 20

1.6.1 Griess assay ........................................................................................ 20

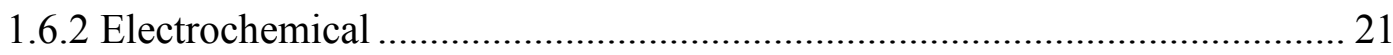

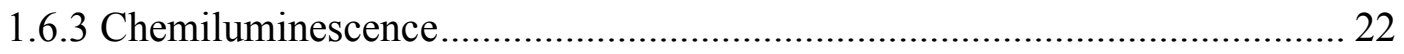

1.7 STATEMENT OF PURPOSE .................................................................................. 23

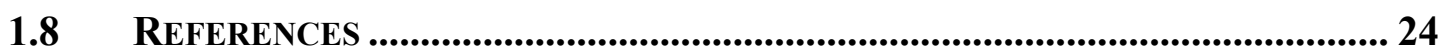

\section{CHAPTER 2 : CONTROLLED NITRIC OXIDE RELEASE FROM}

COVALENTLY LINKED S-NITROSO-N-ACETYL-DPENICILLAMINE TO POLYVINYL CHLORIDE (SNAP-PVC) .....36

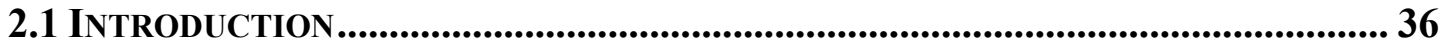

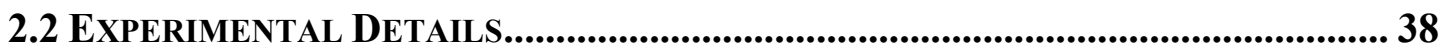

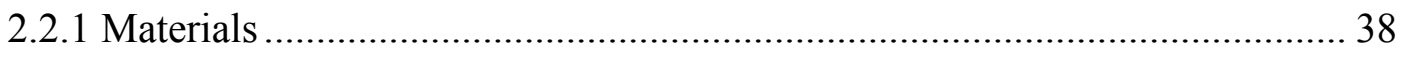

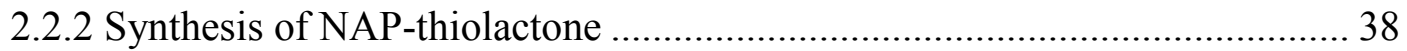

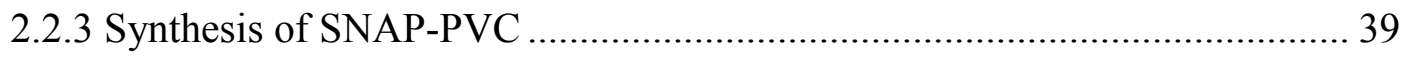

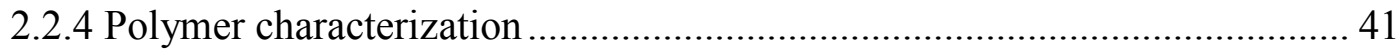

2.2.5 Quantification of primary amines............................................................... 42

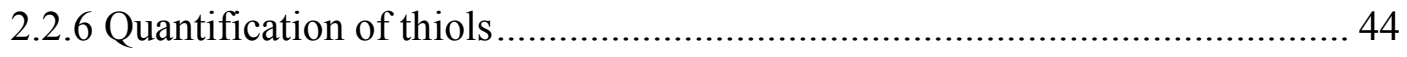

2.2.7 Nitric oxide release by photoinitiation ....................................................... 44

2.2.8 Nitric oxide measurements ......................................................................... 45

2.3 RESULTS AND DISCUSSION ....................................................................................... 45

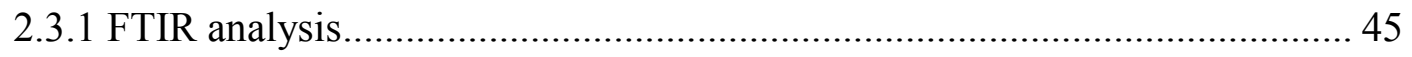

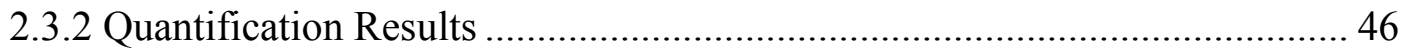

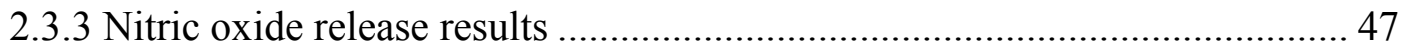

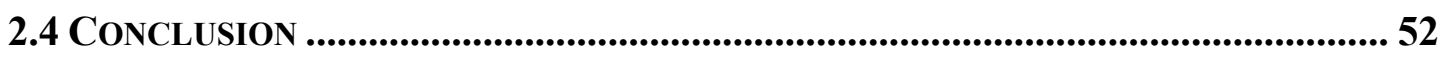

2.5 REFERENCES ................................................................................................ 52 
CHAPTER 3 : HIGH CAPACITY NITRIC OXIDE RELEASE FROM $S$-NITROSO- $N$-ACETYL-D-PENICILLAMINE MODIFIED

HYPERBRANCHED POLYAMIDOAMINE (SNAP-HPAMAM) FOR

CONTROLLED NITRIC OXIDE RELEASE .....................................56

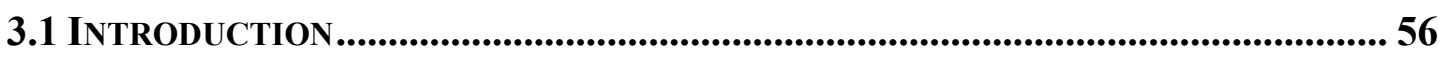

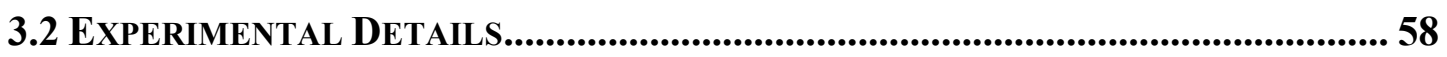

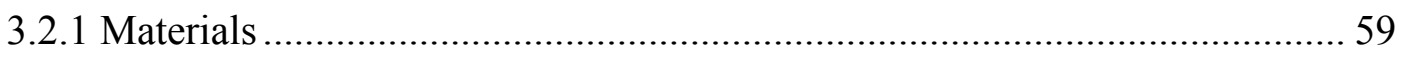

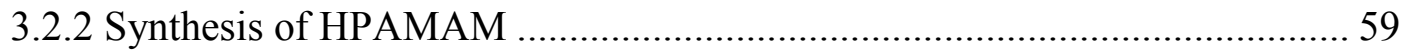

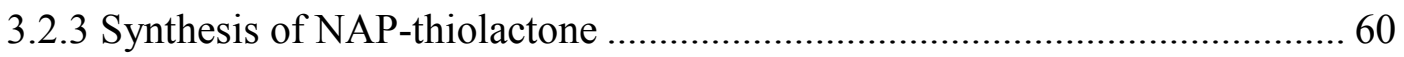

3.2.4 Nitrosation of N-acetyl-D-penicillamine modified HPAMAM ..................... 61

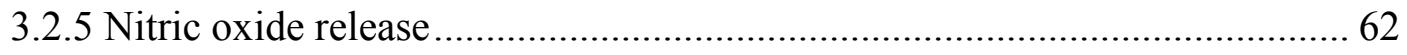

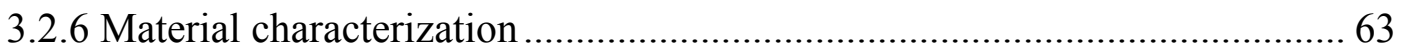

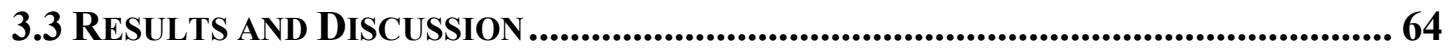

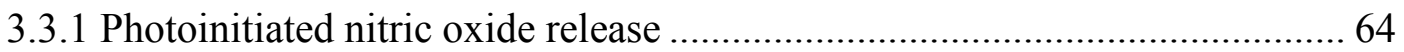

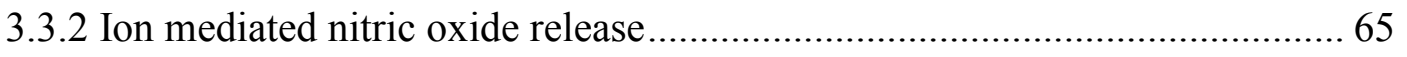

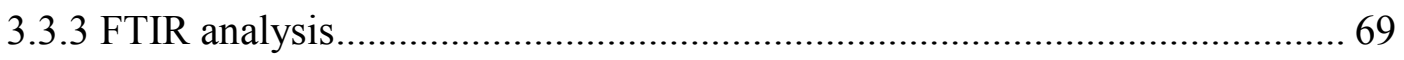

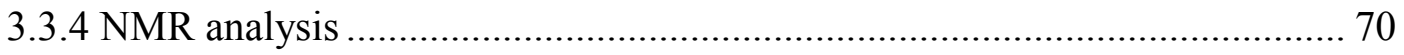

3.3.5 Quantification of thiols and primary amines ………………......................... 70

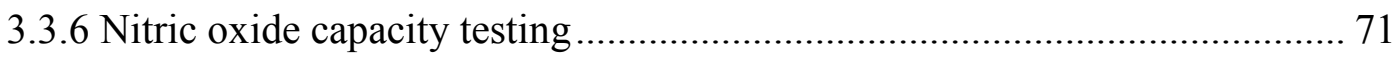

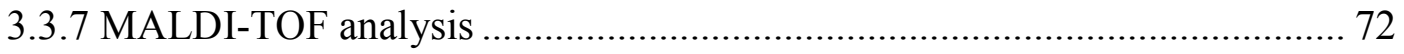

3.4 CONCLUSION …….................................................................................................. 74

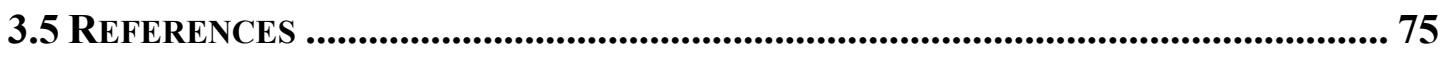

CHAPTER 4 : SUBCUTANEOUS INFLAMMATORY RESPONSE TO POLYVINYL CHLORIDE BASED NITRIC OXIDE

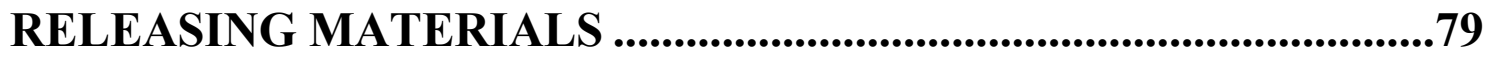

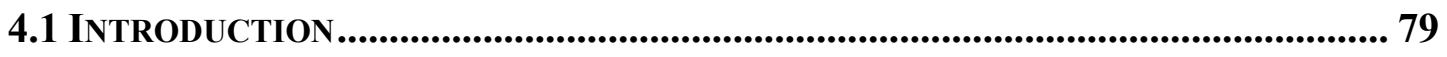

4.2 EXPERIMENTAL DETAILS............................................................................................ 81

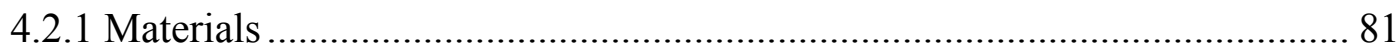


4.2.2 Synthesis of self-protected N-acetyl-D-penicillamine (NAP) thiolactone .... 82

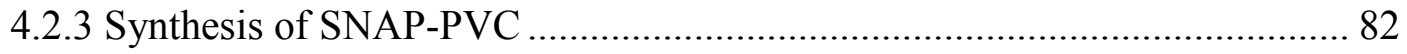

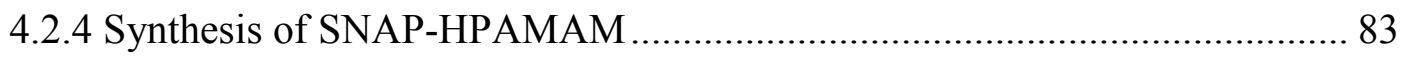

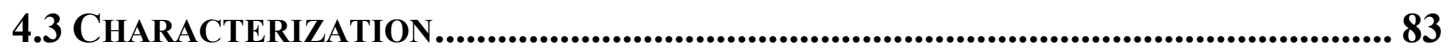

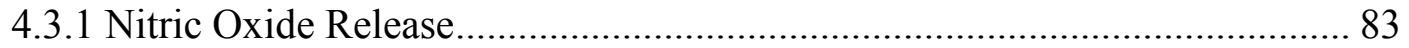

4.3.2 Polymer Film Casting and Implantation Procedure ................................. 84

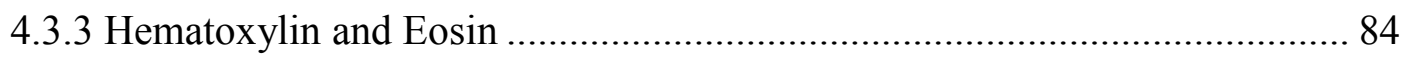

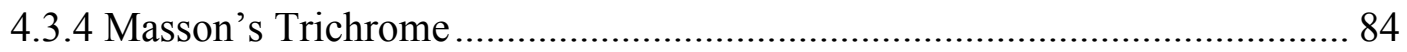

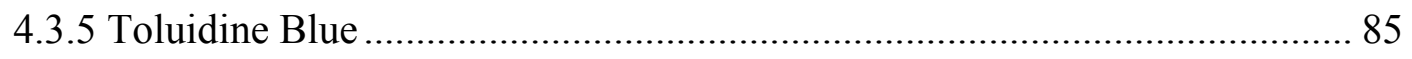

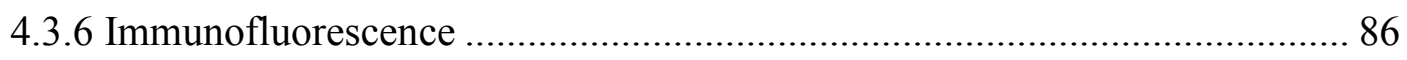

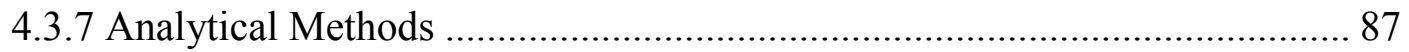

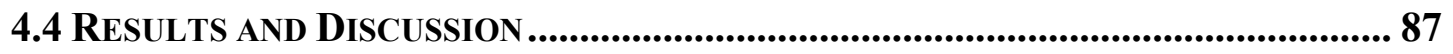

4.4.1 Polymer Functional Group Quantification ............................................. 87

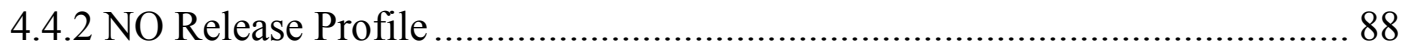

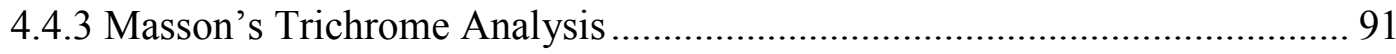

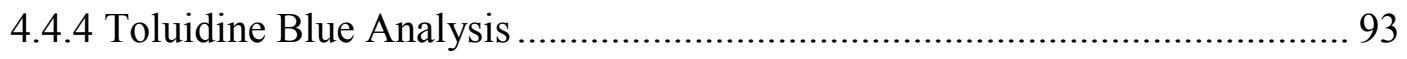

4.4.5 Hematoxylin and Eosin Analysis ..................................................... 94

4.4.6 Macrophage Immunofluorescence ....................................................... 95

4.4.7 Macrophage Phenotype Quantification .................................................. 97

4.5 CONCLUSION

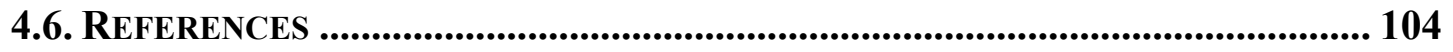

CHAPTER 5 : NITRIC OXIDE RELEASING MATERIALS AS

POTENTIAL ANTIVIRALS ...........................................................108

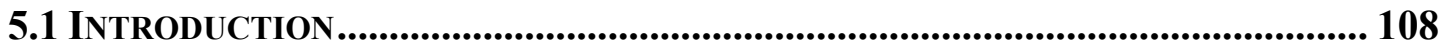

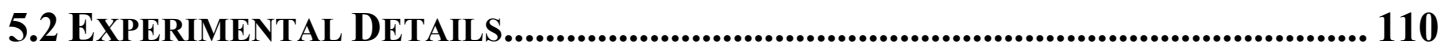

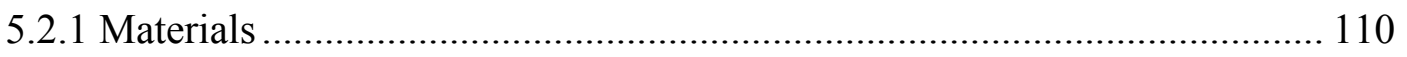

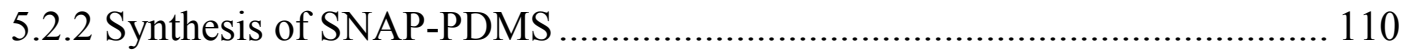

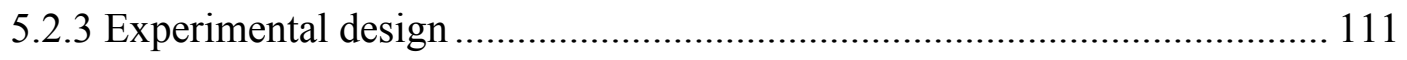

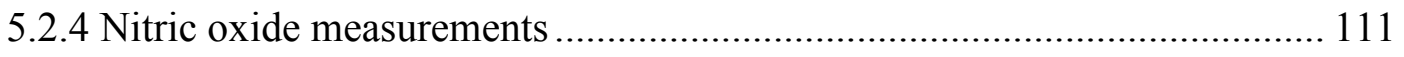


5.2.5 Virus activity measurements

5.3 RESULTS AND DISCUSSION ........................................................................... 112

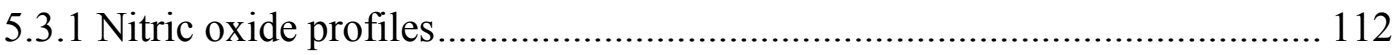

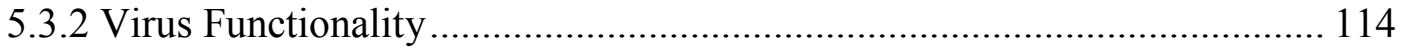

5.3.3 Preliminary Data Collection ............................................................ 114

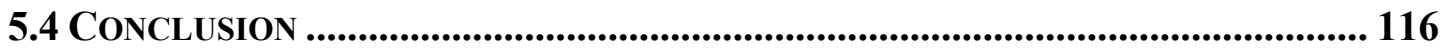

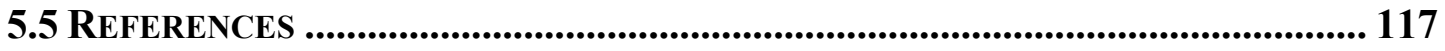

CHAPTER 6 : CONCLUDING REMARKS AND FUTURE

DIRECTION ....................................................................................................119

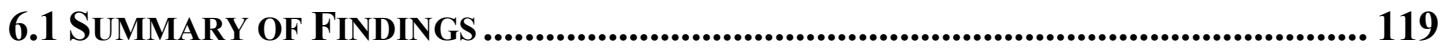

6.2 FUTURE DIRECTIONS ...................................................................................... 120

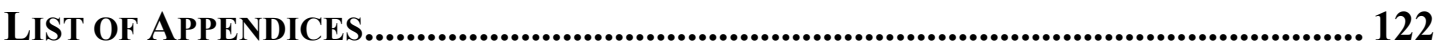




\section{List of Figures}

Figure 1.1. Mechanism for the production of NO from eNOS within endothelial cells. 3

Figure 1.2. Mechanism of production of NO at a neuronal synapse through nNOS.

Figure 1.3. Inducible production of NO through the regulation of iNOS within macrophages. 5

Figure 1.4. Biological response to implanted blood contacting biomaterials 6

Figure 1.5. Escalation of the inflammatory response and wound healing to an implanted biomaterial. 8

Figure 1.6. Structure of S-nitrosoglutathione (GSNO) and S-nitroso-N-acetyl-Dpenicillamine (SNAP). 14

Figure 1.7. Structure of dimethylhexanediamine diazeniumdiolates $\left(\mathrm{DMHD} / \mathrm{N}_{2} \mathrm{O}_{2}\right)$.

Figure 1.8. Chemical detection of NO through the Griess reaction........................... 21

Figure 1.9. Chemical mechanisms for the electrochemical detection of NO.......... 22

Figure 1.10. Mechanism for the detection of NO through chemiluminescence...... 23

Figure 2.1. Synthesis of thiolactone self-protected N-acetyl-D-penicillamine (NAP-

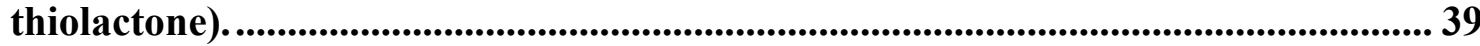

Figure 2.2. Synthesis route of SNAP-PVC. ................................................... 41

Figure 2.3. Mechanism for the fluorescent detection of primary amines using the ATTO-TAG FQ amine derivation kit............................................................... 43

Figure 2.4. Mechanism for the detection of free thiols using Ellman's assay......... 44 Figure 2.5. FTIR spectrum of PVC (green), PVC-NH2 (blue), and NAP-PVC (red). 46

Figure 2.6. Controlled NO release profile of SNAP-PVC at varying LED voltages. 48

Figure 2.7. NO release profile of SNAP-PVC placed in PBS at $37^{\circ} \mathrm{C}$. 49 
Figure 2.8. SNAP-PVC photoinitiated NO release at $37^{\circ} \mathrm{C}$ in air at different synthesis reaction times. 50

Figure 2.9. SNAP-PVC compounds with varied reaction times in EDA with triggered UV light photoinitiated for total NO release. 51

Figure 3.1. Synthesis schematic of hyperbranched polyamidoamine (HPAMAM). 60

Figure 3.2. Schematic of SNAP-HPAMAM's generic structure and triggered NO release methods. 63

Figure 3.3. NO release profile of SNAP-HPAMAM blended in PVC using photoinitiation. 65

Figure 3.4. Passive NO release from $2.16 \mathrm{mg}$ of SNAP-HPAMAM in $2 \mathrm{~mL}$ of PBS at $37^{\circ} \mathrm{C}$ 66

Figure 3.5. Passive NO release of $6.01 \mathrm{mg}$ of SNAP-HPAMAM encapsulated in $\mathrm{PVC}$ in PBS at $37^{\circ} \mathrm{C}$ 68

Figure 3.6. Passive NO release in $\mathrm{PBS}$ at $37^{\circ} \mathrm{C}$ from multiple polymers blended with SNAP-HPAMAM. 69

Figure 3.7. FTIR spectrum of hyperbranched compounds. ..................................... 70

Figure 3.8. MALDI-TOF analysis of HPAMAM............................................... 72

Figure 3.9. MALDI-TOF analysis of NAP-HPAMAM. ........................................ 73

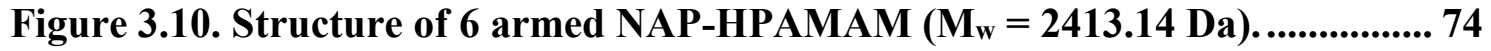

Figure 4.1. Layered polymer schematic for passive NO release when implanted subcutaneously 89

Figure 4.2. NO release profile of SNAP-PVC in PBS at $37^{\circ} \mathrm{C}$............................... 90

Figure 4.3. NO release profile of SNAP-HPAMAM PVC in PBS at $37^{\circ} \mathrm{C}$............. 91

Figure 4.4. Trichrome analysis of 15 day subcutaneously implanted materials..... 92 Figure 4.5. Fibrous encapsulation thickness data for PVC, SNAP-PVC, and SNAPHPAMAM PVC for 15 day implants.............................................................. 93

Figure 4.6. Difference between active and inactive mast cells............................... 94

Figure 4.7. H\&E analysis of 15 day subcutaneously implanted materials............. 95 
Figure 4.8. Cell culture of RAW 264.7 murine macrophages stained with iNOS at $600 x$. 99

Figure 4.9. Immunofluorescence of iNOS $^{+}$cells (green) around 15 day implants.100 Figure 4.10. Inflammatory response to polymer fragments seen in 15 day PVC implants. 101

Figure 4.11. Immunofluorescence and analysis of cell nuclei, CD11b, and CD163 marked cells for 15 day implants. 102 Figure 5.1. Mechanism for DNA damage within viral species from an NO donating material. 109

Figure 5.2. NO release profile of SNAP-PDMS over 2 hours when LED activated. 113

Figure 5.3. NO release profile of SNAP-PDMS passively with no LED over 2 hours. 113

Figure 5.4. NO release profile from SNAP-PVC over a 2 hour time period......... 114

Figure 5.5. PPV removal using SNAP-PVC as the NO releasing polymer. 115 Figure 5.6. PPV removal using pre-washed SNAP-PVC as the NO releasing polymer 116 


\section{List of Tables}

Table 1.1. Effect of NO when administered at varying concentrations to certain cell

types.

Table 2.1. FQ and CHN analysis on aminated PVC to observe the primary amine

and nitrogen content as reaction time with ethylenediamine increases. ................ 52

Table 4.1. Quantification of important functional groups and NO capacity of

SNAP-PVC and SNAP-HPAMAM as each reaction step progresses....................... 88 


\section{List of Abbreviations}

$\mathrm{CaM}$ calmodulin

cGMP. cyclic guanosine monophosphate

CHCA $\alpha$-cyano-4-hydroxycinnamic acid

DAPI 4',6-diamidino-2-phenylindole

DEHP bis(2-ethylhexyl) phthalate

DETA diethylenetriamine

DMAC N,N-dimethylacetamide

DTNB 5,5-dithio-bis-(2-nitrobenzoic acid)

ECC extracorporeal circuit

ECM extracellular matrix

EDA ethylenediamine

EDRF endothelial relaxation factor

eNOS endothelial nitric oxide synthase

FBGC foreign body giant cell

FTIR .Fourier transform infrared spectroscopy FQ 3-2-(furoyl quinoline-2-carboxaldehyde)

GSH glutathione

GSNO S-nitrosoglutathione

GTP guanosine-5'-triphosphate

$\mathrm{H} \& \mathrm{E}$ hematoxylin and eosin

HPAMAM hyperbranched polyamidoamine IFN. interferon

IL interleukin

iNOS inducible nitric oxide synthase

$\mathrm{KCN}$ potassium cyanide

LDL low density lipoprotein

LED light emitting diode

LPS lipopolysaccharide 
MALDI-TOF MMP matrix assisted laser desorption/ionization time of flight

$\mathrm{NADPH}$ nicotinamide adenine dinucleotide phosphate

NAP

NMR

nNOS

NO

NOA

NONOate

NOS

$\mathrm{ONOO}^{-}$

PAMAM

PBS

PDGF.

PDMS

PEG.

PLLA .

PTFE

PVC

ROS

RSNO

sGC

SNAP

TGF

TNB

TNF

TWEEN

VEGF matrix metalloproteinases N-acetyl-D-penicillamine nuclear magnetic resonance neuronal nitric oxide synthase nitric oxide nitric oxide analyzer diazeniumdiolate nitric oxide synthase peroxynitrite ..polyamidoamine phosphate buffered saline platelet derived growth factor .polydimethylsiloxane .polyethylene glycol .. poly-1-lactic acid polytetrafluoroethylene polyvinyl chloride reactive oxygen species S-nitrosothiol guanylate cyclase S-nitroso-N-acetyl-D-penicillamine tissue growth factor 2-nitro-5-thiobenzoate tissue necrosis factor polysorbate 20 vascular endothelial growth factor xiii 


\section{Preface}

Chapters 2,3, and 4 are in the process of being submitted to peer-reviewed journals and are reproduced here. I am the primary author for the papers and performed the majority of the experiments for all of the chapters.

In chapters 2, 3, and 4, all of the experimental work was performed by me and the analytical work by both my advisor, Dr. Megan Frost, and myself. The writing for all of the publications was also guided by Dr. Megan Frost.

The analysis and histology was performed by me for chapter 4 . The surgeries were performed by Dr. Jeremy Goldman. Roger Guillory helped perform some of the staining done in chapter 4, specifically the toluidine blue, hematoxylin and eosin, and Masson's trichrome stains. Weilue He performed any cell culture experiments involving macrophage growing and differentiating.

The background information given in chapter 1 is unpublished background material and the virus culture, inoculation, and analysis seen in chapter 5 was performed by Eric Pearson from Dr. Caryn Heldt's lab in the chemical engineering department while I constructed the materials and lightboard used during experimentation. The data obtained in this chapter is strictly preliminary and will not be published in the future. Betsy Kruppe in the biomedical engineering department will be taking over the future experimentation of this project. 


\begin{abstract}
Implanted medical devices undergo complications the longer they remain in contact with tissue or blood. This rejection of foreign materials by our body is one of the largest reasons innovations in biomedical sensors and implanted technology are being held back. One means to hold off this unwanted response is through the utilization of nitric oxide (NO) releasing materials. Two unique NO releasing polymeric materials were synthesized and characterized before being implanted subcutaneously. Both NO releasing materials described used S-nitrosothiol (RSNO) chemistry as the main mechanism for NO release. The first material described covalently links an RSNO to the backbone of PVC while the second material has RSNOs covalently attached to a hyperbranched polyamidoamine (HPAMAM) molecule, which is then blended within a polymer matrix. A high reservoir of NO was observed in the NO releasing HPAMAM when compared to other $\mathrm{NO}$ releasing polymers.
\end{abstract}

The two materials (SNAP-PVC, SNAP-HPAMAM blended in PVC) were implanted subcutaneously and were tested versus control polymers that did not release $\mathrm{NO}$; materials were explanted after 1 and 15 days and histological characterization was completed. The inflammatory response was then observed through histological analysis and NO demonstrated anti-inflammatory properties, specifically by observing the presence of cells marked with CD11b, CD163, and iNOS. Fibrosis was also a major inflammatory response carefully observed. NO releasing implants showed a much more resolved state of inflammation and wound healing while the controls demonstrated signs of chronic inflammation and increased number of pro-inflammatory cells. The long lasting SNAP-HPAMAM PVC NO releasing materials showed a large reduction in chronic inflammatory macrophages marked with iNOS with a slight upregulation in anti-inflammatory macrophages after 15 days of implantation. Compared to control PVC implants, a significant reduction in fibrosis was observed as the encapsulation thickness was $120.28 \pm 36.1 \mu \mathrm{m}$ while SNAP-PVC was $74.20 \pm 29.9 \mu \mathrm{m}$ and SNAPHPAMAM was $38.68 \pm 21.0 \mu \mathrm{m}$. A trend was seen in the reduction of CD11 $\mathrm{b}^{+}$cells with 
an increase in NO from the implants, along with an increasing trend of $\mathrm{CD}_{163^{+}}$cells. The presence of chronic inflammatory iNOS cells was also greatly reduced with the increase of NO to the surrounding subcutaneous tissue. 


\section{Chapter 1 : Introduction}

\subsection{Discovery and Background of Nitric Oxide}

\subsubsection{Background of nitric oxide}

The first role of nitric oxide (NO) as an important signaling molecule was initially demonstrated by Ferid Murad in $1977^{1}$. Murad and his colleagues investigated the impact NO had on enzyme regulatory functions within the body- specifically with guanylate cyclase (sGC) and the formation of cyclic guanosine monophosphate (cGMP). Once this mechanism of activation was clarified, the role of cGMP was then studied more closely. They investigated the role of nitrite containing compounds within various organs of the body and observed vascular relaxation and NO were closely associated, but did not exactly understand the mechanism that was responsible for this phenomenon $^{1-2}$. The discovery of endothelial cells impact on controlling the relaxation of smooth muscle cells through acetylcholine was then proven by Robert F. Furchgott, bringing up the role of endothelial derived relaxation factor (EDRF) within vascular endothelial cells ${ }^{3}$. The identity of this unknown vasorelaxation function was then proven to be NO in 1986 when he and Louis J. Ignarro along with other colleagues proved that the EDRF that was causing smooth muscle cell relaxation was actually $\mathrm{NO}^{4-}$ 5 . Identifying this important pathway brought up a multitude of other questions of its function in various places throughout the body. Virtually every mammalian cell in the body is influenced in some way by NO as this highly reactive free radical molecule plays an important role in cell signaling.

\subsubsection{Nitric oxide's function in the body}

The production of NO is accomplished through specific enzymatic pathways based on three different isoforms depending on the location in the body: endothelial nitric oxide synthase (eNOS), neuronal nitric oxide synthase (nNOS), and inducible nitric oxide 
synthase (iNOS). These enzymes produce NO by catalyzing the reaction of L-arginine and nicotinamide adenine dinucleotide phosphate (NADPH) to form L-citrulline and $\mathrm{NO}^{6-7}$. eNOS and nNOS are labeled as constitutive NOS as they are calcium-calmodulin dependent pathways. Healthy vasculature requires the production of NO through eNOS within endothelial cells ${ }^{8-9}$. Under normal conditions, NO is constantly produced by eNOS within blood vessels. Stimulation of NO synthesis begins either through shear stress caused by blood flow along the endothelial lining or by endothelial receptors specific to certain ligands which stimulate calcium release within the cell to eventually activates $\operatorname{eNOS}^{10}$ (Figure 1.1). NO produced diffuses into the smooth muscle cell layer to activate the enzyme sGC. sGC is then modified into cGMP, which is an important factor for causing smooth muscle cell relaxation and vasodilation within blood vessels $^{11}$. NO is also released into the bloodstream where it interacts with platelets, contributing to the non-adhesive properties to vasculature through the same sGC-cGMP pathway seen in smooth muscle cells ${ }^{12-13}$. If NO production is impaired in the endothelium, the risk for atherosclerosis and hypertension drastically increase. Neuronal cell signaling is the primary function of NO production through nNOS (Figure 1.2). The presence of NO was discovered in numerous neural systems in the body, which regulate the release of certain neurotransmitters such as the increase in production of acetylcholine ${ }^{14,15}$. iNOS is the one isoform that is not dependent on the calciumcalmodulin pathway that eNOS and nNOS require. This is due to the fact that the calmodulin is more tightly bound when compared to the other enzymes, and is unable to react as readily with calcium ions ${ }^{16}$. Macrophages are the main producer of iNOS, and are activated in the presence of certain cytokines and bacteria ${ }^{17}$ (Figure 1.3). It is a strong mediator of inflammatory response and regulating the immune system. The NO produced in this pathway is used to destroy foreign bacteria as NO forms peroxynitrites $\left(\mathrm{ONOO}^{-}\right)$in the presence of radical oxygen species (ROSs), a highly damaging product to cellular structures ${ }^{18}$. 


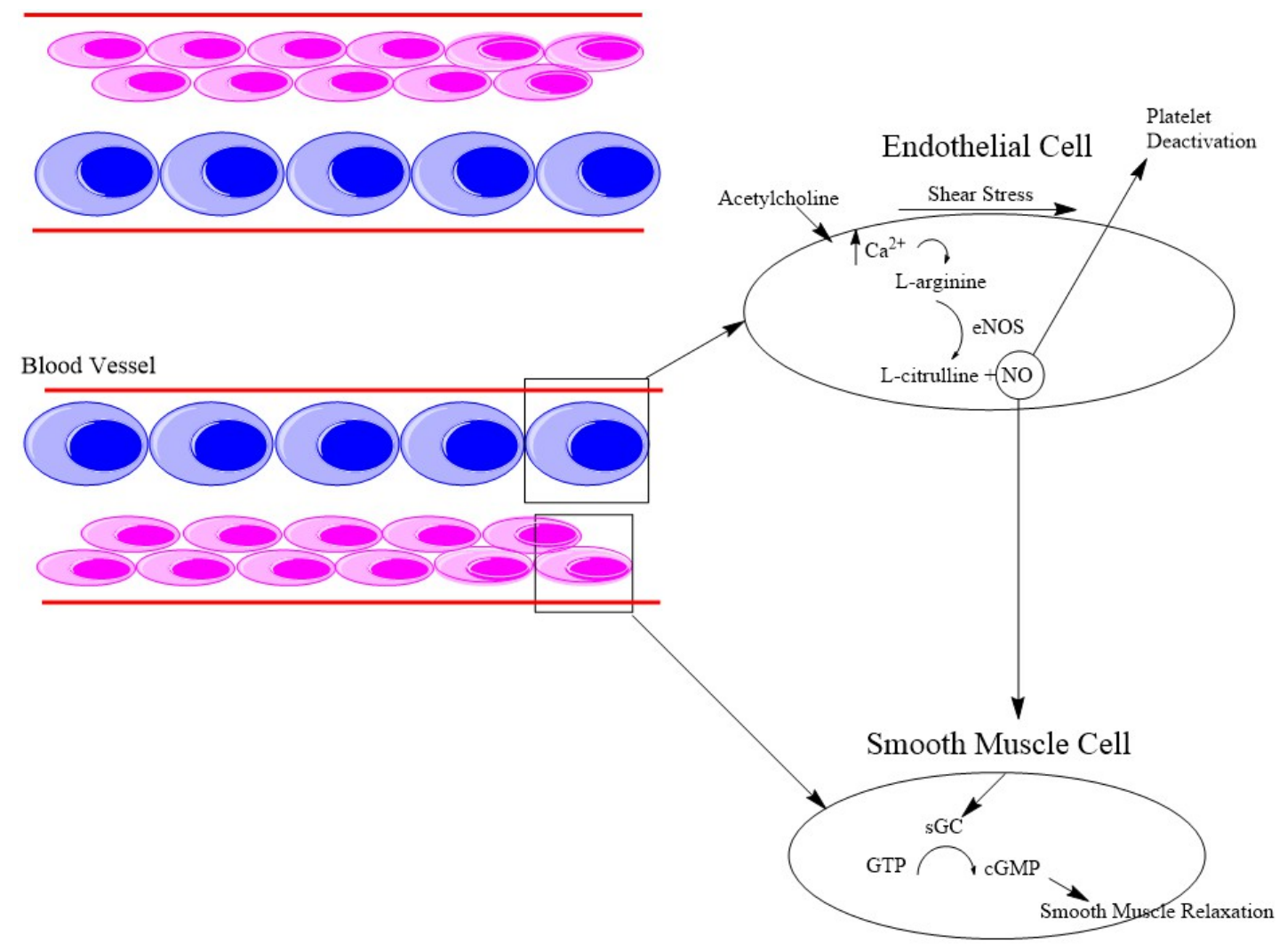

Figure 1.1. Mechanism for the production of NO from eNOS within endothelial cells. Acetylcholine and/or shear stress causes the upregulation of calcium ions within endothelial cells, which leads to the conversion of L-arginine to L-citrulline and NO facilitated by eNOS. NO then diffuses into the bloodstream where it causes platelet deactivation and into the smooth muscle layer where vasorelaxation occurs. 


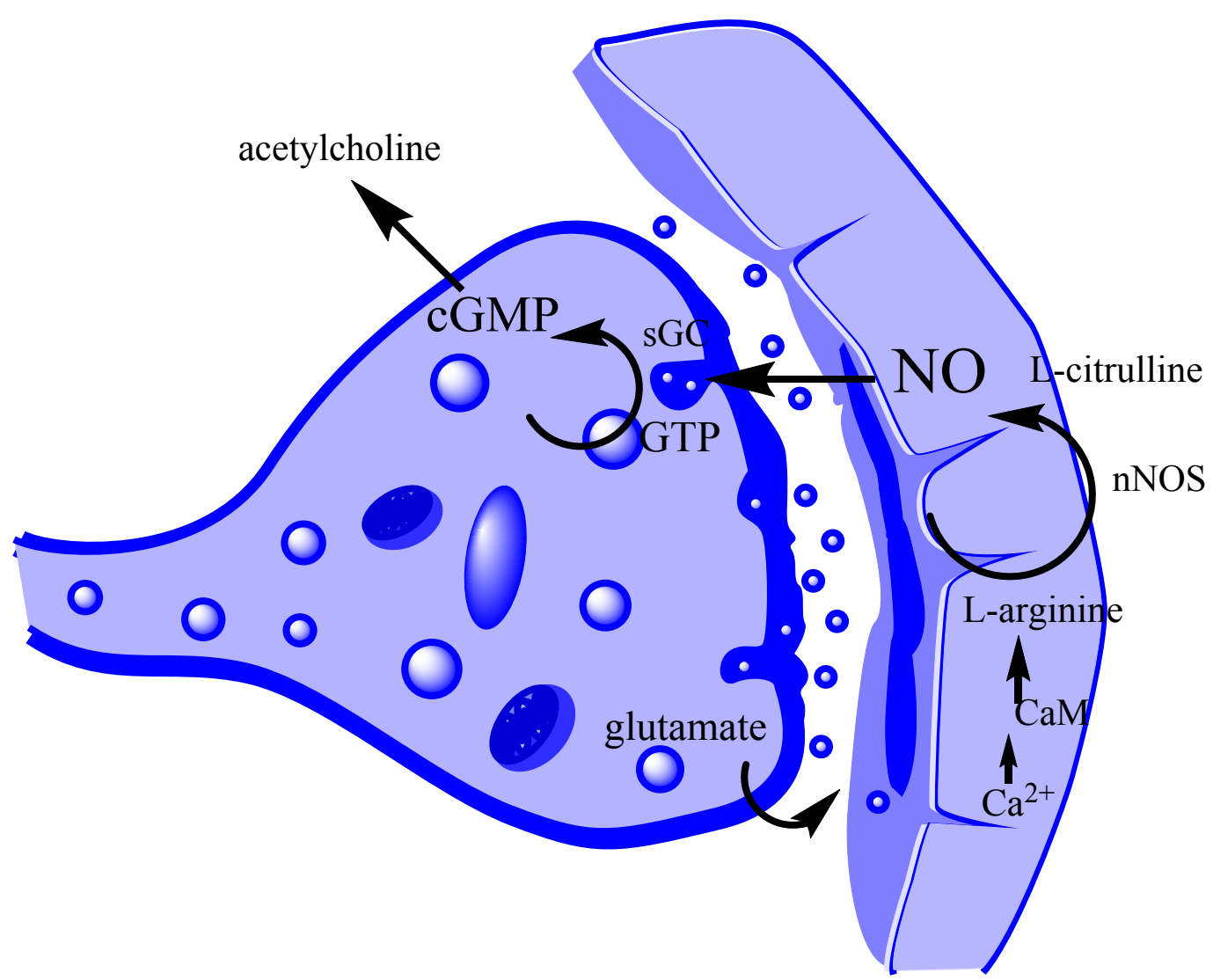

Figure 1.2. Mechanism of production of NO at a neuronal synapse through nNOS. The production is first stimulated by the release of glutamate to cause the upregulation of calcium within the connecting neuron. This then leads the conversion of L-arginine to L-citrulline and NO facilitated by nNOS. NO then leads to the production of cGMP which regulates ion mediation in neurons along with controlling the presynaptic release of certain neurotransmitters like acetylcholine. 


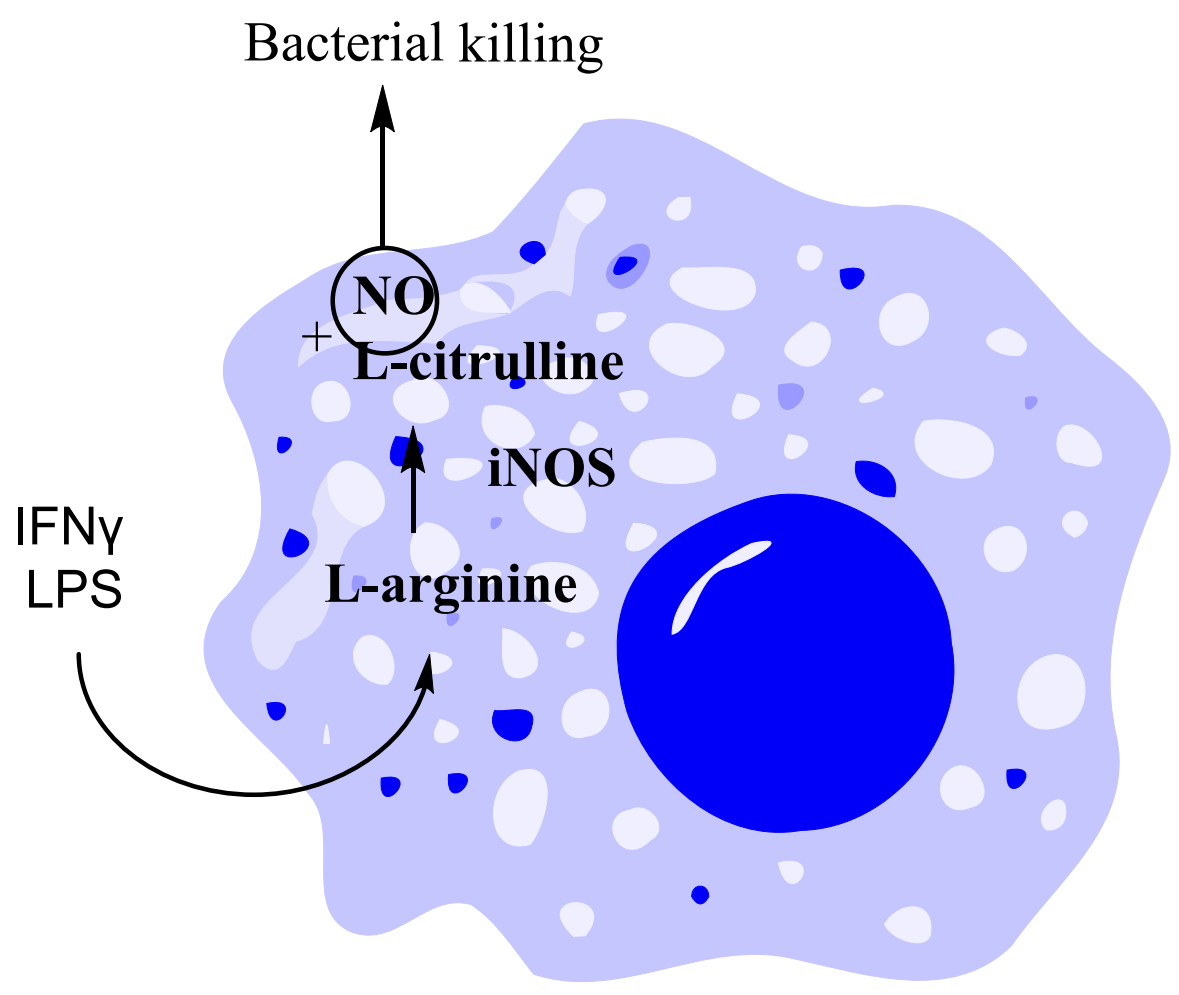

Figure 1.3. Inducible production of NO through the regulation of iNOS within macrophages. The only NO pathway that is not calcium-calmodulin regulated. It is triggered by the interaction of macrophages with specific cytokines (IFN $\gamma$ ) and bacterial products (LPS). After NO is produced, its main role in this instance is the intracellular killing of pathogen through the formation of $\mathrm{ONOO}^{-}$.

\subsection{Biological Response to Implanted Biomaterials}

\subsubsection{Response to blood contacting foreign materials}

There is huge potential to improve patient interventions by controlling the biological response toward blood and tissue contacting devices and the materials in which they are fabricated. The response from the body when a foreign material is introduced to the biological environment often times causes failure of the implanted device. In the case of intravascular implants such as sensors and catheters, proteins immediately adsorb to the surface of the material upon implantation, which platelets then bind to and become activated (Figure 1.4). Once activated, soluble fibrinogen is converted to insoluble 
fibrin, forming a clot ${ }^{19-20}$. This can cause serious complications for the device that is implanted inside a patient such as incorrect sensor readings or occluded catheters due to the interference around the sensor area or the lumen of the catheter. Clot formation also creates a risk for embolism as it can break off of the device and block a vessel further down the bloodstream.

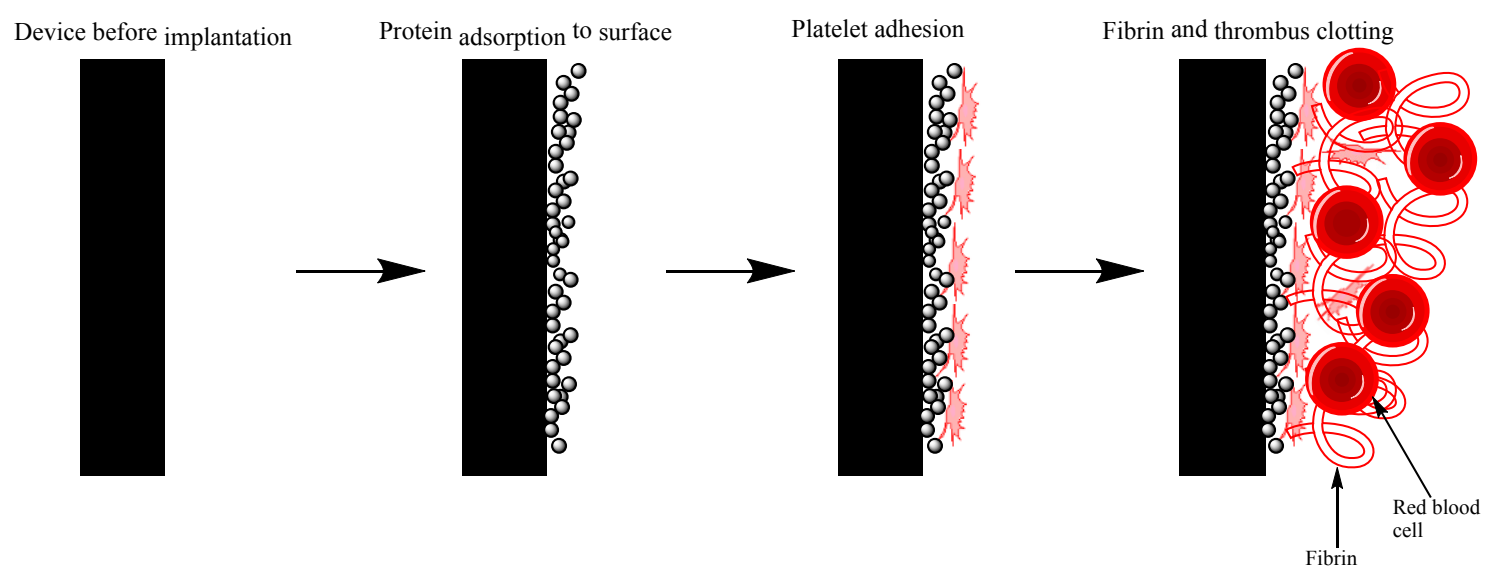

Figure 1.4. Biological response to implanted blood contacting biomaterials. Once a foreign material comes in contact with blood, proteins will immediately adhere to the surface. This creates binding sites for circulating platelets to attach to and become activated. After platelet activation, fibrin is formed which leads to even more platelet adhesion to the surface and eventually a thrombus is formed.

\subsubsection{Response to tissue contacting foreign materials}

Medical devices that are implanted within the tissue of a patient will undergo a wound healing response. The inflammatory response is an important part of the wound healing process in the body, and work together to contain/isolate or remove foreign materials while remodeling and repairing any tissue damage that occurred around the device. This response can have negative effects on device functions. One common issue that leads to their failure is the formation of a fibrous capsule around the implant along with chronic inflammation occurring due to the constant recruitment of macrophages to the area ${ }^{21}$. If a material that is unable to be properly phagocytized by the converging macrophages, it is completely isolated from the rest of the body with a thick collagenous capsule. Over time, this can cause a complete loss of functionality of the implant, requiring it to be 
replaced which subjects the patient to more invasive surgeries. This response is due to the macrophages present around the implant area attempting to remodel the damaged extracellular matrix (ECM). During this remodeling phase, an overexpression of fibronectin by macrophages is present along with the release of pro-fibrogenic cytokines to increase fibroblast activation ${ }^{22-23}$. Macrophages release matrix metalloproteases (MMPs) to facilitate the remodeling, which influence how other cells migrate to the foreign material and differentiate ${ }^{24}$.

The evaluation of the biocompatibility of a material is often gauged through histological analysis over a period of time. The inflammatory response is a complex series of signaling chemicals and cells that vary in quantity and type (Figure 1.5). Neutrophils and other emigrating white cells are predominant during the acute inflammation stage and only last for a few hours to days, but macrophages have also been shown be in their highest concentration while neutrophils are still present ${ }^{25}$. This migration of leukocytes to the injury site is facilitated through the chemotaxis process. A large variety of chemotactic species are released from the body and attach to receptors on the leukocyte cell membranes which causes them to permeate through the vasculature to the wound site and become activated. Once activated, neutrophils begin to phagocytize any foreign materials by attaching itself to it, engulfing it, and eventually degrading or killing it ${ }^{26}$. If the initial cause of inflammation is not resolved during the acute phase, chronic inflammation begins. This is stimulated from a constant release of pro-inflammatory stimuli that occur during the acute inflammatory phase. During the chronic inflammation phase, the presence of neutrophils drastically decreases, and macrophages, monocytes, and lymphocyte occurrence increases ${ }^{27}$. There is no longer a systemic response as the foreign body response is limited to the injury site or biomaterial. 


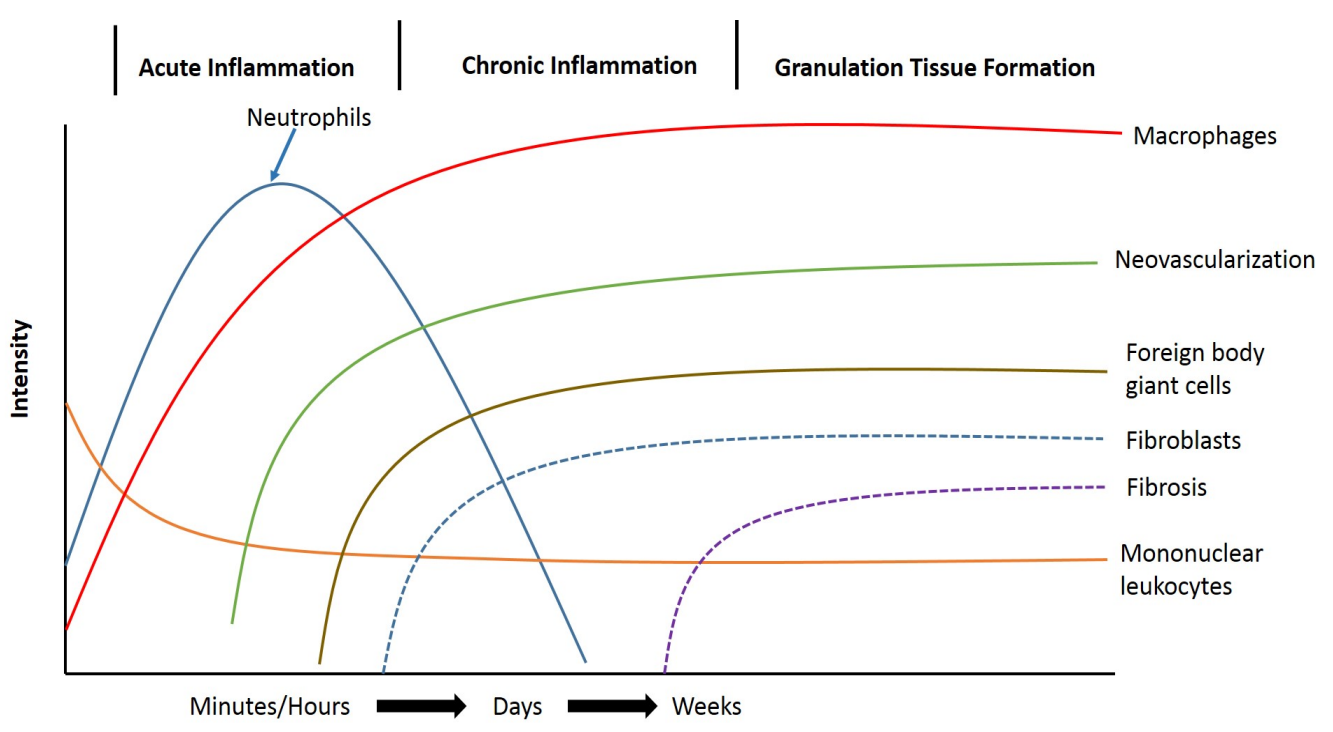

Figure 1.5. Escalation of the inflammatory response and wound healing to an implanted biomaterial. Time and intensity vary on the chemical and physical properties of implanted biomaterial and the degree of trauma caused during implantation. Neutrophils and blood monocytes first migrate to the injury site, then within hours, the blood monocytes are converted into macrophages. Once chronic inflammation occurs, neutrophils are removed by the macrophages present and eventually form FBGCs to remove large particulates. The final resolution is to isolate off the material through a fibrous capsule. Modified figure previously done by Anderson ${ }^{26}$.

\subsubsection{Macrophage signaling}

Macrophage migration and adhesion to foreign biomaterials is the inevitable host response that causes the most complications for biological implants. For blood contacting materials, platelets will release a number of chemoattractants like interleukin (IL-1), transforming growth factor (TGF- $\beta$ ), and platelet-derived growth factor (PDGF) to allow macrophages to reach the implantation site ${ }^{28}$. Once macrophages arrive, more chemoattractants such as PDGF, tumor necrosis factor (TNF- $\alpha$ ), and IL-6 are released from the macrophages themselves to recruit even more to the site ${ }^{28}$. Implants that are left in long enough will eventually lead to the formation of foreign body giant cells (FBGCs). Macrophages that are adhered to the biomaterial surface are signaled by IL-4 and IL-13 to initiate the fusion into FBGCs if the material is too large to be phagocytized $^{29-31}$. Surface modifications of implanted polyurethanes have been shown 
to alter the way macrophages adhere and fuse to form FBGCs by introducing silicone into the polymer matrix showing the possibility to control chronic inflammation on a material ${ }^{32}$. However, once FBGCs are formed, the process of frustrated phagocytosis begins. This process occurs as an attempt to degrade and destroy the foreign substance that was unable to be removed simply by macrophages. FBGCs will release ROSs, degradative enzymes, and acid hydrolases at the material surface in an attempt to remove $\mathrm{it}^{33-34}$. The introduction of ROSs to the environment allows for alterations in surface chemistry of the implanted material, leading to an even greater loss of material functionality.

\subsubsection{Macrophage phenotyping}

Macrophage phenotype also plays a large role in the inflammatory response to biomaterials and are labeled as either classically activated macrophages, or M1 macrophages, and alternatively activated macrophages, or M2 macrophages. M1 macrophages are activated from the presence of bacterial lipopolysaccharides and signaling chemicals related with infection ${ }^{35}$. This phenotype is strongly associated with the aggressive removal of foreign substances through inflammation and are responsible for the release of pro-inflammatory cytokines like IL-1 $\beta$, IL-6, IL-12, and TNF- $\alpha^{36}$. M2 macrophages are more involved with tissue repair and dealing with parasitic types of infections ${ }^{37}$. Instead of increasing the amount of inflammatory cells to be recruited to a site, this phenotype releases anti-inflammatory cytokines such as TGF- $\beta$ and IL-10 to help control $\mathrm{it}^{36}$. The two phenotypes also have very different metabolic pathways of Larginine as well. As described with the NOS enzyme reactions, L-arginine is an important precursor for the production of NO. The production and utilization of NO through iNOS is mainly done through M1 macrophages as a means to initiate intracellular killing to foreign bacteria. M2 macrophages upregulate arginase to synthesize urea and ornithine which are important for the production of collagen and cell proliferation $^{38-39}$. These mechanisms make sense when applied to a typical wound healing situation, where M1 macrophages are dominant for the first few days to remove 
foreign substances and then M2 macrophages take over after to remodel and repair the damaged tissue $e^{40-41}$.

\subsection{Polymers Used in Biomedical Settings}

\subsubsection{Parameters for designing polymeric biomaterials}

Polymers are one of the most widely used materials in medicine. Their applications are limitless due to their highly modifiable mechanical and chemical properties which allow them to be designed for specific situations. Depending on the application a polymer is needed, there are certain criteria that must be met to ensure the best results ${ }^{42}$. Implanted polymers could be required to be a facilitator of mass transfer from a device to the surrounding tissue. This brings up the issue of porosity of a polymer into question. If the polymer is transporting large molecules like proteins, it must have a tailored permeability to allow that transfer. Other types of polymers only need to facilitate small gaseous molecules like oxygen, and have more lenient porosity parameters. Some common biocompatible polymers used for limited molecular permeability of water and other larger molecules are silicone rubbers and polytetrafluoroethylene (PTFE) ${ }^{43-44}$.

Reactivity is another important parameter that must be taken into consideration for an implanted polymer. When in contact with tissue, the cells involved in the foreign body response will release harsh reactive chemicals in an attempt to degrade it. The surface of a biomaterial will become acidic as a result of this response, sometimes reaching a $\mathrm{pH}$ as low as $4^{45}$. A polymer must be able to resist this reactive environment as the inflammatory response progresses. In the late 1980's, polyether polyurethane elastomers were used for pacemaker lead insulation over silicone rubber due to their better mechanical and chemical properties. Over time, device failure occurred due to environmental stress cracking within the polymer. This occurred due to chemical ion oxidation that led to the cleavage of the polymer chains, causing a reduction in mechanical properties ${ }^{20}$. Chemical modifications are a popular method to make polymers relatively inert in the body to prevent this type of complication. Some 
polymers have reactive functional groups allowing for the attachment of drugs or proteins to increase the biocompatibility and lower the reactivity to local chemical species.

\subsubsection{Biodegradable polymers}

If the implanted material is only temporary but requires reintegration and remodeling, a degradable material with nontoxic byproducts is often a good choice for materials selection. One of the most popular clinically used polymers for this type of application is poly-L-lactic acid (PLLA). PLLA is a biodegradable, crystalline polymer with excellent mechanical properties for applications such as sutures. The properties of the PLLA can be tuned such that it can take as long as 2 to 6 years before it is completely reabsorbed into the body, which is much longer than most biodegradable polymers used in medicine ${ }^{46-47}$. Other biodegradable polymers have faster degradation times such as polyglycolide (PGA), which has been used as a copolymer with PLLA to form poly(lactide-co-glycolide) (PLGA), which has adjustable degradation times based on the ratio of PGA to PLLA ${ }^{48}$.

\subsubsection{Hydrogels}

Hydrogels are another class of polymer with many uses in medicine. They are described as synthetic, hydrophilic polymers that swell when exposed to water ${ }^{49}$. This property allows hydrogels to be applied to areas of controlled drug release. As water enters the polymer matrix, polymeric chains become more loosely bound, allowing the release of any drug the hydrogel could be containing ${ }^{50}$. Hydrogels most commonly release their desired drugs from a physical change $(\mathrm{pH}$, temperature, hydrophobicity) rather than through a typical chemical reaction where covalent bonds are cleaved. Along with drug release, the structural aspect of hydrogels is also an important parameter. Polyethylene glycol (PEG) hydrogels specifically have been used as tissue repair scaffolds. PEG has the ability to change its material properties simply by adjusting parameters such as crosslinking density and molecular weight to alter the material and chemical properties 
of the hydrogel ${ }^{51}$. Chemical crosslinking needs to be taken carefully into consideration as some crosslinkers can react with the drug trying to be administered, causing the formation of unwanted, possibly toxic products, which will eventually lead to a large immune response from the host ${ }^{52}$.

\subsubsection{Hydrophobic polymers}

Unlike the polymers used in hydrogels, hydrophobic polymers have no water uptake and have unique properties when in contact with cells and tissue. The most popular class of hydrophobic polymers used in biomedical applications are silicone rubber based materials. Polydimethylsiloxane (PDMS) based silicone rubber have been shown to have excellent biocompatibility, low toxicity, stability in a physiological environment, and are chemically inactive ${ }^{53-56}$. Using a hydrophobic material like PDMS is useful for long lasting implantable applications that resist the cellular infiltration and remodeling seen in the biodegradable and hydrophilic materials. Like any long term implanted material, PDMS and other hydrophobic polymers still see issues of chronic inflammation, showing that another modification to these materials is needed to overcome the body's response to foreign materials.

\subsection{Background of Current Nitric Oxide Donating Materials}

\subsubsection{Nitric oxide chemistry}

In order to be able to mimic the body's range of production of NO at different physiological locations, many NO donating materials have been synthesized. Since NO is a highly reactive free radical molecule, strategies of covalently linking it to materials to stabilize and control it were developed. The two most widely used synthetic NO donors are based on the chemistry of S-nitrosothiols (RSNOs) and N-diazeniumdiolates (NONOates). Choosing which type of NO donor is important as each has their own set of release mechanisms, stability under physiological conditions, and covalent linking chemistry. Having a wide variety of controllable parameters gives a large amount of 
flexibility when deciding where and how you want NO to be delivered. This localized and highly controlled delivery is important to harness the therapeutic potential of $\mathrm{NO}$ in part because NO has a half-life ranging from seconds to minutes depending on the physiological environment it's exposed to ${ }^{57-59}$.

\subsubsection{S-Nitrosothiols}

For RSNO modified materials, there is a degree of control with how NO is administered to an area as can be highly stable under in vivo conditions if appropriate precautions are taken. The main mechanisms for NO to be cleaved from an RSNO system are by light and metal ions ${ }^{60-61}$. Photolytic cleavage of the sulfur-nitrogen bond occurs at the wavelengths of $340 \mathrm{~nm}$ and $545 \mathrm{~nm}^{62}$. Knowing this chemical property gives the possibility of using RSNO based materials as a controlled therapeutic for the administration of NO. RSNO's are among the safest and most biocompatible NO donors to use within materials as they are produced endogenously in the form Snitrosoglutathione (GSNO). This formation occurs by the reaction of glutathione (GSH) with $\mathrm{N}_{2} \mathrm{O}_{3}$ to form GSNO. A key RSNO that is used for NO delivery from polymeric biomaterials is S-nitroso-N-acetyl-D-penicillamine (SNAP) (Figure 1.6). Both SNAP and GSNO have been shown to have positive effects to vasculature in respects with vasodilation and preventing platelet adhesion ${ }^{63}$. SNAP is more reactive with ions such as iron and copper to trigger NO release than GSNO. The reaction of copper with RSNOs has been thoroughly investigated as one of the key metal triggers. Copper (II) ions $\left(\mathrm{Cu}^{2+}\right)$ specifically interact with RSNOs by first being reduced to $\mathrm{Cu}^{+}$by thiol anions present. $\mathrm{Cu}^{+}$is then able to cleave the sulfur-nitrogen bond while being regenerated back to $\mathrm{Cu}^{2+}$ The cleaved sulfur complexes can then form disulfide bonds as the reaction cycle continues ${ }^{64}$. 
<smiles>NC(CCC(=O)NC(CSN=O)C(=O)NCC(=O)O)C(=O)O</smiles>

GSNO<smiles>CC(=O)NC(C(=O)O)C(C)(C)SN=O</smiles>

SNAP

Figure 1.6. Structure of S-nitrosoglutathione (GSNO) and S-nitroso-N-acetyl-Dpenicillamine (SNAP). GSNO is an endogenous S-nitrosothiol commonly found in the body while SNAP is used in chemical synthesis to artificially add nitric oxide donating groups to synthetic materials.

\subsubsection{N-diazeniumdiolates}

Diazeniumdiolates, also known as NONOates, are a class of zwitterionic compound that are used in the NO donating research field with different chemical properties compared to RSNO's. The first investigation of using NONOates as a potential drug administering molecule was done by Dr. Larry K. Keefer at the National Cancer Institute. The release kinetics and dissociation properties of NONOates were investigated and proved to have useful characteristics under physiological $\mathrm{pH}$ and temperature ${ }^{65}$. Two molecules of NO per NONOate molecule are able to be produced and these NONOates can have a halflife of up to 20 hours under these conditions, depending on the surrounding functional groups ${ }^{66}$. The release mechanism of NO from these molecules is either via proton mediated decomposition or thermal degradation ${ }^{66}$. This opens up a wide range of applications for areas that require short and large high amounts of localized NO as the donor can be covalently bound to larger molecules or polymers. However, these molecules some of the controllability and in vivo longevity compared to some RSNOs. Dimethylhexanediamine diazeniumdiolates $\left(\mathrm{DMHD} / \mathrm{N}_{2} \mathrm{O}_{2}\right)$ is a popular NO donating material for these applications, and has been blended in with multiple polymer matrices to demonstrate its ability to release a large amount of NO over a short duration (Figure 1.7). 


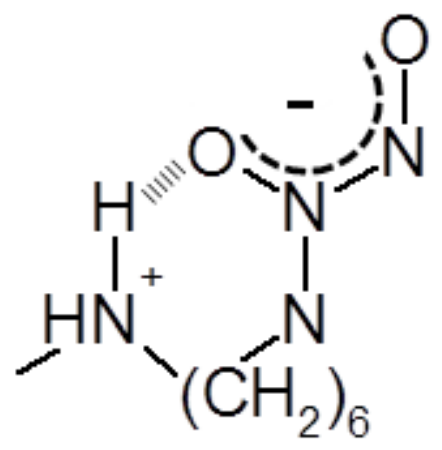

Figure 1.7. Structure of dimethylhexanediamine diazeniumdiolates (DMHD/ $\left.\mathrm{N}_{2} \mathrm{O}_{2}\right)$. This zwitterionic NO donating structure allows for the release of two moles of NO per molecule when placed under physiological conditions. The release is passive and very rapid - making it an excellent choice of NO donor for short term passive release.

\subsection{Applications of Nitric Oxide Donating Materials}

\subsubsection{Concerns for nitric oxide concentration}

Determining the proper amount of NO to be administered to an area in the body when developing synthetic NO releasing materials is critical. Depending on the level and duration of NO generated, it can be used to mediate cell proliferation and protect them, or it can be used to trigger cell apoptosis. Concentration of NO determines how far NO is capable of diffusing and consequently what type of signaling molecules it interacts with. Table 1.1 gives an overview of the range of concentrations NO and its corresponding cellular function. Although the concentrations are given in molar concentrations, in a true physiological environment, NO exists mainly as a flux being emitted from a cellular source. The concentrations stated are estimates based on the NO dissolved in solution around the environment. 
Table 1.1. Effect of NO when administered at varying concentrations to certain cell types. The effects of NO have a large variety based on the concentration being administered, along with where in the body the release is occurring. When developing synthetic materials that are able to release NO, it is important to be aware of the concentrations being administered to achieve the desired outcome.

\begin{tabular}{|c|c|c|}
\hline $\begin{array}{l}\text { NO } \\
\text { concentration }\end{array}$ & Physiological Effect & References \\
\hline $1-30 \mathrm{nM}$ & $\begin{array}{l}\text { - } \begin{array}{l}\text { Endothelial cell release of NO (eNOS } \\
\text { mechanism) }\end{array} \\
\text { - Leads to smooth muscle relaxation } \\
\text { through conversion of sGC to cGMP } \\
\text { - Maintains vascular tone } \\
\text { - Cell proliferative effects }\end{array}$ & 67 \\
\hline $30-60 \mathrm{nM}$ & $\begin{array}{ll}\text { - } & \text { Levels emitted from tumor cells } \\
\text { - } & \text { Tumor proliferation } \\
\text { - } & \text { Antiapoptotic }\end{array}$ & $688-69$ \\
\hline$\sim 100 \mathrm{nM}$ & $\begin{array}{l}\text { - Hypoxic inducible factor } 1 \alpha(\mathrm{HIF}-1 \alpha) \\
\text { accumulation } \\
\text { - VEGF accumulation }\end{array}$ & $69-70$ \\
\hline$\sim 400 \mathrm{nM}$ & $\begin{array}{l}\text { - Upregulation of p53 - caused by damage } \\
\text { to DNA } \\
\text { - Antipathogen and tumorcidal } \\
\text { - Macrophage release of NO (iNOS } \\
\text { mechanism) }\end{array}$ & 70 \\
\hline
\end{tabular}




\begin{tabular}{|l|l|l|}
\hline$>1 \mu \mathrm{M}$ & $\bullet$ & Nitrosative stress - Protein \\
& nitrosation/nitration & 71 \\
$\bullet$ & Inhibition of DNA repair and \\
& mitochondrial respiration \\
& Leads to full cell cycle arrest & \\
& & \\
\hline
\end{tabular}

\subsubsection{Improving biocompatibility of foreign devices}

Controlled NO releasing materials that are capable of delivering the appropriate level of $\mathrm{NO}$ at the appropriate time can be used to improve biocompatibility of blood and tissue contacting devices, creating safer and more effective implanted devices such as intravascular and subcutaneous sensors, and has the broader potential to improve performance of virtually all implanted biomedical devices. A specific example would be synthesizing a material to copy the NO release seen from healthy vasculature, which is estimated to be approximately $0.5-4 \times 10^{-10} \mathrm{~mol} \mathrm{~cm}^{-2} \mathrm{~min}^{-1}$ depending on the specific location within the circulatory system ${ }^{72}$. Various polymeric materials have been fabricated that release $\mathrm{NO}$ at this level and have shown much success in inhibiting platelet adhesion and activation to foreign blood contacting materials and lowering the chronic inflammatory response of subcutaneous tissue contacting materials ${ }^{73-77}$. Experimental setups have been design using an extracorporeal circuit (ECC) for in vivo experiments on rabbits to prove NO releasing material's effect on platelet adhesion to foreign materials ${ }^{78}$.

\subsubsection{Interaction with macrophages and mast cells}

NO's effect on macrophages is still being investigated as various levels of NO can influence them in different manners. When trying to apply an NO donating material to ward off macrophage attachment and recruitment, NO flux must be carefully considered. Large amounts of NO being delivered to a site where macrophages are 
present can prevent the production of recruitment cytokines, while small amounts can actually influence a higher production of these inflammatory inducing cytokines ${ }^{79}$. Lung macrophages in culture have been treated with NO from SNAP at concentrations ranging from millimolar to micromolar, and were shown to have a decrease in proinflammatory cytokines ${ }^{80}$. There is also the risk of trying to utilize a high, uncontrolled dose of NO to prevent this signaling as a bolus of NO will cause cell death and tissue necrosis.

Mast cells are another large contributor to the immediate inflammatory response to a biomaterial. The primary function of mast cells is the release of histamine to cause vasopermeation between vascular endothelial cells to allow the migration of leukocytes to the injury site. NO has been previously shown to inhibit the histamine release from activated mast cells to mediate this inflammatory response ${ }^{81-82}$. Specifically, NO's interaction with interferon gamma (IFN- $\gamma$ ) was investigated. IFN- $\gamma$ induces NO production from nearby cell populations, which then directly inhibits mast cell degranulation ${ }^{83-84}$. Experiments were performed by inhibiting NO production through NOS by administering a competitive inhibitor in a culture of tissue type mast cells containing IFN- $\gamma$. Once the NO production was allowed, there was a drastic decrease in serotonin release from mast cells, signifying NO's effect to prevent degranulation. Degranulated mast cells also release cytokines like IL-4 and IL-13, which play large roles in determining how harsh and long the foreign body reaction will persist ${ }^{85}$. Using NO to prevent this degranulation process and release of cytokines could be a large benefit for slowing chronic inflammation.

\subsubsection{Role in vascular diseases}

Applying NO release as a treatment for vascular disorders has also been studied. After balloon angioplasty treatment, there is damage to the endothelium which eventually leads to myointimal hyperplasia caused from the migration of smooth muscle cells to the lumen of the vasculature and eventually proliferation ${ }^{86}$. A cascade of events then occur which causes the occlusion to worsen - smooth muscle cells change phenotype 
and start to produce ECM, the adhesion of platelets to the newly formed ECM, and eventually the recruitment of more inflammatory cells to the region worsen ${ }^{87}$. NO at these damaged vascular sites can prevent all of these negative effects caused as vascular smooth muscle cell proliferation is inhibited by NO as well as platelet activation as mentioned previously ${ }^{88}$. Other disorders that are due to a reduction in NO activity within vasculature are hypercholesterolemia and atherosclerosis ${ }^{89-90}$. Oxidized lowdensity lipoproteins (LDLs) are the leading cause for hypercholesterolemia as it reacts and inactivates endogenous $\mathrm{NO}^{91-92}$. It also causes the generation of superoxide anions from the endothelium and migrating macrophages, creating an environment of high oxidative stress ${ }^{41}$. This highly oxidative area scours and reacts with NO that would otherwise be promoting healthy vascular function. For coronary artery diseases, saphenous vein grafts eventually undergo atherosclerosis and vessel occlusion within the first 10 years due to the migration and proliferation of smooth muscles cells ${ }^{93}$. nNOS gene transfer to these saphenous vein graft sites demonstrated the ability of NO to a large reduction in these negative effects, prolonging the life of these grafts ${ }^{94}$.

\subsubsection{Cancer therapeutic applications}

Another application for highly controlled NO releasing materials is in drug delivery to tumors. NO in appropriate doses is known to induce apoptosis in cells and could function as an effective local cancer treatment ${ }^{95}$. NO in an oxidative environment reacts to create peroxynitrites, which is seen specifically in activated macrophages. Tumors have been shown to have increased levels of superoxides and other ROSs, giving NO the ability to be a potent cancer therapeutic when applied correctly ${ }^{96-69}$. If exposed to the right level of NO, the cancerous region would be exposed to localized oxidative byproducts, damaging the tumor, while other nearby areas could be left relatively unharmed as the ROS level would be much lower. This level of NO must be administered carefully as certain fluxes of NO can also increase tumor angiogenesis ${ }^{97}$. NO has been shown to promote vascularity and increase the production of vascular endothelial growth factor (VEGF) in endothelial cells ${ }^{98}$. 


\subsubsection{Reendothelialization of vessels}

In other environments, the increase in vascularity from endothelial cells could be seen as a positive effect. Decellularized vascular implants or biodegradable materials that need cellular infiltration and revascularization would benefit greatly from the increase in production of VEGF from endothelial cells, allowing the opportunity of NO donating materials being able to revascularize vessel grafts at a much quicker rate while still preventing unwanted inflammation at the implantation site ${ }^{74,98-99}$. In unmodified vessel grafts, smooth muscle cells are the primary cell type that infiltrate and proliferate, causing occlusion of the vessel. NO would be able to promote the infiltration of endothelial cells in its place from the neighboring endothelial cells. Grafts incorporating NO have been done previously and shown to prevent platelet adhesion and recruitment to these sites that would otherwise perpetuate this inflammatory response to eventually lead to graft failure ${ }^{100}$.

\subsection{Detection Methods of Nitric Oxide}

\subsubsection{Griess assay}

The most important part of analyzing any NO releasing material is the methods used to determine and quantify the release into the surrounding environment. Since NO is a free radical gas, accurate detection becomes complex due to its high reactivity with the surrounding environment. A popular method to determine NO production is to measure the amount of nitrites in solution is through the Griess assay, which was formulated by Peter Griess in $1879^{101}$ (Figure 1.8). This method can be used to quantify the amount of $\mathrm{NO}$ in solution by indirectly measuring the oxidation products that are formed when reacted with oxygen. These oxidation products can then react with sulfanilamide and $\mathrm{N}$ 1-naphthylethylenediamine to form a fluorescent azo dye. Using a plate reader, the dye formed can be detected using a $550 \mathrm{~nm}$ filter which then quantifies the amount of NO being converted to nitrites. The absorbance found can then be compared to a calibration 
curve of nitrite values to give the amount of NO released into solution. This method can give false results though as $\mathrm{NO}$ can readily react with oxygen in solution to form nitrate molecules, which would give lower amounts of NO than what is being measured ${ }^{102}$.

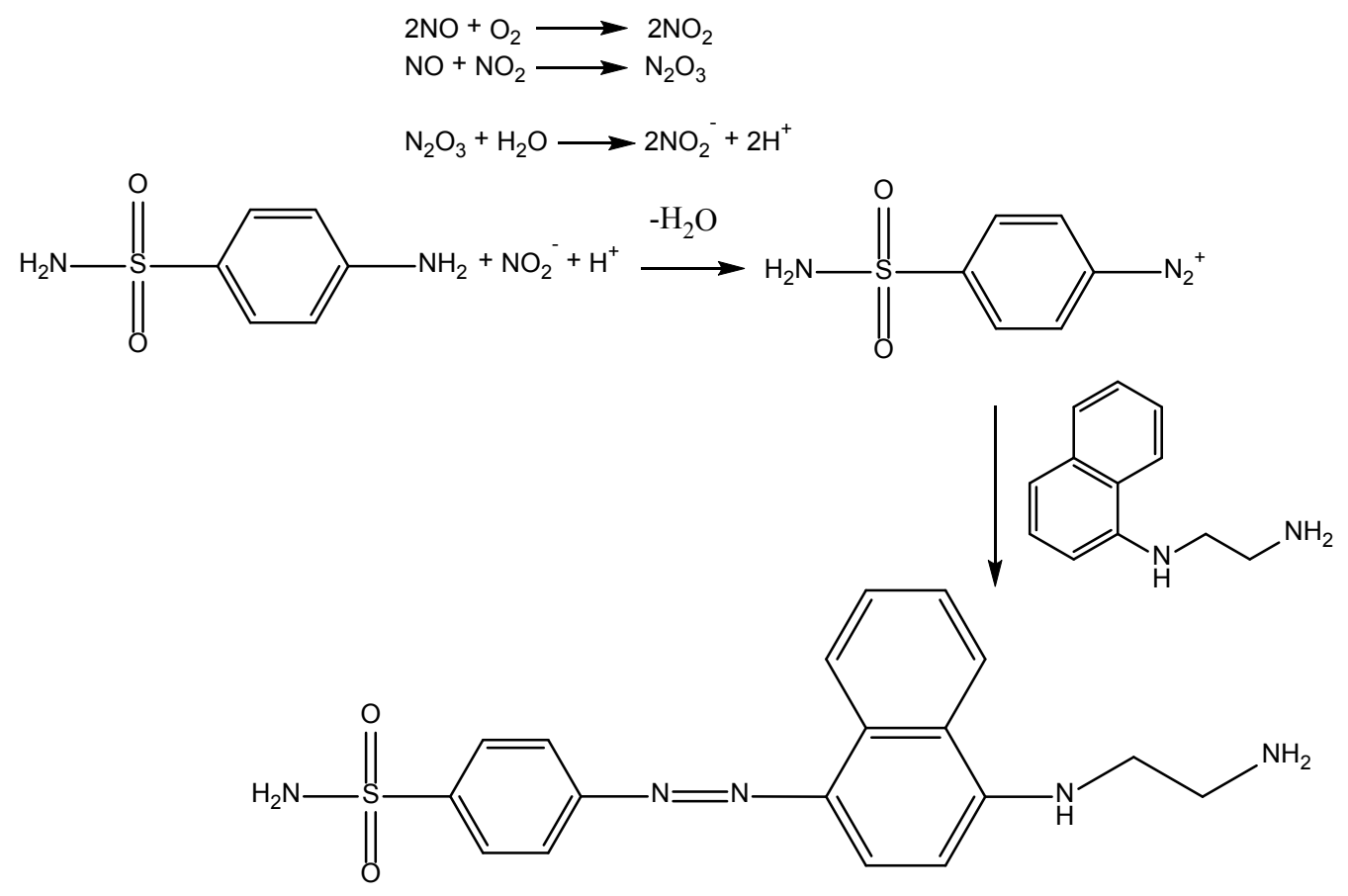

Figure 1.8. Chemical detection of NO through the Griess reaction.

$\mathrm{NO}$ is first converted into a nitrite product, where it then interacts with sulfanilamide and $\mathrm{N}$-1-napthylethylenediamine to form a fluorescent detectable dye which is detectable at $540 \mathrm{~nm}$. This can then be used to quantify the amount of NO that was released within a solution.

\subsubsection{Electrochemical}

Electrochemical detection of $\mathrm{NO}$ is another method for quantifying $\mathrm{NO}$ release from a material (Figure 1.9). There are two strategies for electrochemically identifying $\mathrm{NO}-$ electrooxidation and electroreduction through the use of $\mathrm{Ag} / \mathrm{AgCl}$ electrodes. Electrooxidation reduces $\mathrm{NO}$ to generate $\mathrm{N}_{2} \mathrm{O}_{2}{ }^{2-}$ through a single step, two electron reaction, while electroreduction of $\mathrm{NO}$ is completed through a three step pathway where $\mathrm{NO}$ is oxidized to form nitric acid, and then is further oxidized in water to form $\mathrm{NO}_{3}{ }^{-}$ ${ }^{103-104}$. Electrochemical electrodes have been shown to be able to detect NO 
continuously in a biological environment that is generating NO in real time ${ }^{105}$. This would give insight to $\mathrm{NO}$ generation from cells in vivo as electrodes can be constructed small enough to monitor vasculature and organs. The downside for this method is that its detection limit of $1 \mu \mathrm{M}$ is much less sensitive when compared to the chemiluminescent and Griess assays ${ }^{106}$. There could also be interference based on how far the electrode is from the site that is generating NO. In an in vivo environment, NO has a very short half-life and might not even come into contact with the electrode if it is not placed properly ${ }^{107}$.

Electrooxidation

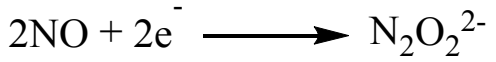

Electroreduction

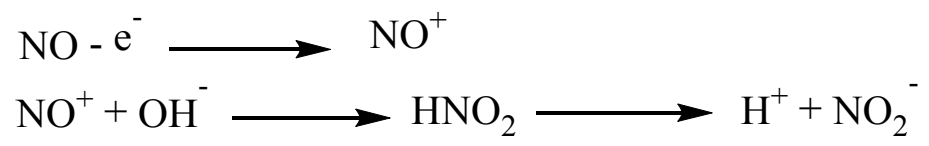

Figure 1.9. Chemical mechanisms for the electrochemical detection of NO. Through electrooxidation, $\mathrm{NO}$ undergoes a two electron reduction to form $\mathrm{N}_{2} \mathrm{O}_{2}{ }^{2-}$.

Electroreduction goes through a multistep oxidation path to form $\mathrm{NO}_{2}^{-}$from $\mathrm{NO}$. Concentration of NO is then measured by referring the current recorded to a known calibration standard.

\subsubsection{Chemiluminescence}

The most accurate method to directly measure NO coming from a either a NO releasing sample or cells in media is through chemiluminescence (Figure 1.10). Using a system purged with nitrogen, there are no reactive side products to interfere with NO measurement as seen with other NO detection assays. It also gives a detailed profile of NO being released over a period of time. This allows the calculation of NO fluxes from cells and materials, which is an important parameter to keep in mind when calibrating NO releasing biomaterials to the physiological levels of release seen in the body. For this detection method, NO reacts with ozone to produce oxygen and nitrogen dioxide in 
an excited state. During the relaxation of nitrogen dioxide, a photon is emitted which can be measured ${ }^{108}$.

$$
\begin{aligned}
& \mathrm{NO}+\mathrm{O}_{3} \longrightarrow \mathrm{NO}_{2}{ }^{*}+\mathrm{O}_{2} \\
& \mathrm{NO}_{2}{ }^{*} \longrightarrow \mathrm{NO}_{2}+\mathrm{hv}
\end{aligned}
$$

Figure 1.10. Mechanism for the detection of NO through chemiluminescence. NO is initially reacted with ozone to form a nitrogen dioxide in the excited state. As this molecule relaxes, it emits a photon which can then be measured and quantified.

\subsection{Statement of Purpose}

Knowing all of the beneficial effects and applications of NO is crucial when developing a unique NO donating material. Chapter 2 describes an NO donating PVC material which utilizes covalent bound RSNOs. By covalently attaching the NO donor to the backbone of the polymer, it allows a uniform NO flux across the polymer area when triggered by light for controlled release. This characteristic gives it beneficial uses for in vitro NO delivery models. Chapter 3 utilizes dendrimer chemistry to develop a highly branched polymeric structure capable of high NO storage. Most NO donors that passively or actively release NO do not last for long periods of time. By synthesizing a hyperbranched molecule with high NO capacity, a polymer can be loaded with a tremendous amount of NO capable of delivering NO longer than most other NO donors. Chapter 4 describes how subcutaneous inflammation can be controlled using the two materials previously described. The foreign body response to subcutaneously implanted devices dramatically reduces its longevity. By utilizing these beneficial effects of NO described, the developed materials were used to prevent the harsh inflammatory responses normally observed. This can then be applied to any future subcutaneously implanted devices to increase its implantation life, reducing the amount of interventions required to constantly replace sensors needed to record specific analytes. The preliminary data in chapter 5 delves into the potential effects NO can have on viruses. The mechanisms by which macrophages use NO to kill foreign bacteria is hypothesized 
to destroy viruses in a similar manner, but the exact dosage is still unknown. The groundwork for that chapter describes an accurate method of delivering NO to viruses suspended in solution to find this dosage level.

\subsection{References}

1. Katsuki, S.; Arnold, W.; Mittal, C.; Murad, F., Stimulation of guanylate cyclase by sodium nitroprusside, nitroglycerin and nitric oxide in various tissue preparations and comparison to the effects of sodium azide and hydroxylamine. Journal of cyclic nucleotide research 1977, 3 (1), 23-35.

2. Marsh, N.; Marsh, A., A Short History Of Nitroglycerine And Nitric Oxide In Pharmacology And Physiology. Clinical and Experimental Pharmacology and Physiology 2000, 27 (4), 313-319.

3. Furchgott, R. F., Role of endothelium in responses of vascular smooth muscle. Circulation research 1983, 53 (5), 557-573.

4. Ignarro, L. J.; Buga, G. M.; Wood, K. S.; Byrns, R. E.; Chaudhuri, G., Endothelium-derived relaxing factor produced and released from artery and vein is nitric oxide. Proceedings of the National Academy of Sciences 1987, 84 (24), 9265-9269.

5. Palmer, R. M.; Ferrige, A.; Moncada, S., Nitric oxide release accounts for the biological activity of endothelium-derived relaxing factor. 1987.

6. Knowles, R. G.; Moncada, S., Nitric oxide synthases in mammals. Biochemical Journal 1994, 298 (Pt 2), 249.

7. Marletta, M. A., Nitric oxide synthase: Aspects concerning structure and catalysis. Cell 1994, 78 (6), 927-930.

8. Palmer, R. M.; Ashton, D.; Moncada, S., Vascular endothelial cells synthesize nitric oxide from L-arginine. Nature 1988, 333 (6174), 664-666.

9. Marsden, P. A.; Schappert, K. T.; Chen, H. S.; Flowers, M.; Sundell, C. L.; Wilcox, J. N.; Lamas, S.; Michel, T., Molecular cloning and characterization of human endothelial nitric oxide synthase. FEBS letters 1992, 307 (3), 287-293. 
10. Corson, M. A.; James, N. L.; Latta, S. E.; Nerem, R. M.; Berk, B. C.; Harrison, D. G., Phosphorylation of Endothelial Nitric Oxide Synthase in Response to Fluid Shear Stress. Circulation Research 1996, 79 (5), 984-991.

11. Yamashita, T.; Kawashima, S.; Ohashi, Y.; Ozaki, M.; Rikitake, Y.; Inoue, N.; Hirata, K. i.; Akita, H.; Yokoyama, M., Mechanisms of Reduced Nitric Oxide/cGMP-Mediated Vasorelaxation in Transgenic Mice Overexpressing Endothelial Nitric Oxide Synthase. Hypertension 2000, 36 (1), 97-102.

12. Radomski, M.; Palmer, R.; Moncada, S., Endogenous nitric oxide inhibits human platelet adhesion to vascular endothelium. The Lancet 1987, 330 (8567), 1057-1058.

13. Radomski, M.; Palmer, R.; Moncada, S., An L-arginine/nitric oxide pathway present in human platelets regulates aggregation. Proceedings of the National Academy of Sciences 1990, 87 (13), 5193-5197.

14. Bredt, D. S.; Hwang, P. M.; Snyder, S. H., Localization of nitric oxide synthase indicating a neural role for nitric oxide. Nature 1990, 347 (6295), 768-70.

15. Prast, H.; Philippu, A., Nitric oxide as modulator of neuronal function. Progress in neurobiology 2001, 64 (1), 51-68.

16. Lee, S. J.; Stull, J. T., Calmodulin-dependent Regulation of Inducible and Neuronal Nitric-oxide Synthase. Journal of Biological Chemistry 1998, 273 (42), 27430-27437.

17. Xie, Q.; Cho, H.; Calaycay, J.; Mumford, R.; Swiderek, K.; Lee, T.; Ding, A.; Troso, T.; Nathan, C., Cloning and characterization of inducible nitric oxide synthase from mouse macrophages. Science 1992, 256 (5054), 225-228.

18. Ischiropoulos, H.; Zhu, L.; Beckman, J. S., Peroxynitrite formation from macrophage-derived nitric oxide. Archives of Biochemistry and Biophysics 1992, 298 (2), 446-451.

19. Frost, M. C.; Reynolds, M. M.; Meyerhoff, M. E., Polymers incorporating nitric oxide releasing/generating substances for improved biocompatibility of bloodcontacting medical devices. Biomaterials 2005, 26 (14), 1685-93. 
20. Anderson, J. M.; Rodriguez, A.; Chang, D. T. In Foreign body reaction to biomaterials, Seminars in immunology, Elsevier: 2008; pp 86-100.

21. Park, H.; Park, K., Biocompatibility issues of implantable drug delivery systems. Pharmaceutical research 1996, 13 (12), 1770-1776.

22. Gratchev, A.; Guillot, P.; Hakiy, N.; Politz, O.; Orfanos, C.; Schledzewski, K.; Goerdt, S., Alternatively Activated Macrophages Differentially Express Fibronectin and Its Splice Variants and the Extracellular Matrix Protein $\beta I G-H 3$. Scandinavian journal of immunology 2001, 53 (4), 386-392.

23. Song, E.; Ouyang, N.; Hörbelt, M.; Antus, B.; Wang, M.; Exton, M. S., Influence of alternatively and classically activated macrophages on fibrogenic activities of human fibroblasts. Cellular immunology 2000, 204 (1), 19-28.

24. Sternlicht, M. D.; Werb, Z., How matrix metalloproteinases regulate cell behavior. Annual review of cell and developmental biology 2001, 17, 463.

25. Marchant, R.; Hiltner, A.; Hamlin, C.; Rabinovitch, A.; Slobodkin, R.; Anderson, J. M., In vivo biocompatibility studies. I. The cage implant system and a biodegradable hydrogel. Journal of biomedical materials research 1983, 17 (2), 301-325.

26. Anderson, J. M., Biological responses to materials. Annual Review of Materials Research 2001, 31 (1), 81-110.

27. Cotran, R. S.; Kumar, V.; Collins, T.; Robbins, S. L., Robbins pathologic basis of disease. 1999.

28. Broughton 2nd, G.; Janis, J. E.; Attinger, C. E., The basic science of wound healing. Plastic and reconstructive surgery 2006, 117 (7 Suppl), 12S-34S.

29. DeFife, K. M.; Jenney, C. R.; McNally, A. K.; Colton, E.; Anderson, J. M., Interleukin-13 induces human monocyte/macrophage fusion and macrophage mannose receptor expression. The Journal of Immunology 1997, 158 (7), 33853390.

30. Kao, W. J.; McNally, A. K.; Hiltner, A.; Anderson, J. M., Role for interleukin-4 in foreign-body giant cell formation on a poly (etherurethane urea) in vivo. Journal of biomedical materials research 1995, 29 (10), 1267-1275. 
31. McNally, A. K.; Anderson, J. M., Interleukin-4 induces foreign body giant cells from human monocytes/macrophages. Differential lymphokine regulation of macrophage fusion leads to morphological variants of multinucleated giant cells. The American journal of pathology 1995, 147 (5), 1487.

32. Jones, J. A.; Dadsetan, M.; Collier, T. O.; Ebert, M.; Stokes, K. S.; Ward, R. S.; Hiltner, P. A.; Anderson, J. M., Macrophage behavior on surface-modified polyurethanes. Journal of Biomaterials Science, Polymer Edition 2004, 15 (5), 567-584.

33. Henson, P. M., The immunologic release of constituents from neutrophil leukocytes II. Mechanisms of release during phagocytosis, and adherence to nonphagocytosable surfaces. The Journal of Immunology 1971, 107 (6), 15471557.

34. Henson, P. M., The immunologic release of constituents from neutrophil leukocytes I. The role of antibody and complement on nonphagocytosable surfaces or phagocytosable particles. The Journal of Immunology 1971, 107 (6), 1535-1546.

35. Galván-Peña, S.; O’Neill, L. A., Metabolic reprograming in macrophage polarization. Frontiers in immunology 2014, 5 .

36. Novak, M. L.; Koh, T. J., Macrophage phenotypes during tissue repair. Journal of leukocyte biology 2013, 93 (6), 875-881.

37. Odegaard, J. I.; Chawla, A., Alternative macrophage activation and metabolism. Annual review of pathology 2011, 6, 275.

38. Murray, P. J.; Wynn, T. A., Protective and pathogenic functions of macrophage subsets. Nature reviews immunology 2011, 11 (11), 723-737.

39. Munder, M.; Eichmann, K.; Morán, J. M.; Centeno, F.; Soler, G.; Modolell, M., Th1/Th2-regulated expression of arginase isoforms in murine macrophages and dendritic cells. The Journal of Immunology 1999, 163 (7), 3771-3777.

40. Moyer, A. L.; Wagner, K. R., Regeneration versus fibrosis in skeletal muscle. Current opinion in rheumatology 2011, 23 (6), 568-573. 
41. Ohara, Y.; Peterson, T. E.; Harrison, D. G., Hypercholesterolemia increases endothelial superoxide anion production. Journal of Clinical Investigation 1993, $91(6), 2546$.

42. Angelova, N.; Hunkeler, D., Rationalizing the design of polymeric biomaterials. Trends in biotechnology 1999, 17 (10), 409-421.

43. Buján, J.; García-Honduvilla, N.; Contreras, L.; Gimeno, M. J.; Escudero, C.; Bellón, J. M.; San-Román, J., Coating PTFE vascular prostheses with a fibroblastic matrix improves cell retention when subjected to blood flow. Journal of biomedical materials research 1998, 39 (1), 32-39.

44. Yoda, R., Elastomers for biomedical applications. Journal of Biomaterials Science, Polymer Edition 1998, 9 (6), 561-626.

45. Haas, A., The phagosome: compartment with a license to kill. Traffic 2007, 8 (4), 311-330.

46. Middleton, J. C.; Tipton, A. J., Synthetic biodegradable polymers as orthopedic devices. Biomaterials 2000, 21 (23), 2335-2346.

47. Bergsma, J.; Rozema, F.; Bos, R.; Boering, G.; De Bruijn, W.; Pennings, A., In vivo degradation and biocompatibility study of in vitro pre-degraded aspolymerized polylactide particles. Biomaterials 1995, 16 (4), 267-274.

48. Nair, L. S.; Laurencin, C. T., Biodegradable polymers as biomaterials. Progress in polymer science 2007, 32 (8), 762-798.

49. Peppas, N. A., Hydrogels in medicine and pharmacy. CRC press Boca Raton, FL: 1987; Vol. 3.

50. Gayet, J.-C.; Fortier, G., High water content BSA-PEG hydrogel for controlled release device: Evaluation of the drug release properties. Journal of controlled release 1996, 38 (2), 177-184.

51. Lin, C.-C.; Anseth, K. S., PEG hydrogels for the controlled release of biomolecules in regenerative medicine. Pharmaceutical research 2009, 26 (3), 631-643. 
52. Soyez, H.; Schacht, E.; Jelinkova, M.; Rihova, B., Biological evaluation of mitomycin C bound to a biodegradable polymeric carrier. Journal of controlled release 1997, 47 (1), 71-80.

53. Polmanteer, K. E., Silicone rubber, its development and technological progress. Rubber chemistry and technology 1988, 61 (3), 470-502.

54. Quinn, K. J.; Courtney, J. M., Silicones as biomaterials. British polymer journal 1988, 20 (1), 25-32.

55. Peterson, S. L.; McDonald, A.; Gourley, P. L.; Sasaki, D. Y., Poly (dimethylsiloxane) thin films as biocompatible coatings for microfluidic devices: cell culture and flow studies with glial cells. Journal of Biomedical Materials Research Part A 2005, 72 (1), 10-18.

56. Abbasi, F.; Mirzadeh, H.; Katbab, A. A., Modification of polysiloxane polymers for biomedical applications: a review. Polymer International 2001, 50 (12), 1279-1287.

57. Butler, A. R.; Flitney, F. W.; Williams, D. L. H., NO, nitrosonium ions, nitroxide ions, nitrosothiols and iron-nitrosyls in biology: a chemist's perspective. Trends in pharmacological sciences 1995, 16 (1), 18-22.

58. Wink, D. A.; Hanbauer, I.; Grisham, M. B.; Laval, F.; Nims, R. W.; Laval, J.; Cook, J.; Pacelli, R.; Liebmann, J.; Krishna, M., Chemical biology of nitric oxide: regulation and protective and toxic mechanisms. Current topics in cellular regulation 1995, 34, 159-187.

59. Stamler, J. S., Redox signaling: nitrosylation and related target interactions of nitric oxide. Cell 1994, 78 (6), 931-936.

60. Williams, D. L. H., S-Nitrosothiols and role of metal ions in decomposition to nitric oxide. 1996, 268, 299-308.

61. Williams, D. L. H., The chemistry of S-nitrosothiols. Accounts of chemical research 1999, 32 (10), 869-876.

62. Sexton, D. J.; Muruganandam, A.; McKenney, D. J.; Mutus, B., Visible light photochemical release of nitric oxide from S-nitrosoglutathione: Potential 
photochemotherapeutic applications. Photochemistry and photobiology 1994, 59 (4), 463-467.

63. Askew, S. C.; Butler, A. R.; Flitney, F. W.; Kemp, G. D.; Megson, I. L., Chemical mechanisms underlying the vasodilator and platelet anti-aggregating properties of S-nitroso-N-acetyl-dl-penicillamine and S-nitrosoglutathione. Bioorganic \& Medicinal Chemistry 1995, 3 (1), 1-9.

64. Al-Sa'doni, H.; Ferro, A., S-Nitrosothiols: a class of nitric oxide-donor drugs. Clinical science 2000, 98 (5), 507-520.

65. Davies, K. M.; Wink, D. A.; Saavedra, J. E.; Keefer, L. K., Chemistry of the diazeniumdiolates. 2. Kinetics and mechanism of dissociation to nitric oxide in aqueous solution. Journal of the American Chemical Society 2001, 123 (23), 5473-5481.

66. Fitzhugh, A. L.; Keefer, L. K., Diazeniumdiolates:: Pro-and antioxidant applications of the "NONOates". Free Radical Biology and Medicine 2000, 28 (10), 1463-1469.

67. Thomas, D. D.; Ridnour, L. A.; Espey, M. G.; Donzelli, S.; Ambs, S.; Hussain, S. P.; Harris, C. C.; DeGraff, W.; Roberts, D. D.; Mitchell, J. B., Superoxide fluxes limit nitric oxide-induced signaling. Journal of Biological Chemistry 2006, 281 (36), 25984-25993.

68. Pervin, S.; Singh, R.; Hernandez, E.; Wu, G.; Chaudhuri, G., Nitric oxide in physiologic concentrations targets the translational machinery to increase the proliferation of human breast cancer cells: involvement of mammalian target of rapamycin/eIF4E pathway. Cancer Research 2007, 67 (1), 289-299.

69. Ridnour, L. A.; Thomas, D. D.; Switzer, C.; Flores-Santana, W.; Isenberg, J. S.; Ambs, S.; Roberts, D. D.; Wink, D. A., Molecular mechanisms for discrete nitric oxide levels in cancer. Nitric oxide 2008, 19 (2), 73-76.

70. Thomas, D. D.; Espey, M. G.; Ridnour, L. A.; Hofseth, L. J.; Mancardi, D.; Harris, C. C.; Wink, D. A., Hypoxic inducible factor 1 $\alpha$, extracellular signalregulated kinase, and p53 are regulated by distinct threshold concentrations of 
nitric oxide. Proceedings of the National Academy of Sciences of the United States of America 2004, 101 (24), 8894-8899.

71. Borutaite, V.; Brown, G. C., S-nitrosothiol inhibition of mitochondrial complex I causes a reversible increase in mitochondrial hydrogen peroxide production. Biochimica et Biophysica Acta (BBA)-Bioenergetics 2006, 1757 (5), 562-566.

72. Vaughn, M. W.; Kuo, L.; Liao, J. C., Estimation of nitric oxide production and reactionrates in tissue by use of a mathematical model. American Journal of Physiology-Heart and Circulatory Physiology 1998, 274 (6), H2163-H2176.

73. Reynolds, M. M.; Frost, M. C.; Meyerhoff, M. E., Nitric oxide-releasing hydrophobic polymers: preparation, characterization, and potential biomedical applications. Free Radic Biol Med 2004, 37 (7), 926-36.

74. $\quad$ Fleser, P. S.; Nuthakki, V. K.; Malinzak, L. E.; Callahan, R. E.; Seymour, M. L.; Reynolds, M. M.; Merz, S. I.; Meyerhoff, M. E.; Bendick, P. J.; Zelenock, G. B., Nitric oxide-releasing biopolymers inhibit thrombus formation in a sheep model of arteriovenous bridge grafts. Journal of vascular surgery 2004, 40 (4), 803811.

75. Zhang, H.; Annich, G. M.; Miskulin, J.; Stankiewicz, K.; Osterholzer, K.; Merz, S. I.; Bartlett, R. H.; Meyerhoff, M. E., Nitric oxide-releasing fumed silica particles: synthesis, characterization, and biomedical application. J Am Chem Soc 2003, 125 (17), 5015-24.

76. Hetrick, E. M.; Prichard, H. L.; Klitzman, B.; Schoenfisch, M. H., Reduced foreign body response at nitric oxide-releasing subcutaneous implants. Biomaterials 2007, 28 (31), 4571-80.

77. Shin, J. H.; Schoenfisch, M. H., Improving the biocompatibility of in vivo sensors via nitric oxide release. The Analyst 2006, 131 (5), 609.

78. Major, T. C.; Brant, D. O.; Reynolds, M. M.; Bartlett, R. H.; Meyerhoff, M. E.; Handa, H.; Annich, G. M., The attenuation of platelet and monocyte activation in a rabbit model of extracorporeal circulation by a nitric oxide releasing polymer. Biomaterials 2010, 31 (10), 2736-2745. 
79. MacMicking, J.; Xie, Q.-w.; Nathan, C., Nitric oxide and macrophage function. Annual review of immunology 1997, 15 (1), 323-350.

80. Meldrum, D. R.; Shames, B. D.; Meng, X.; Fullerton, D. A.; McIntyre, R. C.; Grover, F. L.; Harken, A. H., Nitric oxide downregulates lung macrophage inflammatory cytokine production. The Annals of thoracic surgery 1998, 66 (2), 313-317.

81. Salvemini, D.; Masini, E.; Pistelli, A.; Mannaioni, P. F.; Vane, J., Nitric oxide: a regulatory mediator of mast cell reactivity. Journal of cardiovascular pharmacology 1991, 17, S258\&hyhen.

82. Masini, E.; Salvemini, D.; Pistelli, A.; Mannaioni, P.; Vane, J., Rat mast cells synthesize a nitric oxide like-factor which modulates the release of histamine. Agents and actions 1991, 33 (1-2), 61-63.

83. Eastmond, N. C.; Banks, E.; Coleman, J. W., Nitric oxide inhibits IgE-mediated degranulation of mast cells and is the principal intermediate in IFN-gammainduced suppression of exocytosis. The Journal of Immunology 1997, 159 (3), 1444-1450.

84. Tousoulis, D.; Kampoli, A.-M.; Tentolouris Nikolaos Papageorgiou, C.; Stefanadis, C., The role of nitric oxide on endothelial function. Current vascular pharmacology 2012, 10 (1), 4-18.

85. Oppenheim, J. J., Cytokines: past, present, and future. International journal of hematology 2001, 74 (1), 3-8.

86. Cooke, M., John P; Dzau, M., Victor J, Nitric oxide synthase: role in the genesis of vascular disease. Annual review of medicine 1997, 48 (1), 489-509.

87. Fingerle, J.; Johnson, R.; Clowes, A.; Majesky, M.; Reidy, M., Role of platelets in smooth muscle cell proliferation and migration after vascular injury in rat carotid artery. Proceedings of the National Academy of Sciences 1989, 86 (21), 8412-8416.

88. Garg, U. C.; Hassid, A., Nitric oxide-generating vasodilators and 8-bromocyclic guanosine monophosphate inhibit mitogenesis and proliferation of 
cultured rat vascular smooth muscle cells. Journal of Clinical Investigation 1989, 83 (5), 1774.

89. Verbeuren, T.; Jordaens, F.; Zonnekeyn, L.; Van Hove, C.; Coene, M.; Herman, A., Effect of hypercholesterolemia on vascular reactivity in the rabbit. I. Endothelium-dependent and endothelium-independent contractions and relaxations in isolated arteries of control and hypercholesterolemic rabbits. Circulation research 1986, 58 (4), 552-564.

90. Ludmer, P. L.; Selwyn, A. P.; Shook, T. L.; Wayne, R. R.; Mudge, G. H.; Alexander, R. W.; Ganz, P., Paradoxical vasoconstriction induced by acetylcholine in atherosclerotic coronary arteries. New England Journal of Medicine 1986, 315 (17), 1046-1051.

91. Chin, J. H.; Azhar, S.; Hoffman, B. B., Inactivation of endothelial derived relaxing factor by oxidized lipoproteins. Journal of Clinical Investigation 1992, $89(1), 10$.

92. Minor Jr, R. L.; Myers, P. R.; Guerra Jr, R.; Bates, J. N.; Harrison, D., Dietinduced atherosclerosis increases the release of nitrogen oxides from rabbit aorta. Journal of Clinical Investigation 1990, 86 (6), 2109.

93. Campeau, L.; Enjalbert, M.; Lesperance, J.; Vaislic, C.; Grondin, C.; Bourassa, M., Atherosclerosis and late closure of aortocoronary saphenous vein grafts: sequential angiographic studies at 2 weeks, 1 year, 5 to 7 years, and 10 to 12 years after surgery. Circulation 1983, 68 (3 Pt 2), II1-7.

94. West, N. E.; Qian, H.; Guzik, T. J.; Black, E.; Cai, S.; George, S. E.; Channon, K. M., Nitric oxide synthase (nNOS) gene transfer modifies venous bypass graft remodeling effects on vascular smooth muscle cell differentiation and Superoxide production. Circulation 2001, 104 (13), 1526-1532.

95. Wink, D.; Vodovotz, Y.; Cook, J.; Krishna, M.; Kim, S.; Coffn, D.; DeGraff, W.; Deluca, A.; Liebmann, J.; Mitchell, J., Reviews-The Role of Nitric Oxide Chemistry in Cancer Treatment. Biochemistry-New York-English Translation of Biokhimiya 1998, 63 (7), 802-809. 
96. Koskela, L. R.; Poljakovic, M.; Ehrén, I.; Wiklund, N. P.; de Verdier, P. J., Localization and expression of inducible nitric oxide synthase in patients after BCG treatment for bladder cancer. Nitric Oxide 2012, 27 (3), 185-191.

97. Gallo, O.; Fini-Storchi, I.; Vergari, W. A.; Masini, E.; Morbidelli, L.; Ziche, M.; Franchi, A., Role of nitric oxide in angiogenesis and tumor progression in head and neck cancer. Journal of the National Cancer Institute 1998, 90 (8), 587-596.

98. Morbidelli, L.; Chang, C.-H.; Douglas, J. G.; Granger, H. J.; Ledda, F.; Ziche, M., Nitric oxide mediates mitogenic effect of VEGF on coronary venular endothelium. American Journal of Physiology-Heart and Circulatory Physiology 1996, 270 (1), H411-H415.

99. Contreras, D. L.; Robles, H. V.; Romo, E.; Rios, A.; Escalante, B., The role of nitric oxide in the post-ischemic revascularization process. Pharmacology \& therapeutics 2006, 112 (2), 553-563.

100. de Mel, A.; Murad, F.; Seifalian, A. M., Nitric oxide: a guardian for vascular grafts? Chemical reviews 2011, 111 (9), 5742-5767.

101. Griess, P., Bemerkungen zu der Abhandlung der HH. Weselsky und Benedikt „Ueber einige Azoverbindungen”. Berichte der deutschen chemischen Gesellschaft 1879, 12 (1), 426-428.

102. Tracey, W. R., Spectrophotometric detection of nitrogen oxides using azo dyes. Neuroprotocols 1992, 1 (2), 125-131.

103. Bedioui, F.; Villeneuve, N., Electrochemical nitric oxide sensors for biological samples-principle, selected examples and applications. Electroanalysis 2003, 15 (1), 5-18.

104. Coneski, P. N.; Schoenfisch, M. H., Nitric oxide release: part III. Measurement and reporting. Chem. Soc. Rev. 2012, 41 (10), 3753-3758.

105. Kitamura, Y.; Uzawa, T.; Oka, K.; Komai, Y.; Ogawa, H.; Takizawa, N.; Kobayashi, H.; Tanishita, K., Microcoaxial electrode for in vivo nitric oxide measurement. Analytical chemistry 2000, 72 (13), 2957-2962. 
106. McMurtry, M.; Kim, D.; Dinh-Xuan, T.; Archer, S., Measurement of nitric oxide, nitrite and nitrate using a chemiluminescence assay: an update for the year 2000. Analusis 2000, 28 (6), 455-465.

107. Li, C. M.; Zang, J.; Zhan, D.; Chen, W.; Sun, C. Q.; Teo, A. L.; Chua, Y.; Lee, V.; Moochhala, S., Electrochemical detection of nitric oxide on a SWCNT/RTIL composite gel microelectrode. Electroanalysis 2006, 18 (7), 713-718.

108. Tarpey, M. M.; Fridovich, I., Methods of Detection of Vascular Reactive Species: Nitric Oxide, Superoxide, Hydrogen Peroxide, and Peroxynitrite. Circulation Research 2001, 89 (3), 224-236. 


\section{Chapter 2 : Controlled Nitric Oxide Release from Covalently Linked S-Nitroso- $N$-Acetyl-D-Penicillamine to Polyvinyl Chloride (SNAP-PVC)}

\subsection{Introduction}

PVC is the most widely used polymeric material used in medical applications ranging from blood bags, heart lung bypass sets, endotracheal tubes, and many other medical related materials ${ }^{1}$. Around $25 \%$ of all biomedical devices contain some form of PVC due to its wide range of mechanical property variability with plasticizer additives ${ }^{2}$. Some of the plasticizers that can be used in PVC are toxic such as di-(2-ethylhexyl phthalate) (DEHP), and are able to leech out of the polymer into the blood ${ }^{3,4}$. Although these ester based plasticizers are toxic, much safer PVC plasticizers have been developed to be used for blood contacting purposes. Examples of non-toxic plasticizers that are blendable with PVC are citrates and sebacates ${ }^{1,5}$. Plasticizer leeching can also be controlled by performing surface modifications to the polymer. A common technique used is surface crosslinking, which acts as a barrier to prevent the escape of any unwanted chemicals ${ }^{6}$. This can also be useful for an NO releasing blendable compound that is designed to only release NO at a certain area and time and not diffuse out of the material. PVC is also has a very low cost to manufacture compared to other medical grade polymers and has a strong resistance to external chemical components. Being able to chemically modify a polymer is also an important characteristic of a polymer. The free chlorine reactive groups allow for the attachment of other chemical components to further enhance the surface chemistry of PVC. Chemically modifying the plasticizers used within the polymer is also an option for attaching small molecules which is a popular strategy for NO releasing polydimethylsiloxane (PDMS), a well characterized NO donating material ${ }^{7,8} .{ }^{1}$

\footnotetext{
${ }^{1}$ Material in this chapter is in the process of being submitted for publication.
} 
Polymers can be synthesized to mimic the endothelium's NO release levels to prevent unwanted foreign body response. A novel NO releasing PVC based polymer is described and shown to have tunable NO releasing properties depending on the trigger mechanism. NO releasing PVC has been reported by blending N-diazeniumdiolates within the polymer matrix and covalently binding it to a modified version of the polymer $^{9,10}$. N-diazeniumdiolates do not have the controllability that RSNOs contain as they are limited to only passively release their NO reservoir over a short time ${ }^{11}$. Using a SNAP based system, a material is able to have a tunable control of NO release for a variety of situations along with the possibility to create a passive release system.

When developing an NO releasing polymer, the capability of NO diffusion through a polymer matrix is important. NO permeability through PVC has been characterized using Fick's law of diffusion where the equation is as follows:

$$
\ln \left(\frac{C_{o}}{C_{o}-C}\right)=\frac{D A}{l V} t
$$

Where $\mathrm{C}$ is the concentration of $\mathrm{NO}, \mathrm{D}$ is the diffusion coefficient, $\mathrm{A}$ is the area being diffused through, 1 is the path length, $\mathrm{V}$ is the chamber volume, and $\mathrm{t}$ is the time. Although its diffusion kinetics are not as good as silicone rubber, it is greatly enhanced through the addition of the plasticizer dioctyl sebacate ${ }^{12}$. This needs to be taken into consideration when a system with mass transfer of NO through PVC or any other polymer is designed. NO releasing materials that are blended within a polymer matrix while in contact with blood will need to be designed with a layer that prevents diffusion of the NO donor out of the material, but still allows the permeability of NO itself. This diffusion rate can be tailored to the dosage of passive NO being administered as well. Having an additional parameter available to modulate the rate of NO release could be beneficial.

Herein, a synthetic route is described where (PVC) is aminated to a specified extent and then further modified by covalently linking S-nitroso- $N$-acetyl-D-penicillamine (SNAP) groups to the free primary amine sites to form a nitric oxide releasing polymer (SNAP- 
PVC). Controllable release of NO from SNAP-PVC is described using photoinitiation from light emitting diodes (LEDs). Ion mediated NO release is also demonstrated as another pathway to give a passive mechanism for NO delivery. The large range of NO fluxes obtainable from the SNAP-PVC films demonstrates the potential uses in mediating unwanted inflammatory response in blood and tissue contacting devices.

\subsection{Experimental Details}

\subsubsection{Materials}

Polyvinyl chloride (average $M_{w}=233,000, M_{n}=99,000$ ), ethylenediamine, 5, 5'dithiobis (2-nitrobenzoic acid), triethylamine, 1,4,8,11-tetraazacyclotetradecane, $\mathrm{N}$ acetyl- $D$-penicillamine (Fluka), and concentrated hydrochloric acid were obtained from Sigma-Aldrich (St. Louis, MO, USA). ATTO-TAG ${ }^{\mathrm{TM}}$ FQ reagent was purchased from Invitrogen (Grand Island, NY, USA). Tert-butyl nitrite ( $90 \%$ technical grade, Acros Organics) was purchased from Fisher Scientific. Magnesium sulfate, copper (II) bromide, L-ascorbic acid sodium salt, and acetic anhydride (Alfa Aesar) were purchased from VWR (West Chester, PA, USA).

\subsubsection{Synthesis of NAP-thiolactone}

The procedure to synthesize a self-protected N-acetyl-D-penicillamine (NAP) thiolactone was accomplished according to the procedure from Moynihan and Robert ${ }^{13}$ (Figure 2.1). $5 \mathrm{~g}$ of N-acetyl-D-penicillamine was dissolved in $10 \mathrm{~mL}$ of pyridine while $10 \mathrm{~mL}$ of acetic anhydride and $10 \mathrm{~mL}$ of pyridine were mixed in a separate container. The solutions were both cooled in an ice bath for $1 \mathrm{~h}$ before being combined. The mixture was allowed to stir at room temperature for approximately $24 \mathrm{~h}$ until the solution turned light red. The solution was rotary evaporated at $45^{\circ} \mathrm{C}$ until the solution stopped boiling. The temperature was then increased to $60^{\circ} \mathrm{C}$ until most of the solvent was evaporated to obtain an orange viscous liquid. The resulting product was then dissolved in $20 \mathrm{~mL}$ of chloroform and washed and extracted three times with $20 \mathrm{~mL}$ of 
$1 \mathrm{M} \mathrm{HCl} . \mathrm{MgSO}_{4}$ was added to the chloroform solution to remove any water and was then removed by vacuum filtration. The chloroform was removed by rotary evaporation at room temperature and the resultant crystals were collected and rinsed with hexanes and filtered. Light yellow/white colored crystals were recovered and vacuum dried overnight.

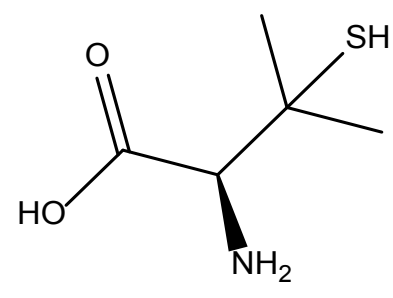

$\mathrm{N}$-acetyl-DL-penicillamine

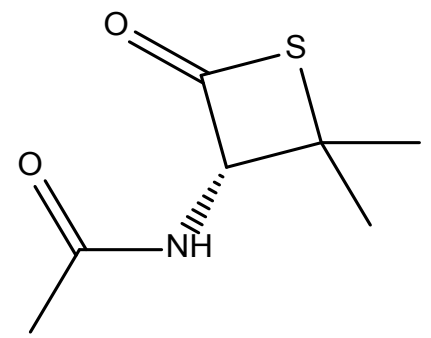

$\mathrm{N}$-acetylpenicillamine thiolactone

Figure 2.1. Synthesis of thiolactone self-protected N-acetyl-D-penicillamine (NAPthiolactone). The base molecule N-acetyl-D-penicillamine is first dissolved into chilled pyridine and acetic anhydride where it is then allowed to stir for approximately 24 hours. The pyridine is then rotary evaporated off and the resulting viscous liquid is dissolved in chloroform and extracted three times with $1 \mathrm{M} \mathrm{HCl}$. Any excess water is removed by placing anhydrous magnesium sulfate into the organic solution and filtered off. The chloroform is then rotary evaporated off at room temperature and rinsed with hexanes to give a white/light yellow crystalline product.

\subsubsection{Synthesis of SNAP-PVC}

The overall schematic to synthesize SNAP-PVC is shown below in Figure 2.2. Aminated PVC was first synthesized using a modified procedure from Tinkilic et al ${ }^{14}$. $2.5 \mathrm{~g}$ of PVC was suspended in $50 \mathrm{~mL}$ of methanol, $11 \mathrm{~mL}$ of triethylamine, and heated to $60^{\circ} \mathrm{C} .15 .25 \mathrm{~mL}$ of ethylenediamine was added once the solution reached temperature. The reaction mixture was allowed to reflux for at varied time points $(1,2$, and 4 hours) at $60^{\circ} \mathrm{C}$. The resulting light yellow polymer powder was then filtered and washed thoroughly with water, methanol, $1 \mathrm{M} \mathrm{HCl}$, methanol, and water in that order before being dried under vacuum. 
$200 \mathrm{mg}$ of the synthesized PVC-NH $\mathrm{N}_{2}$ was dissolved in $10 \mathrm{~mL}$ of anhydrous $\mathrm{N}, \mathrm{N}-$ dimethylacetamide (DMAC). $60 \mathrm{mg}$ of NAP-thiolactone was then added to the mixture and allowed to stir overnight. $2 \mathrm{~mL}$ of the NAP-PVC solution was taken out and nitrosated using $0.5 \mathrm{~mL}$ of t-butyl nitrite. The t-butyl nitrite was first chelated of any copper stabilizer using a $30 \mathrm{mM}$ aqueous cyclam solution $(1,4,8,11$ tetraazacyclotetradecane). For the chelation step, approximately $3 \mathrm{~mL}$ of t-butyl nitrite and $5 \mathrm{~mL}$ of $30 \mathrm{mM}$ cyclam were mixed and stirred vigorously. The cyclam was then removed and the t-butyl nitrite was rewashed with more cyclam a total of three times before the cleaned t-butyl nitrite was extracted and stored at $2^{\circ} \mathrm{C}$. The light yellow/clear solution of PVC-thiolactone turns a light green-yellow color after allowing it to react with t-butyl nitrite for 24 hours. The SNAP-PVC solution was then cast into polytetrafluoroethylene (PTFE) rings and left to air dry overnight to form a thin layered polymer film. The films can also be dried under a vacuum as moisture will cause the films to be less transparent, hindering the ability the LED can trigger photoinitiated NO release. 


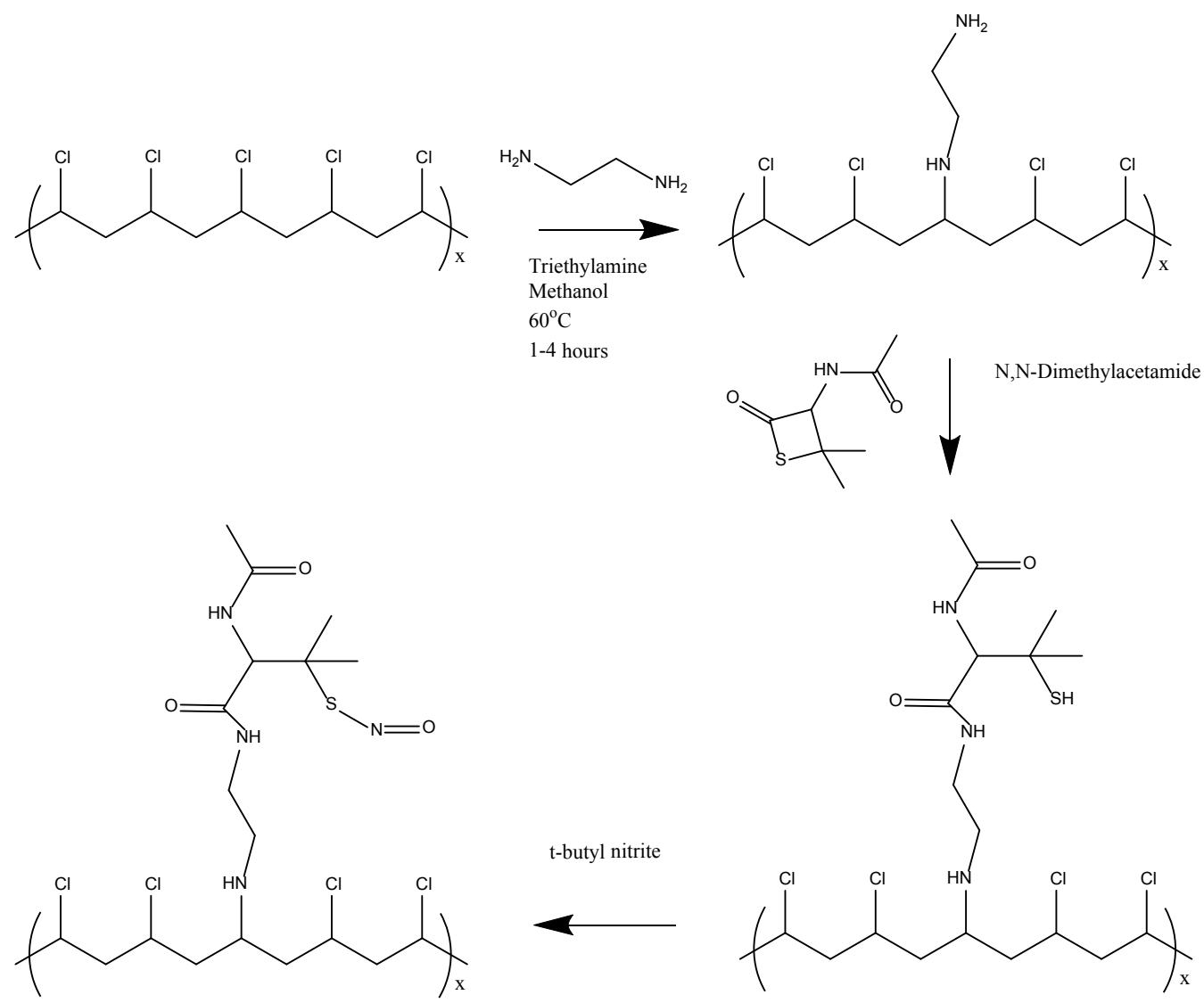

Figure 2.2. Synthesis route of SNAP-PVC. PVC is first modified to contain reactive primary amine site by suspending PVC resin in methanol, triethylamine, and ethylenediamine while refluxing at $60^{\circ} \mathrm{C}$. This attaches the ethylenediamine to the PVC backbone. The free primary amine can then react with NAP-thiolactone to form a NAPPVC polymer. Nitrosation of the free thiol group was then done through the reaction of t-butyl nitrite to form SNAP-PVC.

\subsubsection{Polymer characterization}

The quantification of the degree of amination of the PVC was done by using the ATTOTAG FQ test for primary amines. Fluorescence testing was done using a 96-well plate reader (BioTek Instruments) and the amount of primary amines was determined using a glycine calibration curve. The fluorescent tag is maximally excited at $450 \mathrm{~nm}$ with an emission maximum at $550 \mathrm{~nm}^{15}$. Once the aminated PVC reacts with NAP-thiolactone, 
a free thiol is exposed on the polymer backbone. Ellman's test for thiols was done to quantify the amount of thiols grafted onto the polymer using 5, 5'-dithiobis (2nitrobenzoic acid) (DNTB) following a modified protocol developed by George Ellman $^{18}$. Tris-buffer was substituted with PBS $(\mathrm{pH}=7.4)$. Absorbance was then taken at $412 \mathrm{~nm}$ after the reaction with 5,5'-dithio-bis-2-nitrobenzoic acid (DTNB) using a 96well plate reader. As the reaction progressed, FTIR was done to characterize the important polymer functional groups (Mattson Genesis II).

\subsubsection{Quantification of primary amines}

Primary amines are able to be accurately quantified through their reaction with 3-(2furoyl)quinoline-2-carboxaldehyde (FQ) to form a fluorescent isoindole product ${ }^{17}$ (Figure 2.3). This is an extremely sensitive method, able to detect amines in the attomole $\left(10^{-18}\right)$ range. The development of this detection method was originally made to quantify trace amounts of peptides and amino acids that could not be identified through traditional methods like liquid chromatography and capillary electrophoresis. The application can be applied to synthetic materials with a primary amine present. The reaction that occurs with $\mathrm{FQ}$, potassium cyanide $(\mathrm{KCN})$, and the primary amine containing sample is shown in the schematic below. 


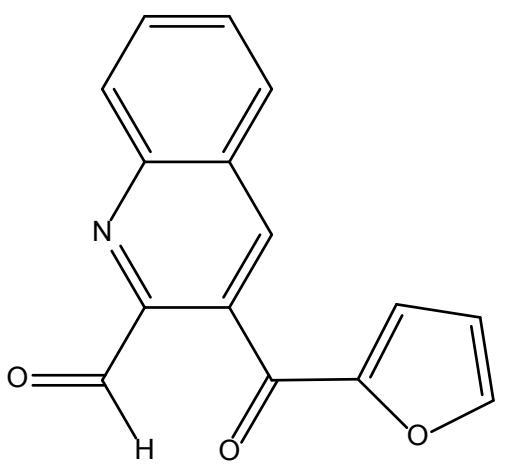

$+\quad \mathrm{R}-\mathrm{NH}_{2}+\mathrm{KCN}$

FQ

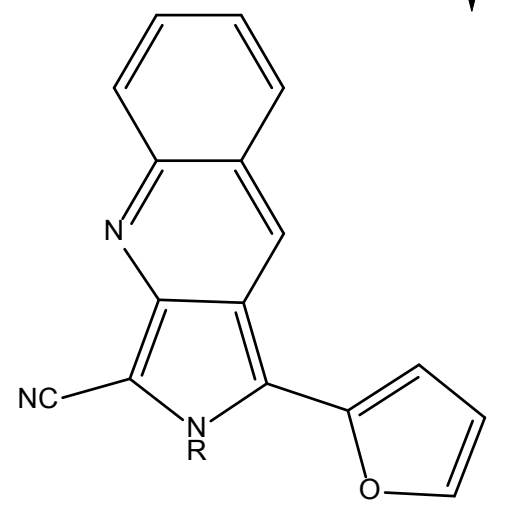

Fluorescent Indole Product

Figure 2.3. Mechanism for the fluorescent detection of primary amines using the ATTO-TAG FQ amine derivation kit.3-(2-furoyl)quinoline-2-carboxaldehyde (FQ) in the presence of a potassium cyanide will react with a primary amine site to form a fluorescent product. This product has an excitation wavelength of $450 \mathrm{~nm}$ and an emission maximum of $550 \mathrm{~nm}$, which can be quantified to give an accurate measurement of primary amines in a material when compared to a calibration curve.

For a control, glycine- $\mathrm{HCl}$ was used and diluted to multiple concentrations to generate a calibration curve. Each well to be tested on the 64-well fluorescent plate contained $10 \mu \mathrm{L}$ of $10 \mathrm{mM}$ CBQCA, $20 \mu \mathrm{L}$ of $10 \mathrm{mM} \mathrm{KCN}$, and $10 \mu \mathrm{L}$ of sample. The reaction was allowed to sit on a shaker plate for 1 hour while protected from light before being tested in the plate reader with an excitation wavelength of $450 \mathrm{~nm}$ with an emission maximum at $550 \mathrm{~nm}$. 


\subsubsection{Quantification of thiols}

Thiol quantification through the use of DTNB directly cleaves the DTNB molecule into two TNB anions, where one of the free molecules reacts with the thiol being measured (Figure 2.4). The other TNB anion is what is measured by the spectrophotometer at 412 nm. Cysteine was used to generate a calibration curve up to an absorbance of 1.0. Any absorbance above 1.0 would not be measured as Beer's Law cannot accurately predict concentrations above those values.<smiles>[R]S[SH+]</smiles><smiles>[R]SSc1ccc([N+](=O)[O-])c(C(=O)O)c1</smiles><smiles>O=C(O)c1cc(S)ccc1[N+](=O)[O-]</smiles>

Measured TNB Product

Figure 2.4. Mechanism for the detection of free thiols using Ellman's assay. 5,5'-dithiobis-2-nitrobenzoic acid (DTNB) has its disulfide bond cleaved in the presence of free thiols. The free thiol then forms another disulfide bond with one part of the DTNB while the measured product is emitted into solution and is quantifiable at $412 \mathrm{~nm}$ when compared to a calibration standard.

\subsubsection{Nitric oxide release by photoinitiation}


The light source used for photoinitiation of the SNAP-PVC films were $460 \mathrm{~nm}$ blue VAOL-5GSBY4 LEDs obtained from Mouser Electronics Inc (Mansfield, TX, USA). A $130 \Omega$ resistor was hooked up in series with the LED and a variable voltage source was applied to the system. The emission spectra and relative intensity of the LED with respect to drive current is shown to be linear over the currents being used to power the LED for the study and was characterized by Starrett et $\mathrm{al}^{16}$.

\subsubsection{Nitric oxide measurements}

Nitric oxide release from the polymer was directly measured by the chemiluminescent reaction of NO with ozone using a Sievers 280i Nitric Oxide Analyzer (GE Instruments). SNAP-PVC films with a diameter of $5.5 \mathrm{~mm}$ were placed inside an amber

glass sample holder with an inlet nitrogen sweep gas flowing at $200 \mathrm{~mL} \mathrm{~min}^{-1}$. The LED was mounted in the top of the sample holder $5 \mathrm{~cm}$ above the film to be tested. Varying voltage levels were used $(0,3,4.5,6,7.5$ volts $)$ at $130 \Omega$ to demonstrate the control of the nitric oxide flux from the polymer film. Total NO release of the polymer films was done by cleaving the sulfur-nitroso bond through copper mediated decomposition following a procedure developed by Frost et $\mathrm{al}^{17}$. A solution of $10 \mathrm{mM} \mathrm{CuBr}_{2}$ and a $100 \mathrm{mM}$ solution of L-ascorbic acid sodium salt were used for the NO quantification. 2 $\mathrm{mL}$ of the $\mathrm{CuBr}_{2}$ solution are placed in a glass sample holder containing a weighed SNAP-PVC film. After 5 minutes, $500 \mu \mathrm{L}$ of the $100 \mathrm{mM}$ L-ascorbic acid solution is then added to reduce the $\mathrm{Cu}^{2+}$ ions to $\mathrm{Cu}^{+}$, enhancing the sulfur-nitroso bond cleaving. The test was continued until all of the NO reservoir was depleted.

\subsection{Results and Discussion}

\subsubsection{FTIR analysis}

Each step from the reaction schematic shown in the SNAP-PVC synthesis route is verified using FTIR which is seen in Figure 2.5. After attaching ethylenediamine to the 45 
PVC backbone, primary amines and secondary amines are present in the spectrum which is seen in the N-H bending at $1641 \mathrm{~cm}^{-1}$. The attachment of NAP-thiolactone to the free primary amine sites shows the formation of NAP-PVC as the presence of a carbonyl peak is present at $1750 \mathrm{~cm}^{-1}$.

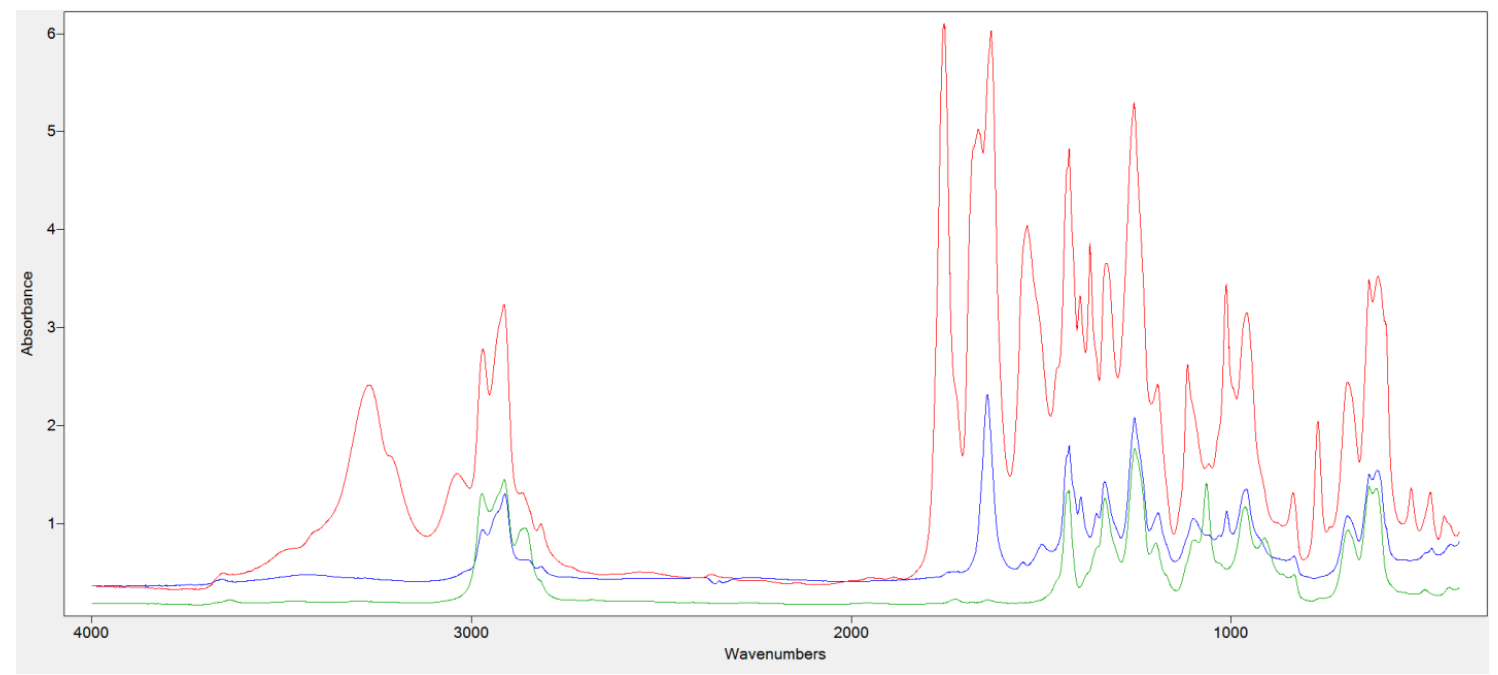

Figure 2.5. FTIR spectrum of PVC (green), $\mathrm{PVC}-\mathrm{NH}_{2}$ (blue), and NAP-PVC (red). Once ethylenediamine is attached to $\mathrm{PVC}$, the presence of N-H bending is seen at 1641 $\mathrm{cm}^{-1}$. The attachment of NAP-thiolactone to the free amine shows the appearance of the amide carbonyl peak at $1750 \mathrm{~cm}^{-1}$ along with the secondary amine stretching around the $3300 \mathrm{~cm}^{-1}$ region.

\subsubsection{Quantification Results}

Quantification of the amination of PVC was verified through using the ATTO-TAG FQ test for amines. Once the NAP-thiolactone ring opens and reacts with the primary amine sites on the polymer backbone, free thiol groups are exposed. To identify the extent to 
which this reaction is occurring, Ellman's test for thiols was done. The quantification of primary amine and thiol functional groups were normalized per milligram of polymer. Primary amine quantification showed $0.201 \pm 0.013 \mu \mathrm{mol} / \mathrm{mg}$ of PVC-NH${ }_{2}$. Thiol quantification showed $0.153 \pm 0.009 \mu \mathrm{mol} / \mathrm{mg}$ of NAP-PVC. To verify the capacity, copper mediated NO release was performed on the nitrosated polymer (SNAP-PVC) and showed the NO released to be $0.0392 \pm 0.004 \mu \mathrm{mol}$ per milligram of polymer, which is a $25.6 \% \mathrm{NO}$ addition efficiency of the NAP-PVC compound.

\subsubsection{Nitric oxide release results}

The physiological NO flux for healthy vasculature is estimated to be in the range of 0.5 $-4 \times 10^{-10} \mathrm{~mol} \mathrm{~cm}^{-2} \mathrm{~min}^{-1}$, which is achieved from the SNAP-PVC films at certain intensities of light. The NO flux varies depending on the location in the body. Vasculature with higher sheer stress will promote more NO release from vascular endothelial cells ${ }^{19}$. After damaging the vascular walls from an implanted device, more NO release may be initially required to prevent unwanted immune response and neointimal hyperplasia in the affected $\operatorname{area}^{20}$. Developing a material that is able to adjust the NO flux in real-time increases the number of applications a hydrophobic polymer coating could have in a medical setting.

The NO release profile from SNAP-PVC films (diameter $5.5 \mathrm{~mm}$, thickness $0.1 \mathrm{~mm}$ ) at varying drive current applied to a $470 \mathrm{~nm}$ wavelength LED is shown in Figure 2.6. Nitrogen sweep gas carried the NO released by photoinitiation to the NOA to give a stair step profile based on the voltage supplied to the LED. The profile shows the wide range of control that the polymer is able to obtain. As the LED is adjusted, there is little decay in the NO being released at each level showing the stability of the NO reservoir within the polymer. 


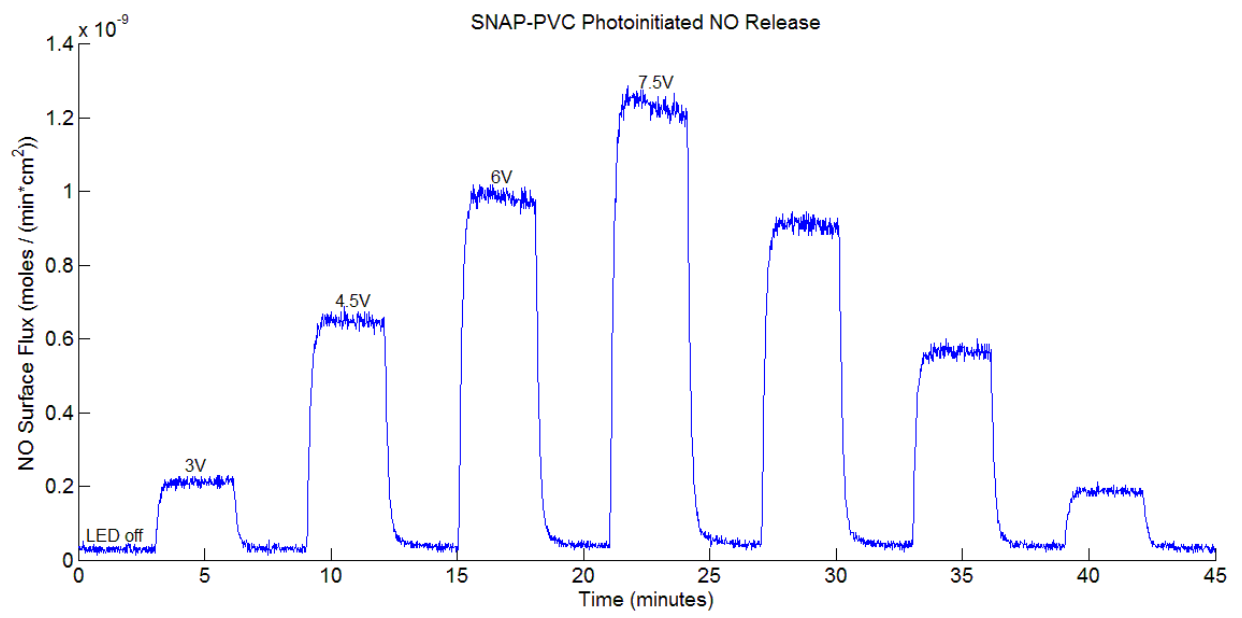

Figure 2.6. Controlled NO release profile of SNAP-PVC at varying LED voltages. A baseline is first obtained with the LED off, and is then turned on with a $3 \mathrm{~V}$ supply at $130 \Omega$. It is then turned off again until the baseline is reached again before being supplied with an additional $1.5 \mathrm{~V}$ while holding the resistance constant. Once $7.5 \mathrm{~V}$ is reached, the processed is repeated but with a decreasing supply of $1.5 \mathrm{~V}$.

NO release of SNAP-PVC in phosphate buffered saline (PBS) at $37^{\circ} \mathrm{C}$ was also tested to show the release under physiological conditions. Ions are able to penetrate the polymer film and interact with the reactive SNAP groups as PVC is a popular polymer used in ion selective electrodes ${ }^{21}$. This provides another pathway to stimulate the NO response for in vivo applications as other methods like photoinitiation could cause complications when implemented. In some scenarios, having a level of passive release over a short period of time may be the desired route of NO delivery. This follows a similar release profile as seen with $\mathrm{N}$-diazeniumdiolate $\mathrm{NO}$ donors, but is able to release lower amounts over a longer period of time. The passive release of SNAP-PVC in PBS at $37^{\circ} \mathrm{C}$ is shown in Figure 2.7. 


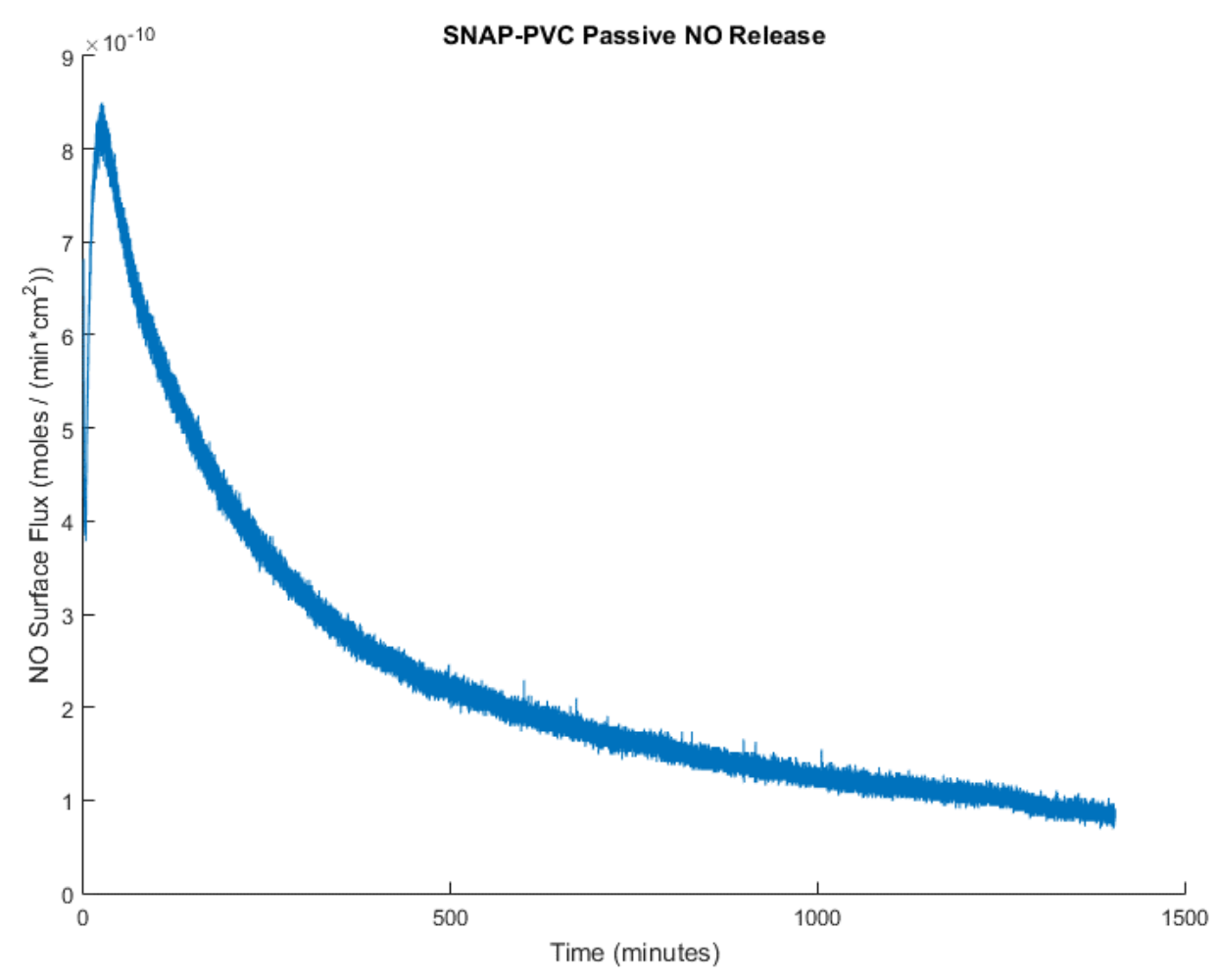

Figure 2.7. NO release profile of SNAP-PVC placed in PBS at $37^{\circ} \mathrm{C} .5 .5 \mathrm{~mm}$ diameter, $100 \mu \mathrm{m}$ thick films were submerged in $2 \mathrm{~mL}$ of PBS while stirring at physiological temperature to demonstrate SNAP-PVC's passive NO release. This profile is similar to NONOates but is able to persist a lower and more constant release.

The NO capacity and release demonstrated by SNAP-PVC could potentially be much larger as there are many unreacted chlorine sites on the backbone of the polymer. A longer reaction time with ethylenediamine could be implemented to achieve a higher degree of amination, but complications with crosslinking and solubility occur as PVC becomes more dechlorinated ${ }^{22}$. This limits the amount of NO storage capacity of PVC through using a component like ethylenediamine as an aminating agent. PVC also becomes darker in color the more dechlorinated it becomes, which would hinder the NO release by photoinitiation as light penetration through the film will be limited. NO release from films reacting with ethylenediamine from 1 to 4 hours was tested, and 2 hours was found to have the highest capacity and release ability of NO, which is 
demonstrated in Figure 2.8.

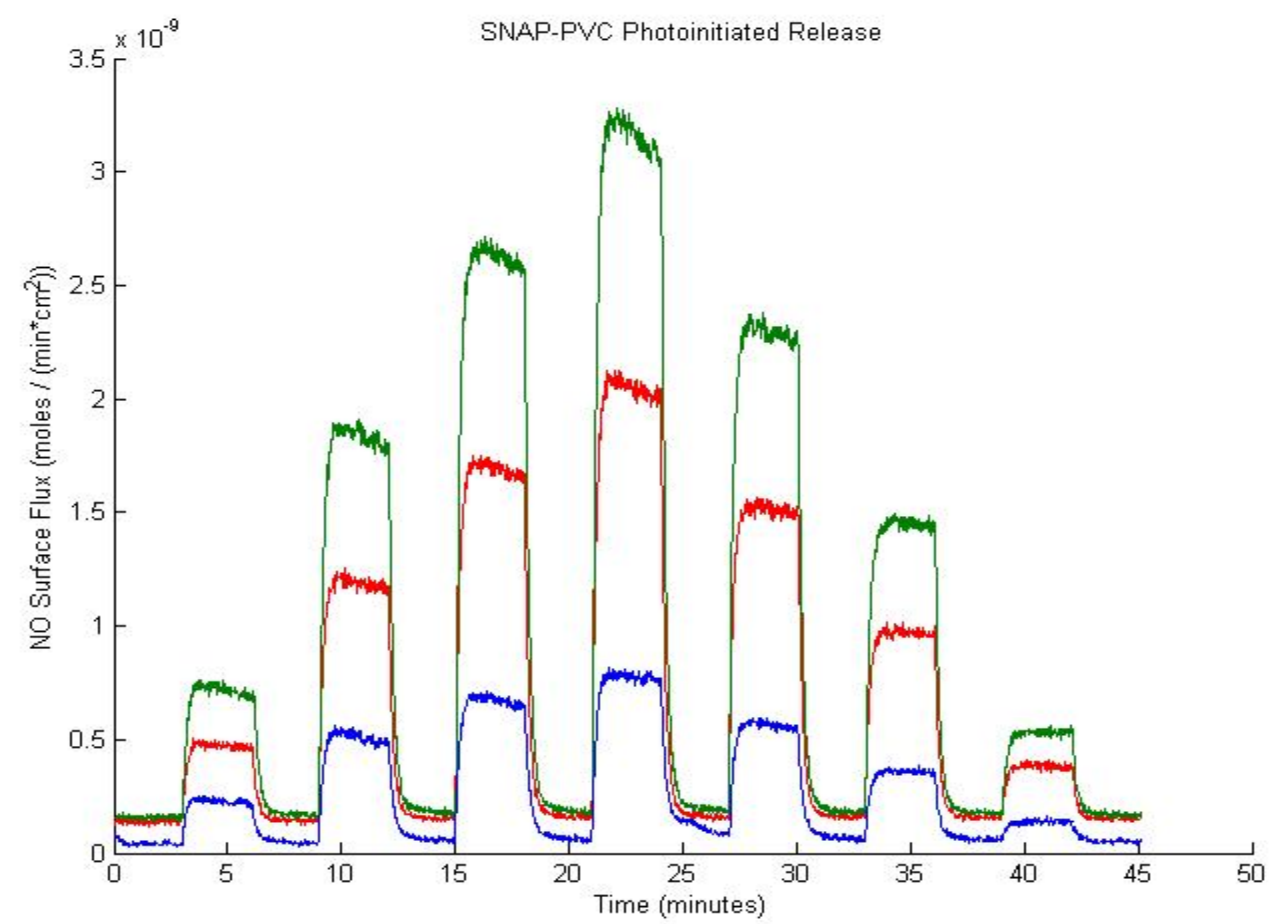

Figure 2.8. SNAP-PVC photoinitiated NO release at $37^{\circ} \mathrm{C}$ in nitrogen at different synthesis reaction times. The reaction times with ethylenediamine (EDA) were 1 hour (red), 2 hours (green), and 4 hours (blue). The same LED and stepwise voltage increases seen in Figure 2.6 were used here. As the reaction time of EDA with PVC increases, more crosslinking between the polymer chains occurs. This crosslinking limits the amount of free amine sites to be reacted with NAP-thiolactone while also causing the PVC to become a darker yellow color, limiting the release from the LED.

Total NO release was also determined for the polymer films with varied reaction times to ensure that the 2 hour EDA reacted SNAP-PVC was the most optimized reaction time to achieve the highest NO loading in the material. To do this, the three films were all placed into a reaction chamber where a high intensity UV light was shined onto the films until no visible NO release was detected. Figure 2.9 shows that the higher 4 hour reaction time SNAP-PVC was releasing the lowest amount of NO while the 2 hour reaction time SNAP-PVC released the highest. This displays the limit of NO release that can be done using this method, as the crosslinking interferes too much passed a certain amination point. This is further proven in Table 2.1, where the increase in 
ethylenediamine reaction time does show to increase the nitrogen content, but a decrease in primary amines at the 4 hour reaction time. Further improvements could be made to increase the capacity by using a self-protected aminating agent instead of a difunctional aminating agent. This would allow the attachment of the reactive molecule to the backbone of the PVC chains with no crosslinking. The polymer could then be deprotected to expose the reactive group that could then be modified with SNAP.

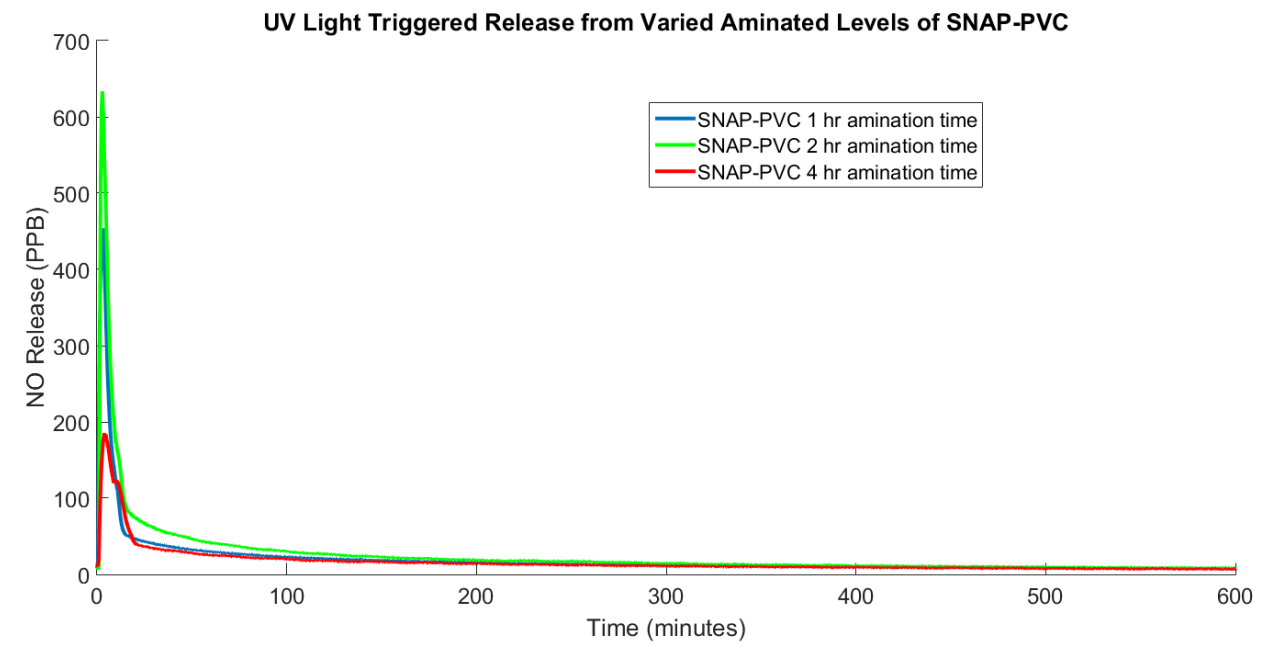

Figure 2.9. SNAP-PVC compounds with varied reaction times in EDA with triggered UV light photoinitiated for total NO release. The SNAP-PVC films were categorized into 1 hour (blue), 2 hour (green), and 4 hour (red) reaction times with EDA. Films were rinsed with DI water beforehand and vacuum dried to get rid of any excess nitrites that could interfere with the testing. SNAP-PVC with the 2 hour reaction time proved to have the highest NO storage capabilities while the higher 4 hour reaction time showed the lowest. This further proves the crosslinking kinetics that occur when reacting a polymer with a difunctional molecule over long periods of time. 
Table 2.1. FQ and CHN analysis on aminated PVC to observe the primary amine and nitrogen content as reaction time with ethylenediamine increases. As the reaction time with ethylenediamine increases, the amount of primary amines present decrease at the 4 hour reaction time point. From the CHN combustion data, there is an increase in nitrogen content, proving the crosslinking of ethylenediamine to other polymer chains the longer the solution is refluxed.

\begin{tabular}{|c|c|c|}
\hline Sample & $\begin{array}{c}\text { Primary amine } \\
\text { content }(\boldsymbol{\mu m o l} / \mathbf{m g})\end{array}$ & \%N (by mass) \\
\hline PVC 1 hour reaction time & $0.0362 \pm 0.016$ & 0.15 \\
\hline PVC 2 hour reaction time & $0.201 \pm 0.013$ & 0.167 \\
\hline PVC 4 hour reaction time & $0.0188 \pm 0.0023$ & 0.20 \\
\hline
\end{tabular}

\subsection{Conclusion}

The synthesis of NO releasing SNAP PVC was successful and the flux of NO obtained from the polymer was shown to release at a variety of physiological ranges. This could greatly improve the potential applications for blood and tissue contacting PVC based materials that are already used in medical settings. Depending on the degree of amination and light intensity administered from specific LED wavelengths, SNAP-PVC is able to be utilized to deliver specific controlled NO loading and release. The material could also be applied used as implantable device coatings to improve biocompatibility or to prevent neointimal hyperplasia of vessels. It could also be used as a base material for catheters and other PVC related medical devices, extending the duration it is allowed to stay in contact with tissue and blood before complications occur.

\subsection{References}


1. Lakshmi, S.; Jayakrishnan, A., Migration resistant, blood-compatible plasticized polyvinyl chloride for medical and related applications. Artificial organs 1998, 22 (3), 222-229.

2. Blass, C.; Jones, C.; Courtney, J., Biomaterials for blood tubing: the application of plasticised poly (vinyl chloride). The International journal of artificial organs 1992, 15 (4), 200-203.

3. Ahmed, R.; Price, S.; Grasso, P.; Hinton, R., Hepatic nuclear and cytoplasmic effects following intermittent feeding of rats with di (2-ethylhexyl) phthalate. Food and Chemical Toxicology 1990, 28 (6), 427-434.

4. Tsutsui, T.; Watanabe, E.; Barrett, J. C., Ability of peroxisome proliferators to induce cell transformation, chromosome aberrations and peroxisome proliferation in cultured Syrian hamster embryo cells. Carcinogenesis 1993, 14 (4), 611-618.

5. Krauskopf, L., Plasticizers: Types, properties, and performance. Marcel Dekker, Encyclopedia of PVC. 1988, 2, 143-261.

6. Audic, J. L.; Poncin-Epaillard, F.; Reyx, D.; Brosse, J. C., Cold plasma surface modification of conventionally and nonconventionally plasticized poly (vinyl chloride)-based flexible films: Global and specific migration of additives into isooctane. Journal of applied polymer science 2001, 79 (8), 1384-1393.

7. Zhang, H.; Annich, G. M.; Miskulin, J.; Osterholzer, K.; Merz, S. I.; Bartlett, R. H.; Meyerhoff, M. E., Nitric oxide releasing silicone rubbers with improved blood compatibility: preparation, characterization, and in vivo evaluation. Biomaterials 2002, 23 (6), 1485-1494.

8. $\quad$ Frost, M. C.; Rudich, S. M.; Zhang, H.; Maraschio, M. A.; Meyerhoff, M. E., In vivo biocompatibility and analytical performance of intravascular amperometric oxygen sensors prepared with improved nitric oxide-releasing silicone rubber coating. Analytical chemistry 2002, 74 (23), 5942-5947.

9. Major, T. C.; Brant, D. O.; Reynolds, M. M.; Bartlett, R. H.; Meyerhoff, M. E.; Handa, H.; Annich, G. M., The attenuation of platelet and monocyte activation 
in a rabbit model of extracorporeal circulation by a nitric oxide releasing polymer. Biomaterials 2010, 31 (10), 2736-2745.

10. Mowery, K. A.; Schoenfisch, M. H.; Saavedra, J. E.; Keefer, L. K.; Meyerhoff, M. E., Preparation and characterization of hydrophobic polymeric films that are thromboresistant via nitric oxide release. Biomaterials 2000, 21 (1), 9-21.

11. Davies, K. M.; Wink, D. A.; Saavedra, J. E.; Keefer, L. K., Chemistry of the diazeniumdiolates. 2. Kinetics and mechanism of dissociation to nitric oxide in aqueous solution. Journal of the American Chemical Society 2001, 123 (23), 5473-5481.

12. Mowery, K.; Meyerhoff, M., The transport of nitric oxide through various polymeric matrices. Polymer 1999, 40 (22), 6203-6207.

13. Moynihan, H. A.; Roberts, S. M., Preparation of some novel S-nitroso compounds as potential slow-release agents of nitric oxide in vivo. J. Chem. Soc., Perkin Trans. 1 1994, (7), 797-805.

14. Tinkilic, N.; Cubuk, O.; Isildak, I., Glucose and urea biosensors based on all solid-state PVC-NH 2 membrane electrodes. Analytica chimica acta 2002, 452 (1), 29-34.

15. Liu, J.; Hsieh, Y. Z.; Wiesler, D.; Novotny, M., Design of 3-(4carboxybenzoyl)-2-quinolinecarboxaldehyde as a reagent for ultrasensitive determination of primary amines by capillary electrophoresis using laser fluorescence detection. Analytical chemistry 1991, 63 (5), 408-412.

16. Starrett, M. A.; Nielsen, M.; Smeenge, D. M.; Romanowicz, G. E.; Frost, M. C., Wireless platform for controlled nitric oxide releasing optical fibers for mediating biological response to implanted devices. Nitric Oxide 2012, 27 (4), 228-234

17. Frost, M. C.; Reynolds, M. M.; Meyerhoff, M. E., Polymers incorporating nitric oxide releasing/generating substances for improved biocompatibility of bloodcontacting medical devices. Biomaterials 2005, 26 (14), 1685-93.

18. Ellman, G. L., Tissue sulfhydryl groups. Archives of biochemistry and biophysics 1959, 82 (1), 70-77. 
19. Kolluru, G. K.; Sinha, S.; Majumder, S.; Muley, A.; Siamwala, J. H.; Gupta, R.; Chatterjee, S., Shear stress promotes nitric oxide production in endothelial cells by sub-cellular delocalization of eNOS: A basis for shear stress mediated angiogenesis. Nitric Oxide 2010, 22 (4), 304-315.

20. Chen, C.; Hanson, S. R.; Keefer, L. K.; Saavedra, J. E.; Davies, K. M.; Hutsell, T. C.; Hughes, J. D.; Ku, D. N.; Lumsden, A. B., Boundary layer infusion of nitric oxide reduces early smooth muscle cell proliferation in the endarterectomized canine artery. Journal of Surgical Research 1997, 67 (1), 2632.

21. Issa, Y. M.; Khorshid, A. F., Using PVC ion-selective electrodes for the potentiometric flow injection analysis of distigmine in its pharmaceutical formulation and biological fluids. Journal of Advanced Research 2011, 2 (1), 25-34.

22. Yoshinaga, T.; Yamaye, M.; Kito, T.; Ichiki, T.; Ogata, M.; Chen, J.; Fujino, H.; Tanimura, T.; Yamanobe, T., Alkaline dechlorination of poly (vinyl chloride) in organic solvents under mild conditions. Polymer degradation and stability 2004, 86 (3), 541-547. 


\section{Chapter 3 : High Capacity Nitric Oxide Release from $S$-Nitroso- $N$-Acetyl-D-Penicillamine Modified Hyperbranched Polyamidoamine (SNAP-HPAMAM) for Controlled Nitric Oxide Release}

\subsection{Introduction}

Nitric oxide (NO) has been proven to be an important molecule with multiple functions such as maintaining vascular health, mediating inflammatory response, and preventing bacterial adhesion depending on the level of NO that is being released ${ }^{1-3}$. To further the advancement of biomedical devices and sensors, being able to mediate and reduce the foreign body response of a patient is an important factor. For patients that are in critical condition where an important analyte is constantly monitored, biosensors must be able accurately measure it. Due to the foreign body response, these medical devices often lose their functionality over a short period of time. NO has the ability to minimize unwanted inflammatory response and increase the longevity of implantable medical devices. The two most popular NO releasing materials to be applied in a medical application are S-nitrosothiols and $N$-diazeniumdiolates ${ }^{4-5}$. These molecules are able to be covalently attached to materials to provide stable, NO releasing compounds. The release mechanisms of these and other NO donors have been well characterized ${ }^{6}$. Long term NO release at a physiological level is necessary for keeping implantable devices free from unwanted immune response, favoring the slower and more controlled release mechanism of S-nitrosothiol chemistry. Using an NO donor with the potential for a high NO reservoir, polymers would able to contain the amount of NO required for these long term situations. ${ }^{2}$

Dendrimers are highly branched, symmetrical macromolecules that can have a wide array of surface chemistry characteristics. They start with a core molecule, and from there can progress in size and functionality with stepwise reactions done in separate

\footnotetext{
${ }^{2}$ Material in this chapter is in the process of being submitted for publication.
} 
batch processes. Several groups of researchers led by Buhlier, Newkome, and Tomalia pioneered the development of dendritic type macromolecules to eventually lead to the large field of dendrimer research that is seen today ${ }^{7-9}$. A similar class of macromolecule to dendrimers are hyperbranched polymers. Hyperbranched polymers do not have the symmetry that is seen with dendrimers, and are usually a large array of randomly sized molecules as there are no purification or chromatography steps during the processing ${ }^{10}$. Because of this, hyperbranched polymers act more like synthetic polymers as they have a large molecular weight distribution of different sized chains, whereas perfectly synthesized dendrimers only contain a single mass to be observed ${ }^{11}$. Dendrimers and hyperbranched molecules have the ability contain a large reservoir of NO due to the large amount of surface functional groups they contain. Dendrimers have been widely used in medicine and are continuing to grow in popularity due to its molecular storage capabilities and high functionality ${ }^{12}$. They are used in a variety of medical applications such as cancer treatments, gene therapy, and precise drug delivery ${ }^{12-15}$. A popular class of dendrimer that is widely used in research is polyamidoamine (PAMAM). PAMAM dendrimer and hyperbranched polymer cytotoxicity has been previously investigated and found that the main mechanism for which these molecules decrease cell viability is through cationic disruption of cell plasma membranes ${ }^{16,17}$. By modifying the primary amine sites with SNAP functional groups, PAMAM dendrimers have been shown to not negatively affect the cells they interacts with ${ }^{18}$.

NO releasing dendrimers have been tested before by Stasko et al. to demonstrate the NO storage capability of this type of molecular architecture ${ }^{19}$. The authors were able to fully characterize 64 armed dendrimers with the ability to deliver large, precise amounts of NO. While this type of NO releasing molecule is excellent for targeted therapeutics, it is limited in its role for integrating into polymer matrices. The synthesis route for creating high generation dendrimers takes a large amount of labor and time due to the extent of the purification processes required. Hyperbranched polymers are similar to dendrimers but contain defects within the structure due to skipping these purification process steps during synthesis ${ }^{20}$. Hyperbranched polyamidoamine (HPAMAM) 
molecules can be synthesized in a simple and economical method as a high yield of product is obtained over a shorter period of time is compared to pure PAMAM dendrimers. The importance of the compound is stressed on the functional groups more than the dendritic structure when looking into high capacity, blendable NO donating materials. The significantly reduced reaction times and purification steps make these highly functionalized hyperbranched polymers attractive for use as an NO delivery vehicle to be blended into various polymeric matrices to develop a range of NO releasing materials, simply by varying the amount of donor blended into the base polymer.

NO releasing hyperbranched polyethers have been previously synthesized using $\mathrm{N}$ diazeniumdiolate NO donors, but have shown poor NO addition efficiency ${ }^{21} . \mathrm{N}$ diazeniumdiolate molecules also do not have the controllability as seen with SNAP based NO donors as its release mechanism is triggered when put in physiological $\mathrm{pH}$ and temperature ${ }^{22}$. The purpose of creating a SNAP-HPAMAM compound is to use to blend within other polymers while still maintaining all of the beneficial properties of a SNAP based NO donor, giving the option of either having a controlled or passive release of NO.

Herein, HPAMAM was modified with the S-nitrosothiol, N-acetyl-D-penicillamine, and nitrosated to form a controlled NO donating compound (SNAP-HPAMAM). This compound was triggered to release NO by photoinitiation and ion mediated release. Structural analysis of a generation 1 HPAMAM molecule was done through FTIR, ${ }^{1} \mathrm{H}$ NMR, and MALDI-TOF mass spectroscopy. The NO capacity of a generation 1 SNAPHPAMAM molecule ended up being approximately $1.90 \mu \mathrm{mol} \mathrm{NO} / \mathrm{mg}$. Quantification of the functional groups in the compound proved that an average of 6.40 reactive primary amine sites per molecule were present compared to the 8 reactive sites on a perfectly synthesized generation 1 dendrimer.

\subsection{Experimental Details}




\subsubsection{Materials}

Ethylenediamine, methyl acrylate, methanol, ethanol, toluene, pyridine, acetic anhydride, chloroform, hexanes, hydrochloric acid, magnesium sulfate, $\alpha$-cyano-4hydroxycinnamic acid, Lugol's iodine, glacial acetic acid, Ellman's reagent were all purchased from Sigma Aldrich (St. Louis, MO, USA). ATTO-TAG FQ reagent was purchased from Invitrogen (Grand Island, NY, USA). Tert-butyl nitrite (90\% technical grade, Acros Organics) was purchased from Fisher Scientific.

\subsubsection{Synthesis of HPAMAM}

Generation 1 HPAMAM molecules were synthesized following a modified procedure previously done ${ }^{23}$. The core molecule to initiate the hyperbranched polymer was 83 mmol of ethylenediamine (EDA), which was dissolved in $250 \mathrm{~mL}$ of methanol and cooled in an ice bath. $340 \mathrm{mmol}$ of methyl acrylate (MA) was added drop wise to the stirring solution of EDA and methanol. The solution was then allowed to stir at room temperature for 48 hours. The methanol and unreacted MA was removed by rotary evaporation at $45^{\circ} \mathrm{C}$ to yield a four armed ester terminated hyperbranched polymer. 742 mmol of EDA was dissolved in $100 \mathrm{~mL}$ of methanol in a separate container and chilled in an ice bath. This solution was then added drop wise to the stirring ester terminated hyperbranched polymer and allowed to stir at room temperature for 72 hours. Rotary evaporation was then done to remove the most of the unreacted EDA and methanol at $45^{\circ} \mathrm{C}$. Excess EDA was removed by adding a toluene and methanol mixture with a ratio of 9:1 (v/v) respectively at its temperature azeotrope to yield a four armed amine terminated molecule (generation 0 HPAMAM).

The same reaction process was then repeated where MA in two times molar excess is dissolved in $100 \mathrm{~mL}$ of methanol and added drop wise to the chilled generation 0 HPAMAM and allowed to stir for 48 hours at room temperature. Repeating the EDA reaction step and removal method results in an eight armed amine terminated molecule (generation 1 HPAMAM). The solution becomes noticeably more viscous and amber in 
color after each step. The reaction schematic and final product formed is shown in

Figure 3.1.
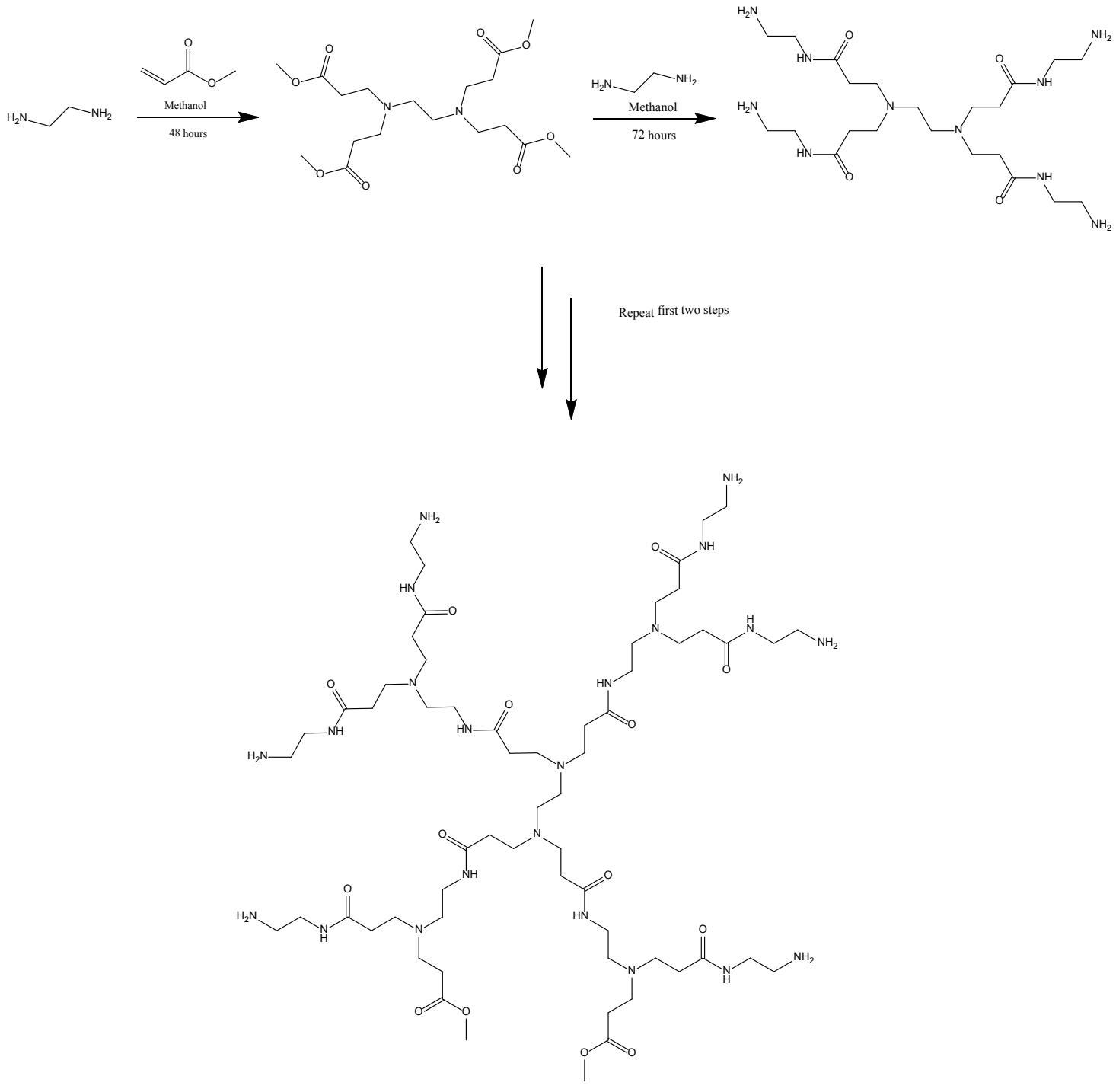

Figure 3.1. Synthesis schematic of hyperbranched polyamidoamine (HPAMAM). Ethylenediamine is used as the core starting molecule, and is then propagated through the Michael addition of methyl acrylate. The process is then repeated until the desired hyperbranched structure is created.

\subsubsection{Synthesis of NAP-thiolactone}


The procedure described in section 2.2.2 was used for the synthesis of NAP-thiolactone and its reaction scheme is seen in Figure 2.1.

\subsubsection{Nitrosation of N-acetyl-D-penicillamine modified HPAMAM}

There are two methods that can be employed to nitrosated HPAMAM. Using acidified nitrites as a nitrosation pathway was done for the first method. The synthesized generation 1 HPAMAM (400 mg) was dissolved in $5 \mathrm{~mL}$ of deionized water. 3.00 mmol $(519 \mathrm{mg})$ of NAP-thiolactone was added to the stirring solution and was allowed to react for 24 hours to form a NAP-HPAMAM compound. An additional $5 \mathrm{~mL}$ of deionized water may need to be added to ensure all of the NAP-thiolactone is dissolved. The NAP-HPAMAM solution that is formed was mixed with an equal volumetric amount of $1 \mathrm{M}$ hydrochloric acid and chilled in ice for one hour. An abundance of sodium nitrite $(230 \mathrm{mg}, 3.33 \mathrm{mmol})$ was then added to the solution and allowed to react at $0^{\circ} \mathrm{C}$ for 45 minutes. As the reaction occurs, the color will change from clear/light yellow to a dark green. The solution was then rotary evaporated at room temperature until most of the solvent is removed. The resulting SNAP-HPAMAM was then dissolved in chilled ethanol and mixed thoroughly. The ethanol solution was filtrated of any excess sodium nitrite or unreacted precipitates using $0.22 \mu \mathrm{m}$ PVDF syringe filters. The SNAP-HPAMAM ethanol solution was rotary evaporated at room temperature until a green viscous precipitate is left. This precipitate was then placed in a vacuum oven at room temperature until the green viscous product is completely dried. The resulting crystalline product can then be recovered and stored. Shielding the compound from light was maintained during all processing steps whenever possible.

The second method uses tert-butyl nitrite for nitrosating the NAP-HPAMAM. Generation 1 HPAMAM (400 mg) is dissolved in $5 \mathrm{~mL}$ of methanol and the same amount of NAP-thiolactone is added as was previously mentioned. The solution was then allowed to react for 24 hours. $1 \mathrm{M} \mathrm{HCl}$ was then added to the NAP-HPAMAM until the $\mathrm{pH}$ of the solution is approximately 5.0. The resulting NAP-HPAMAM methanol solution is then reacted with tert-butyl nitrite in excess $(500 \mu \mathrm{L}, 4.84 \mathrm{mmol})$. Before 
adding the tert-butyl nitrite, it was first cleaned by using $30 \mathrm{mM}$ cyclam $(1,4,8,11$ tetraazacyclotetradecane) to chelate any metal ions present that could cause RSNO degradation. After the addition of the cleaned tert-butyl nitrite to the NAP-HPAMAM, the solution should change from clear/light yellow to a deep green color over the course of an hour. To remove the excess solvent, it was rotary evaporated at $35^{\circ} \mathrm{C}$ and then vacuum dried at room temperature to obtain a crystalline product. For situations where

your compound would need to be kept in the aqueous phase during reacting or blending with other materials, utilizing the acidified sodium nitrite method is preferred. Using tert-butyl nitrite to nitrosate the NAP-HPAMAM is the preferred method for when working with organic solvents. When isolated and dried, the tert-butyl nitrite nitrosated SNAP-HPAMAM looked a much deeper green color when compared to the first method that uses sodium nitrite. This could be due to the less steps of processing that is required, preventing the exhaustion of $\mathrm{NO}$ in the material over time.

\subsubsection{Nitric oxide release}

Nitric oxide released from the compound was directly measured by the chemiluminescent reaction of NO with ozone using a Sievers 280i Nitric Oxide Analyzer (GE Instruments). This was used to determine the variation of NO flux from polymer films containing SNAP-HPAMAM with light and for determining the total NO capacity of SNAP-HPAMAM. SNAP-HPAMAM was cast into films using polyvinyl chloride (PVC) as the base polymer. For determining photoinitiated NO release, the films were placed in a two armed amber glass sample holder $5 \mathrm{~cm}$ above a mounted LED. The LED was used to trigger the NO release at a variety of voltage levels at 130 $\Omega$ to show the controllability of SNAP based NO donors.

NO capacity quantification was achieved by tri-iodide reduction following the procedure from Yang et $\mathrm{al}^{24}$. An $\mathrm{I}_{3}{ }^{-}$solution was first made by creating a $3 \%$ by weight iodine solution and mixing it with acetic acid in a 2:7 ratio by volume respectively. A recorded weight of SNAP-HPAMAM was first pre-treated with a 5\% acidified sulfanilamide solution to react with any unreacted nitrites that could be. After allowing 
the $\mathrm{I}_{3}{ }^{-}$solution to stir for 30 minutes, it was added to the measured SNAP-HPAMAM while being stirred at room temperature.

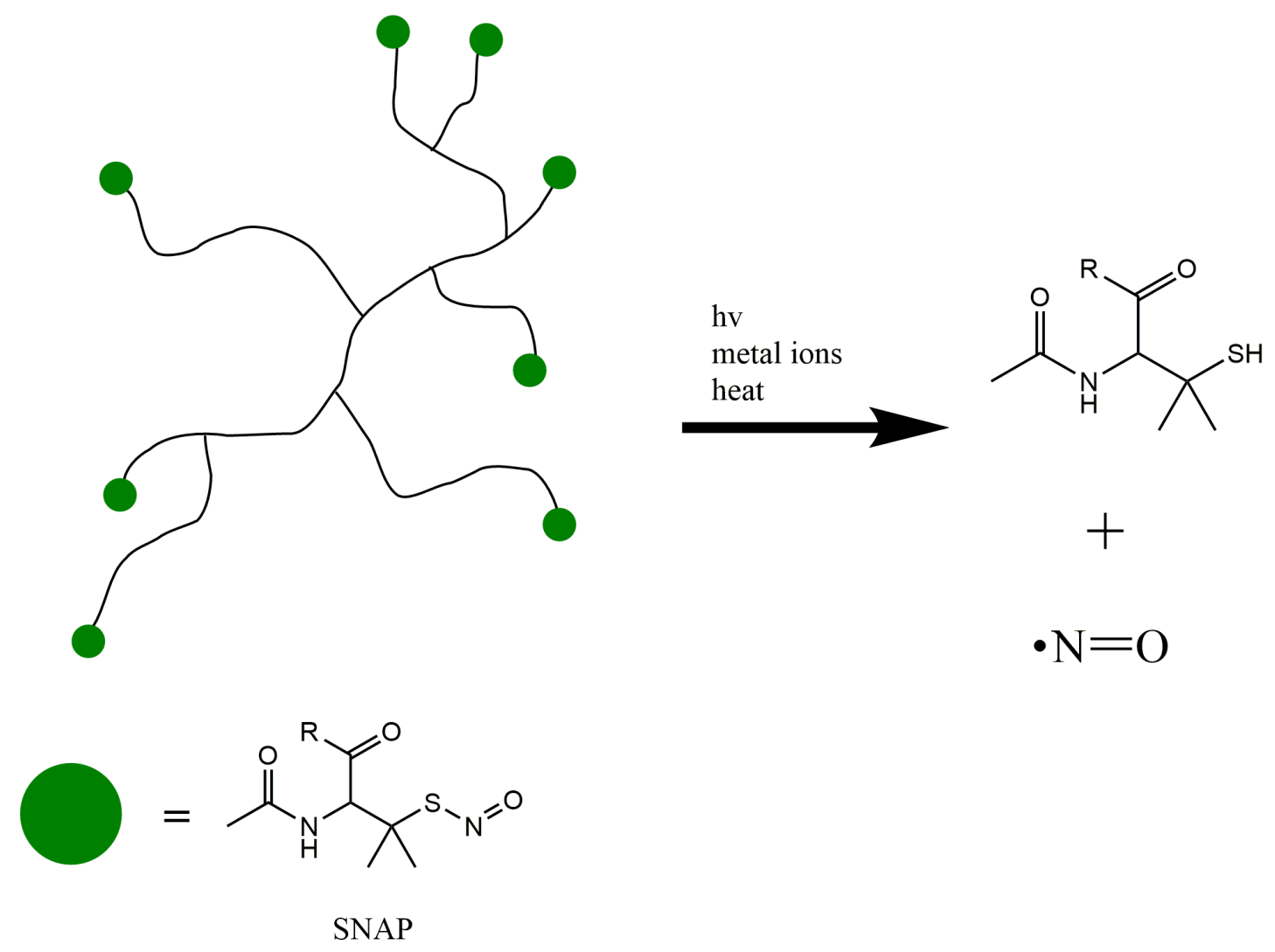

Figure 3.2. Schematic of SNAP-HPAMAM's generic structure and triggered NO release methods. The hyperbranched system shows a disorganized but highly functionalized network capable of storing large amounts of NO by attaching SNAP to the reactive end groups in the HPAMAM structure. The NO can be triggered through light, ion interaction, and heat.

\subsubsection{Material characterization}

FTIR, NMR, and MALDI-TOF mass spectroscopy were done to obtain the general structure of the HPAMAM and NAP-HPAMAM molecules being synthesized. Quantification of primary amines was accomplished using by fluorescently tagging each site with ATTO-TAG FQ. Excitation was at $450 \mathrm{~nm}$ while the emission wavelength was at $550 \mathrm{~nm}$. After each free primary amine site was reacted with NAP-thiolactone, 
Ellman's test for free thiols was done to quantify the conversion of amines to thiols within the hyperbranched structures. Optical absorbance was taken at $412 \mathrm{~nm}$ using a 96-well plate reader. The quantified results were then used to further verify the hypothesized structures obtained from the FTIR and MALDI-TOF data.

\subsection{Results and Discussion}

\subsubsection{Photoinitiated nitric oxide release}

One of the main mechanisms for controlled release of NO from RSNOs is through homolytic cleavage of the sulfur-nitrogen bond via the administration of light. In the dark, the SNAP-HPAMAM material is stable while at room temperature, but can have a controlled and stable release of $\mathrm{NO}$ when different levels of light are introduced. The stability translates to the NO release profiles seen in Figure 3.3, where SNAPHPAMAM was distributed evenly into PVC thin films and tested. The control in NO release is seen by photoinitiated release at various intensities of light using a $470 \mathrm{~nm}$ wavelength LED at $130 \Omega$. Voltages are adjusted to increase or decrease the drive current applied to the LED and the then turned off to demonstrate the time to takes to reach a normal baseline release. Once the peak level of NO release is reached for each LED flux step, there is little to no decay. The benefit of having a controllable NO releasing material is the application for in vitro testing. Different cell types interact with specific levels of $\mathrm{NO}$ in different ways. Most in vitro testing of $\mathrm{NO}$ administration is usually done by adding a solution of $\mathrm{N}$-diazeniumdiolates NO donor directly to cell media. This method only gives a short lived burst of NO before being diminished. NO contact with cells usually comes in the form of a flux, where a constant and steady level of $\mathrm{NO}$ is released over time. Using the light sensitive properties of RSNOs, an in vitro cell culture setup could have a level flux of NO sent to the cells over a long period of time. 


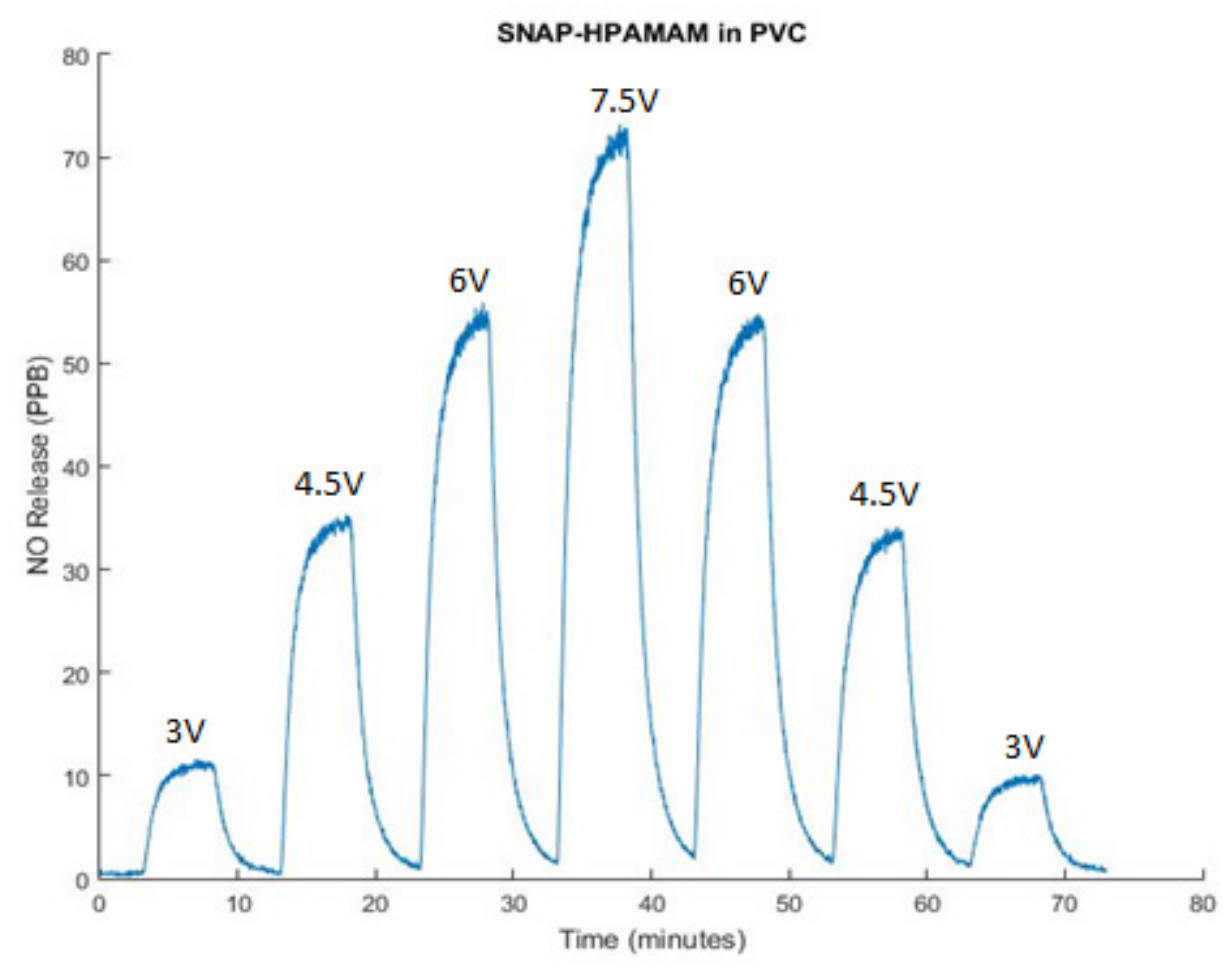

Figure 3.3. NO release profile of SNAP-HPAMAM blended in PVC using photoinitiation. Samples were placed in amber, two armed vials with a nitrogen sweep gas where a $470 \mathrm{~nm}$ LED was mounted $5 \mathrm{~cm}$ above the polymer film being tested. The resistance was held constant at $130 \Omega$ while the voltage was increased and decreased in increments of $1.5 \mathrm{~V}$, starting from $3 \mathrm{~V}$. Before each step increase, the LED was turned off to demonstrate the controllability photoinitiated NO release contains. The films were $5.5 \mathrm{~mm}$ in diameter and $0.1 \mathrm{~mm}$ thick.

\subsubsection{Ion mediated nitric oxide release}

The SNAP-HPAMAM that was used for photoinitiated NO release was also tested for its passive release from ions. SNAP has the ability to release NO from a variety of different ions in solution such as copper, ascorbate, and iron ${ }^{4}$. Using the SNAPHPAMAM that was nitrosated using the acidified sodium nitrite mechanism, multiple ion mediated release pathways were tested. $2.16 \mathrm{mg}$ of SNAP-HPAMAM was placed in $\mathrm{PBS}$ at $37^{\circ} \mathrm{C}$ to give a representative $\mathrm{NO}$ release profile under in vivo conditions and is shown in Figure 3.4. In aqueous conditions, SNAP-HPAMAM tends to swell and 
dissolve readily. The large peak of NO release seen once the PBS is added can be explained by the ubiquitous transition metal ions present in PBS initiating catalytic decomposition of the RSNOs present.

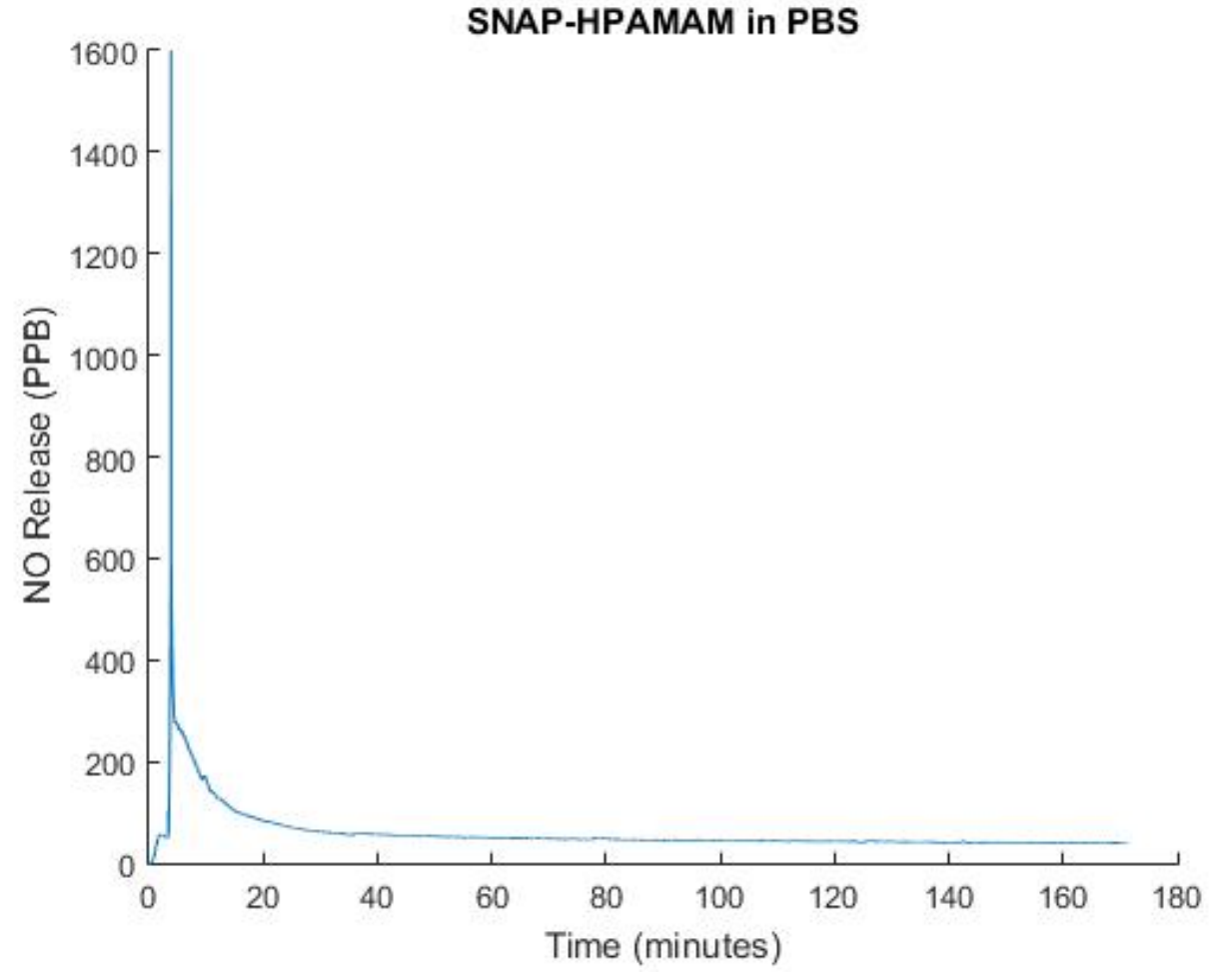

Figure 3.4. Passive NO release from $2.16 \mathrm{mg}$ of SNAP-HPAMAM in $2 \mathrm{~mL}$ of PBS at $37^{\circ} \mathrm{C}$. The material was first placed into a two armed amber reaction vessel and allowed to warm to temperature. PBS was added at $37^{\circ} \mathrm{C}$ to observe how NO is released from SNAP-HPAMAM while under physiological conditions.

SNAP-HPAMAM is stable when blended into films with other hydrophobic polymers and is able to retain the NO donating group for longer periods of time by decreasing the access of transition metal ions to the RSNOs compared to directly administering it into solution. Figure 3.5 shows how this method increases the longevity of the NO donor when $6 \mathrm{mg}$ of SNAP-HPAMAM were blended with a solution of PVC dissolved in N,N-dimethylacetamide and cast into $10 \mathrm{~mm}$ diameter films. If a hydrophilic polymer like polyvinyl alcohol was used instead, it would allow the uptake of water and eventually the release of the immobilized SNAP-HPAMAM 
into the surrounding area as it swells. This would cause problems as the localized NO release would no longer be controllable. To prevent this, PVC was used as the polymer containing SNAP-HPAMAM due to its properties as a membrane for ion-selective electrodes ${ }^{25}$. Other hydrophobic polymers like polydimethylsiloxane (PDMS) further prevent ion diffusion into the polymer compared to PVC, and would be suitable for situations where only thermal degradation of the RSNO is required. PVC gives the option of using ion mediated NO release of the SNAP functional group while still having the option of a more controlled release through a photolytic response. A characteristic seen with blending SNAP-HPAMAM within PVC is its abilty to act like a plasticizer. Unplasticized PVC tends to be quite brittle but still manageable when cast into thin films. SNAP-HPAMAM PVC is less brittle than pure PVC, and has more elastic properties when the concentration within the polymer matrix is high enough. Hyperbranched polyesters have been known to be suitable, safe plasticizers in $\mathrm{PVC}^{26}$. The unreacted, defected ester branches see in HPAMAM are most likely providing a similar plasticizer property seen in the hyperbranched polyester blends. 


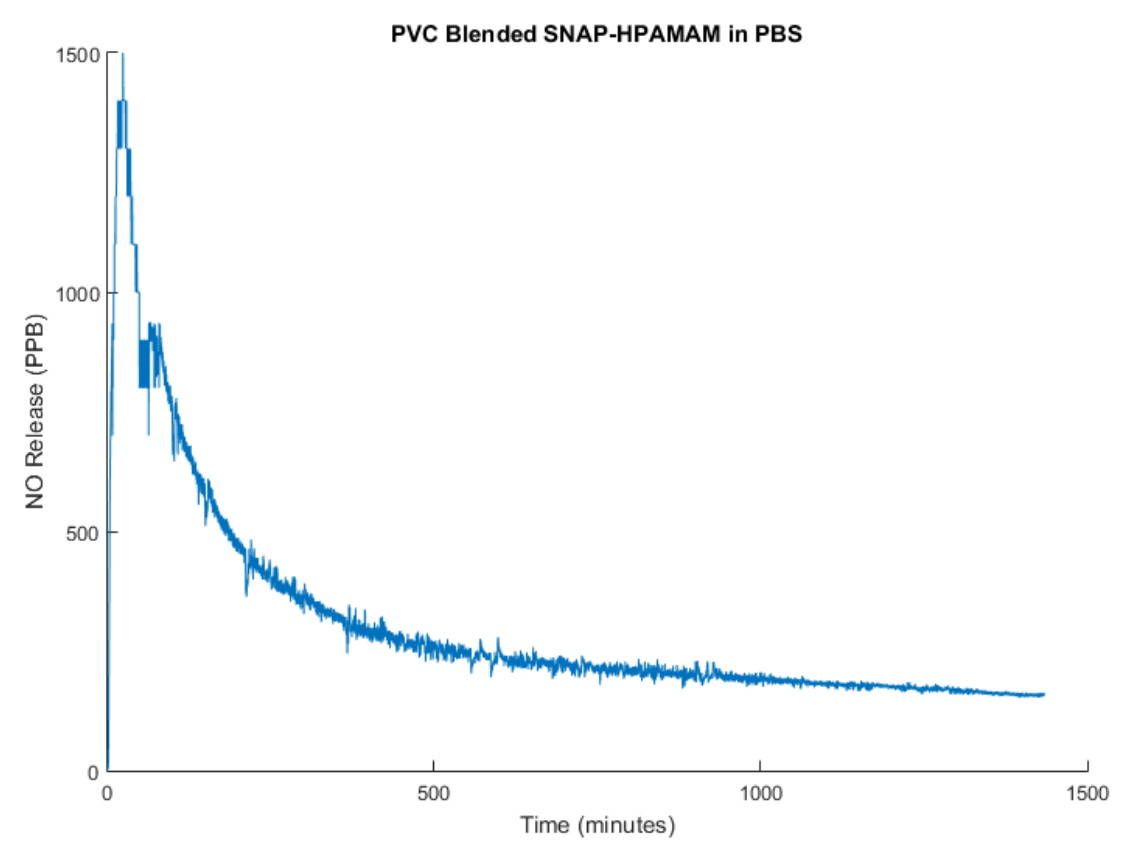

Figure 3.5. Passive NO release of $6.01 \mathrm{mg}$ of SNAP-HPAMAM encapsulated in PVC in PBS at $37^{\circ} \mathrm{C}$. When compared to Figure 3.4, the encapsulation of the material shows an increase in longevity as a high release of $\mathrm{NO}$ is able to be sustained over a longer period of time. Preventing the NO donor from leaching out of the polymer matrix allows for very long term, sustained release dependent on the amount of material loaded within the polymer.

Other hydrophobic polymers can be used as well to give off different release profiles of SNAP-HPAMAM while in PBS. To demonstrate this versatility, multiple polymers were blended with the same amount of SNAP-HPAMAM and then placed under physiological conditions. This comparison between the polymers is shown in Figure 3.5. The ion diffusion capability of $\mathrm{PVC}$ is proven here as it demonstrates the highest amount of NO release. Other materials such as RTV-3140 do not show as high of a release, but are able to release at a steady flux over a long period of time. 


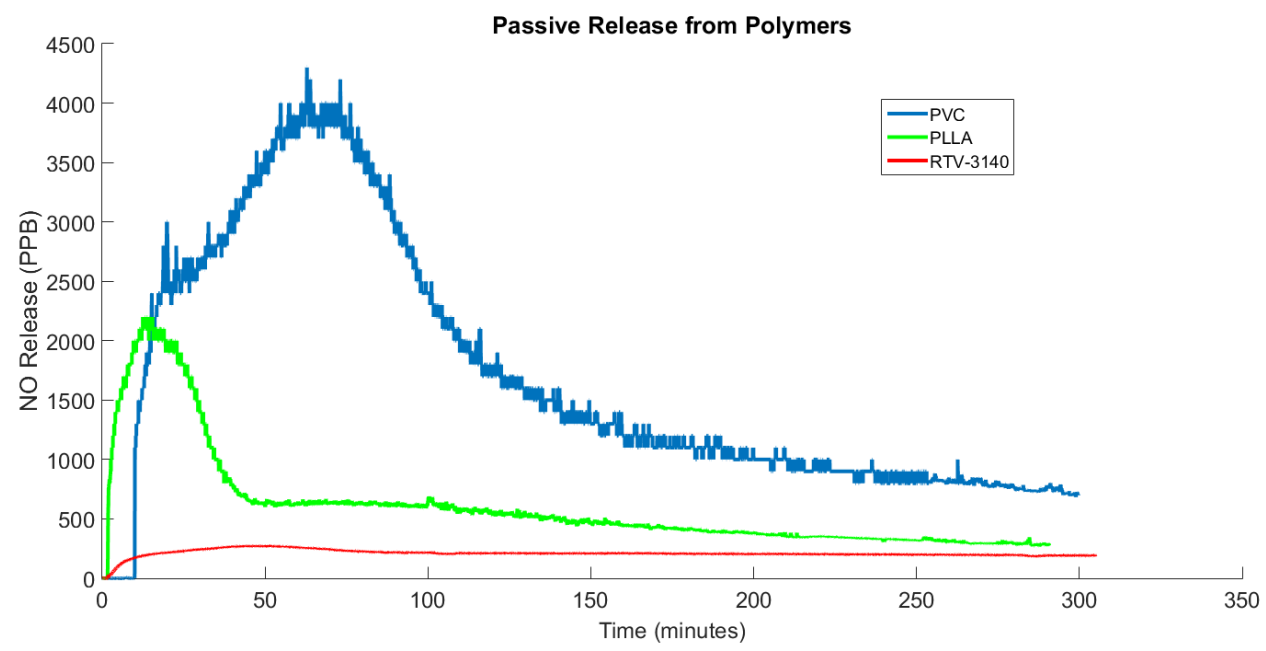

Figure 3.6. Passive $\mathrm{NO}$ release in $\mathrm{PBS}$ at $37^{\circ} \mathrm{C}$ from multiple polymers blended with SNAP-HPAMAM. $10 \mathrm{mg}$ of SNAP-HPAMAM was blended into multiple films (15 $\mathrm{mm}$ diameter). This shows the flexibility of SNAP-HPAMAM blended hydrophobic polymeric systems as the amount of NO over time can be adjusted based on the polymer properties it is immobilized in. PVC (orange) showed the highest passive release when placed in PBS along with a decent amount of release seen in PLLA (blue). SNAPHPAMAM blended in RTV-3140 (gray) showed the lowest amount of release due to its ability to prevent ion diffusion, but demonstrates a very steady release profile which can still be useful in specific situations.

\subsubsection{FTIR analysis}

Identification of the important functional groups of the HPAMAM compound was seen in the FTIR data collected seen in Figure 3.7. The presence of primary amines seen in the HPAMAM spectra is seen around the $3300 \mathrm{~cm}^{-1}$ range (N-H stretching) and 1620 $\mathrm{cm}^{-1}$ (N-H bending), which are then removed once the attachment of NAP-thiolactone is added, which reacts with primary amine groups. The important note of transition from NAP-HPAMAM to SNAP-HPAMAM is the disappearance of the thiol peak at 2550 $\mathrm{cm}^{-1}$ once it is nitrosated, showing an efficient addition of the NO group to the compound. The NO peak is difficult to see due to the presence of the large amount of amides in the structure, which contain carbonyl groups at roughly $1500 \mathrm{~cm}^{-1}$. Nitroso peaks are also commonly seen in this range. 


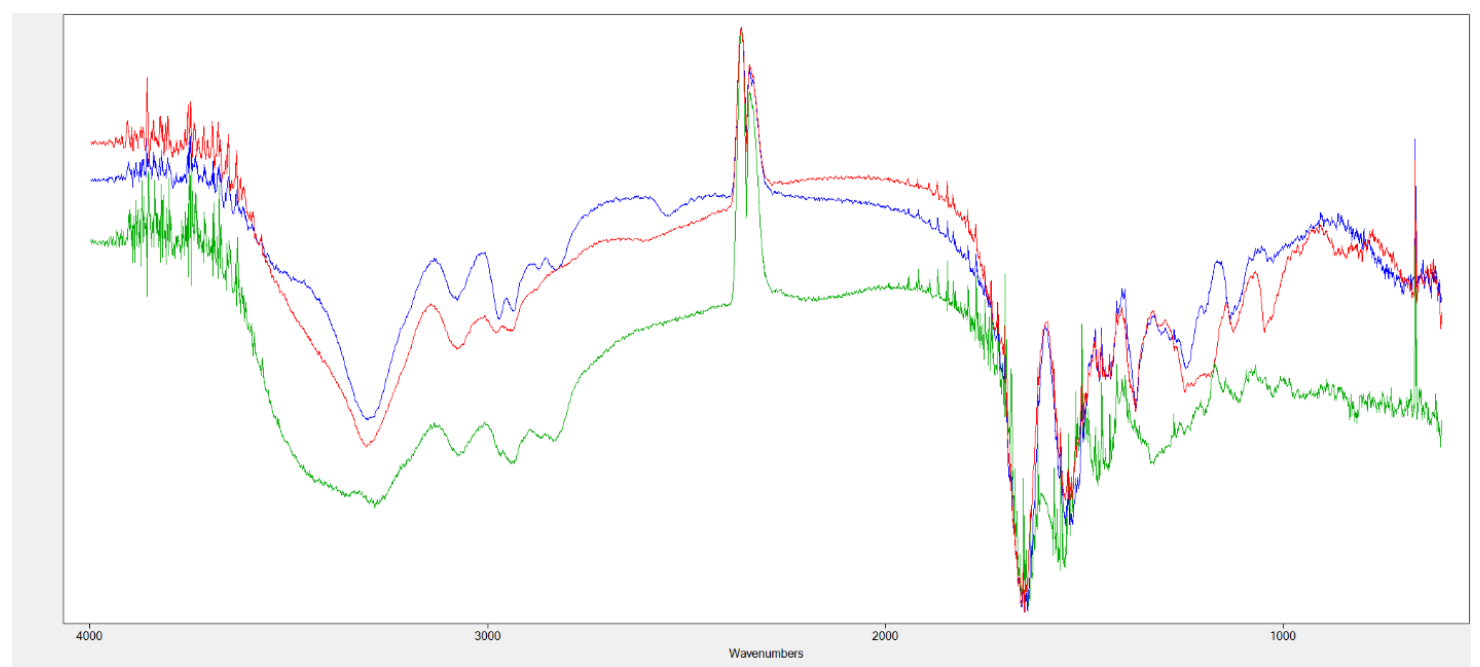

Figure 3.7. FTIR spectrum of hyperbranched compounds. Demonstrates the progression of HPAMAM (green), NAP-HPAMAM (blue), and SNAP-HPAMAM (red) compounds. The N-H stretches from primary amines are visible around the 3300-3400 $\mathrm{cm}^{-1}$ range for HPAMAM which then disappears when NAP-thiolactone is attached. Once NAP-HPAMAM is formed, a free thiol peak is seen at 2550. This thiol is then nitrosated as the peak is no longer present when it is nitrosated to form SNAPHPAMAM.

\subsubsection{NMR analysis}

NMR was also done on the base HPAMAM compound to verify the initial synthesis procedure is producing the correct dendritic structure. The important characteristic functional groups seen in PAMAM dendrimers were classified within the NMR spectrum. ${ }^{1} \mathrm{H}$ NMR (400 MHz, $\left.\mathrm{CDCl}_{3}\right): \delta 7.47\left(7 \mathrm{H}, \mathrm{CONHCH}_{2}, \mathrm{t}\right), 3.41(4 \mathrm{H}, \mathrm{COOCH}$, s) $3.23\left(14 \mathrm{H}, \mathrm{NHCH}_{2} \mathrm{CH}_{2}, q\right), 2.70\left(13 \mathrm{H}, \mathrm{CH}_{3} \mathrm{CH}_{2} \mathrm{~N}, \mathrm{t}\right), 2.63\left(11 \mathrm{H}, \mathrm{CH}_{2} \mathrm{CH}_{2} \mathrm{NH}_{2}\right.$, quin), $2.31\left(19 \mathrm{H}, \mathrm{CH}_{2} \mathrm{CH}_{2} \mathrm{CO}, \mathrm{t}\right), 1.36\left(22 \mathrm{H}, \mathrm{CH}_{2} \mathrm{NH}_{2}, \mathrm{~s}\right)$. The presence of unreacted ester groups and non-amide containing branches are present, but the primary amine groups remain abundant in the overall hyperbranched structure.

\subsubsection{Quantification of thiols and primary amines}

Quantification of the important functional groups was done by attaching the fluorescent tag ATTO-TAG FQ (3-(2-furoyl) quinoline-2-carboxaldehyde) to the primary amine 
sites present on the HPAMAM molecule following the procedure developed by Liu et al. ${ }^{27}$. The amount of free primary amines present in the compound was calculated to be $4.48 \pm 0.22 \mu \mathrm{mol} / \mathrm{mg}$, which gives an average of $6.40 \pm 0.31$ amines per HPAMAM molecule. A perfectly synthesized generation 1 PAMAM dendrimer would contain 8 primary amines per molecule. Using this hyperbranched synthesis route, there was proven to be not a large loss of amine functionality in the compound.

Ellman's test for free thiols was then used to quantify the amount of thiols present after attaching the synthesized NAP-thiolactone compound to the free primary amines following a modified protocol developed by George Ellman ${ }^{28}$. PBS ( $\left.\mathrm{pH}=7.4\right)$ was used in place of Tris-buffer for the procedure as any excess unreacted NAP-thiolactone can react with the primary amine functional groups present in the Tris-buffer. The amount of free thiols found using the assay was found to be $2.74 \pm 0.30 \mu \mathrm{mol} / \mathrm{mg}$.

\subsubsection{Nitric oxide capacity testing}

Total NO capacity of SNAP-HPAMAM was done through tri-iodide reduction and was compared to the amine and thiol quantification values. A perfectly synthesized 8 armed SNAP derivatized PAMAM dendrimer has the capability to release $5.594 \mu \mathrm{mol}$ of NO per $\mathrm{mg}$ if every primary amine functional group was modified to a SNAP functional group. Hyperbranched polymers contain defects within the structure, so the amount of NO release will be somewhat lower. Since the average amount of free amines are known from the FQ test, the theoretical max conversion of primary amine sites to SNAP should be similar. The synthesized SNAP-HPAMAM demonstrated a capacity of $1.90 \pm 0.12 \mu \mathrm{mol}$ of $\mathrm{NO} / \mathrm{mg}$. Comparing the amount of NO released with the theoretical max gives a $69.4 \%$ molar conversion of NAP-HPAMAM to SNAP-HPAMAM, but only a $42.4 \%$ molar conversion of HPAMAM to SNAP-HPAMAM. Compared to the SNAP-PVC previously described which only had a capacity of approximately 0.0392 $\mu \mathrm{mol}$ of $\mathrm{NO} / \mathrm{mg}$, this is a significant increase in loading potential. The conversion percentage is low most likely due to the processing steps for isolating the material. 
Attaching the NAP-thiolactone to all of the free primary amine arms is quite difficult in a hyperbranched network as there is an abundant of steric hindrance present. During the nitrosation and isolation of SNAP-HPAMAM, the vacuum drying and rotary evaporating steps can take a lot of time. Keeping SNAP-HPAMAM in solution during these steps could be causing passive release, lowering the NO capacity of the final solid-phase product in the end.

\subsubsection{MALDI-TOF analysis}

Figure 3.8 and Figure 3.9 show the MALDI-TOF analysis done on the synthesized HPAMAM and NAP-HPAMAM compounds respectively. $\alpha$-Cyano-4-hydroxycinnamic acid (CHCA) was the matrix used for all tests. Since MALDI-TOF is a softer ionization mass spectroscopy technique, there is no fragmentation occurring during testing. This means that the entire range of peaks seen is a specific hyperbranched molecule with its own branching chemistry.

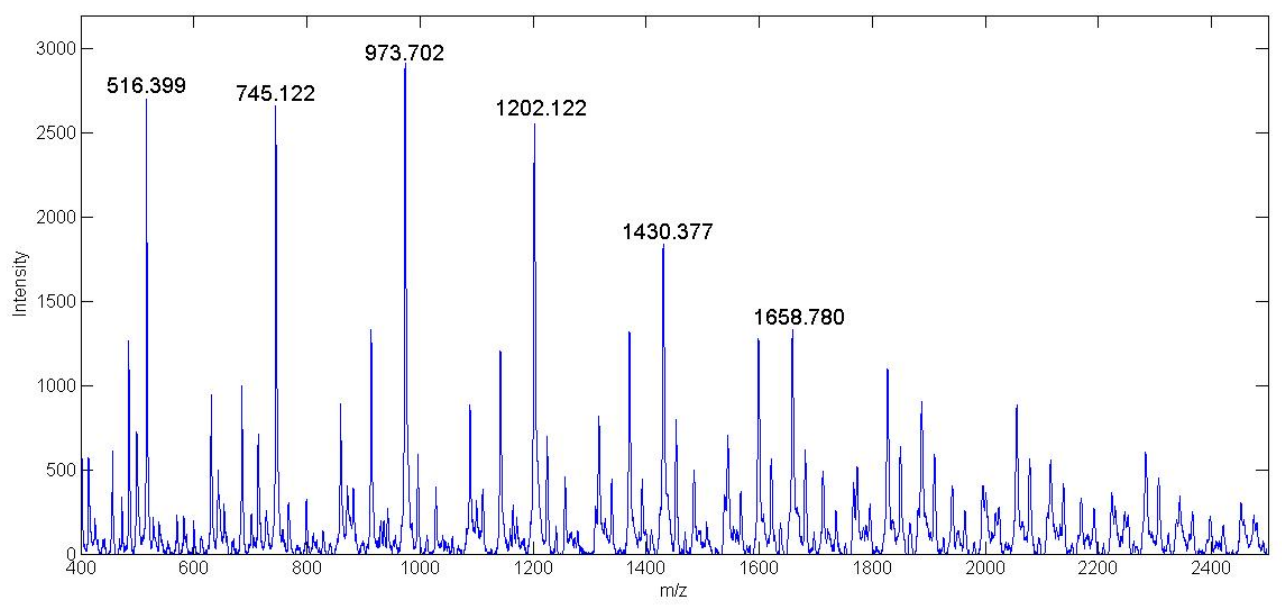

Figure 3.8. MALDI-TOF analysis of HPAMAM. Each labeled peak signifies the addition of a $229 \mathrm{Da}$ amine containing branch to the polymer, where a 4 armed HPAMAM molecule is at $516 \mathrm{Da}, 5$ armed at 745, etc. This gives an insight into the distribution of hyperbranched molecules within the synthesized HPAMAM batch. 


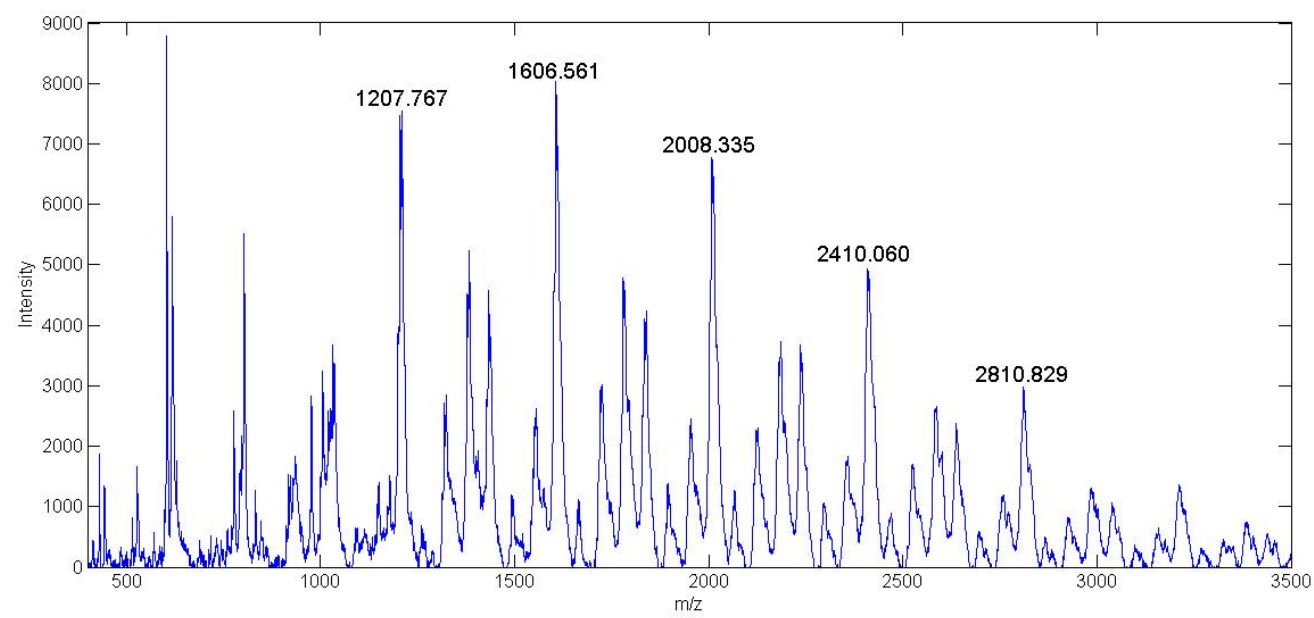

Figure 3.9. MALDI-TOF analysis of NAP-HPAMAM. Each labeled peak signifies the addition of a NAP containing branch site on the polymer, where a 4 armed NAPHPAMAM is at $1207 \mathrm{Da}, 5$ armed at $1606 \mathrm{Da}$, etc. The $229 \mathrm{Da}$ amine containing branches have an extra $173 \mathrm{Da}$ added to them, increasing addition of a reactive arm to approximately $402 \mathrm{Da}$.

A wide range of mass peaks was seen as there are unreacted components still present as the synthesis process of the HPAMAM progresses. The spectra of the materials are characteristic of polymers, where each increment signifies a repeat unit. The spectrum of HPAMAM shows the molecular weight of multiple different branched polymers. The 4 armed HPAMAM starts at a mass of $516 \mathrm{Da}$ and is increased by increments of $229 \mathrm{Da}$ with the addition of another branched functional group. Mass peaks past $1430 \mathrm{Da}$, which is the 8 armed HPAMAM, are most likely due to HPAMAM molecules attaching to each other. The second spectrum shows the attachment of NAP-thiolactone to free primary amine site branches in HPAMAM. The molecular weight of NAP-thiolactone is 173.23 Da, so the 229 Da mass increments between peaks seen in figure 6 are increased to approximately $402 \mathrm{Da}$ for each additional HPAMAM branch. An example of one of the NAP branched hyperbranched structures is shown in Figure 3.10. Each peak seen on both MALDI-TOF spectra can be used to construct some variation of the modified HPAMAM structure. The peak molecular weight of the HPAMAM spectrum is seen at the 973 Da mark, which is the weight of a 6 armed PAMAM structure. This is also 
confirmed by the ATTO-TAG FQ results which gave an estimated 6.40 primary amine sites per HPAMAM molecule.

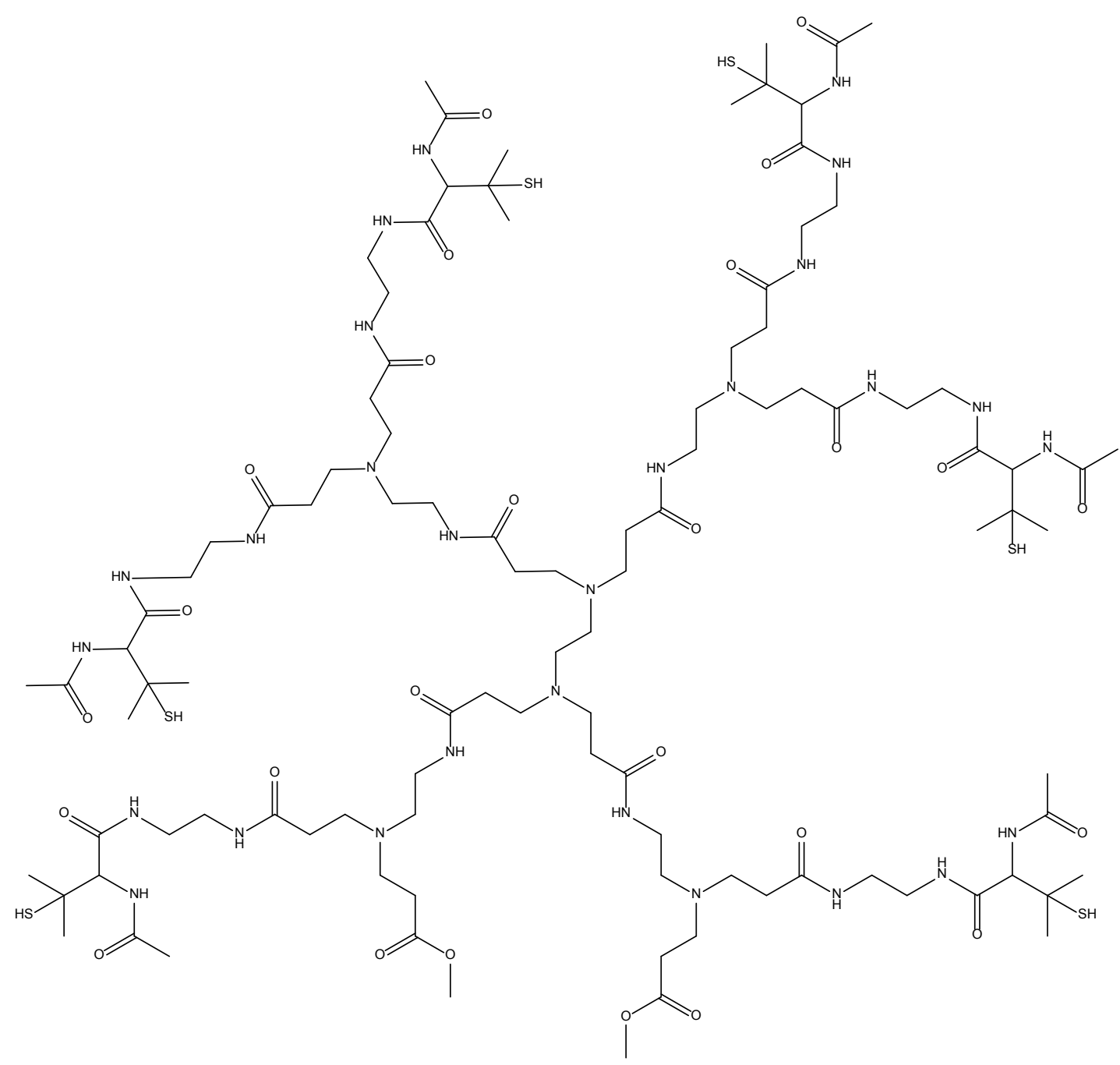

Figure 3.10. Structure of 6 armed NAP-HPAMAM $\left(M_{\mathrm{w}}=2413.14 \mathrm{Da}\right)$. This specific molecular weight from MALDI-TOF is seen in Figure 3.7 and represents the 6 armed NAP branched HPAMAM. Similar structures can be constructed for each peak represented in the MALDI-TOF spectra.

\subsection{Conclusion}


SNAP-HPAMAM was able to show excellent stability and controllability when releasing NO. The synthesis process is shown to be much less tedious than that of dendrimers while still maintaining a relatively high functionality. A large NO capacity of $1.90 \mu \mathrm{mol} / \mathrm{mg}$ was demonstrated, allowing SNAP-HPAMAM to modify a variety of polymers to be high capacity $\mathrm{NO}$ donating biomaterials by simply dispersing it within a polymer matrix. These high capacity NO releasing polymers can then be applied to implantable devices and sensors to increase their longevity while measuring crucial analytes in vivo.

\subsection{References}

1. Chaux, A.; Ruan, X. M.; Fishbein, M. C.; Ouyang, Y.; Kaul, S.; Pass, J. A.; Matloff, J. M., Perivascular delivery of a nitric oxide donor inhibits neointimal hyperplasia in vein grafts implanted in the arterial circulation. The Journal of thoracic and cardiovascular surgery 1998, 115 (3), 604-614.

2. Gifford, R.; Batchelor, M. M.; Lee, Y.; Gokulrangan, G.; Meyerhoff, M. E.; Wilson, G. S., Mediation ofin vivo glucose sensor inflammatory response via nitric oxide release. Journal of Biomedical Materials Research Part A 2005, 75 (4), 755-766.

3. Fang, F. C., Perspectives series: host/pathogen interactions. Mechanisms of nitric oxide-related antimicrobial activity. Journal of Clinical Investigation 1997, 99 (12), 2818.

4. Williams, D. L. H., The chemistry of S-nitrosothiols. Accounts of chemical research 1999, 32 (10), 869-876.

5. Keefer, L. K.; Nims, R. W.; Davies, K. M.; Wink, D. A., "NONOates"(1substituted diazen-1-ium-1, 2-diolates) as nitric oxide donors: convenient nitric oxide dosage forms. Methods in enzymology 1996, 268, 281-293.

6. Wang, P. G.; Xian, M.; Tang, X.; Wu, X.; Wen, Z.; Cai, T.; Janczuk, A. J., Nitric oxide donors: chemical activities and biological applications. Chemical Reviews 2002, 102 (4), 1091-1134. 
7. Buhleier, E.; Wehner, W.; Vögtle, F., " Cascade"-and" Nonskid-Chain-like" Syntheses of Molecular Cavity Topologies. 1978.

8. Newkome, G. R.; Yao, Z.; Baker, G. R.; Gupta, V. K., Micelles. Part 1. Cascade molecules: a new approach to micelles. A [27]-arborol. The Journal of Organic Chemistry 1985, 50 (11), 2003-2004.

9. Tomalia, D. A.; Baker, H.; Dewald, J.; Hall, M.; Kallos, G.; Martin, S.; Roeck, J.; Ryder, J.; Smith, P., A new class of polymers: starburst-dendritic macromolecules. Polymer Journal 1985, 17 (1), 117-132.

10. Gao, C.; Yan, D., Hyperbranched polymers: from synthesis to applications. Progress in Polymer Science 2004, 29 (3), 183-275.

11. Voit, B., New developments in hyperbranched polymers. Journal of Polymer Science Part A: Polymer Chemistry 2000, 38 (14), 2505-2525.

12. Gillies, E. R.; Frechet, J. M., Dendrimers and dendritic polymers in drug delivery. Drug discovery today 2005, 10 (1), 35-43.

13. Majoros, I. J.; Myc, A.; Thomas, T.; Mehta, C. B.; Baker, J. R., PAMAM dendrimer-based multifunctional conjugate for cancer therapy: synthesis, characterization, and functionality. Biomacromolecules 2006, 7 (2), 572-579.

14. Han, S.-o.; Mahato, R. I.; Sung, Y. K.; Kim, S. W., Development of biomaterials for gene therapy. Molecular Therapy 2000, 2 (4), 302-317.

15. Kukowska-Latallo, J. F.; Bielinska, A. U.; Johnson, J.; Spindler, R.; Tomalia, D. A.; Baker, J. R., Efficient transfer of genetic material into mammalian cells using Starburst polyamidoamine dendrimers. Proceedings of the National Academy of Sciences 1996, 93 (10), 4897-4902.

16. Duncan, R.; Izzo, L., Dendrimer biocompatibility and toxicity. Advanced drug delivery reviews 2005, 57 (15), 2215-2237.

17. Nyitrai, G.; Keszthelyi, T.; Bóta, A.; Simon, Á.; Tőke, O.; Horváth, G.; Pál, I.; Kardos, J.; Héja, L., Sodium selective ion channel formation in living cell membranes by polyamidoamine dendrimer. Biochimica et Biophysica Acta (BBA)-Biomembranes 2013, 1828 (8), 1873-1880. 
18. Johnson, T. A.; Stasko, N. A.; Matthews, J. L.; Cascio, W. E.; Holmuhamedov, E. L.; Johnson, C. B.; Schoenfisch, M. H., Reduced ischemia/reperfusion injury via glutathione-initiated nitric oxide-releasing dendrimers. Nitric Oxide 2010, 22 (1), 30-36.

19. Stasko, N. A.; Fischer, T. H.; Schoenfisch, M. H., S-nitrosothiol-modified dendrimers as nitric oxide delivery vehicles. Biomacromolecules 2008, 9 (3), 834-841.

20. Frechet, J. M.; Henmi, M.; Gitsov, I.; Aoshima, S.; Leduc, M. R.; Grubbs, R. B., Self-condensing vinyl polymerization: an approach to dendritic materials. SCIENCE-NEW YORK THEN WASHINGTON- 1995, 1080-1080.

21. Kou, Y.; Wan, A., Synthesis of novel N-diazeniumdiolates based on hyperbranched polyethers. Bioorganic \& medicinal chemistry letters 2008, 18 (7), 2337-2341.

22. Keefer, L. K.; Flippen-Anderson, J. L.; George, C.; Shanklin, A. P.; Dunams, T. M.; Christodoulou, D.; Saavedra, J. E.; Sagan, E. S.; Bohle, D. S., Chemistry of the Diazeniumdiolates I. Structural and Spectral Characteristics of the [N (O) NO]- Functional Group. Nitric Oxide 2001, 5 (4), 377-394.

23. Klaykruayat, B.; Siralertmukul, K.; Srikulkit, K., Chemical modification of chitosan with cationic hyperbranched dendritic polyamidoamine and its antimicrobial activity on cotton fabric. Carbohydrate Polymers 2010, 80 (1), 197-207.

24. Yang, B. K.; Vivas, E. X.; Reiter, C. D.; Gladwin, M. T., Methodologies for the sensitive and specific measurement of S-nitrosothiols, iron-nitrosyls, and nitrite in biological samples. Free radical research 2003, 37 (1), 1-10.

25. Mikhelson, K., Ion-selective electrodes in PVC matrix. Sensors and Actuators B: Chemical 1994, 18 (1), 31-37.

26. Lindström, A.; Hakkarainen, M., Environmentally friendly plasticizers for poly (vinyl chloride) - Improved mechanical properties and compatibility by using branched poly (butylene adipate) as a polymeric plasticizer. Journal of applied polymer science 2006, 100 (3), 2180-2188. 
27. Liu, J.; Hsieh, Y. Z.; Wiesler, D.; Novotny, M., Design of 3-(4carboxybenzoyl)-2-quinolinecarboxaldehyde as a reagent for ultrasensitive determination of primary amines by capillary electrophoresis using laser fluorescence detection. Analytical chemistry 1991, 63 (5), 408-412.

28. Ellman, G. L., Tissue sulfhydryl groups. Archives of biochemistry and biophysics 1959, 82 (1), 70-77. 


\section{Chapter 4 : Subcutaneous Inflammatory Response to Polyvinyl Chloride Based Nitric Oxide Releasing Materials}

\subsection{Introduction}

Implantable medical devices can be of vast importance when patients in critical care require constant monitoring of important analytes like glucose or oxygen. These long term implanted devices lose their functionality over time due to the foreign body response. Depending where the device is implanted, whether it is in contact with blood or tissue, the body will begin to reject and isolate it ${ }^{1}$. For blood contacting devices, proteins will immediately adhere to the surface. This then allows platelets to attach to the device interface and become activated. Once platelet activation occurs, the formation of fibrin leads to a fibrous clot, which has a risk of breaking off and causing an embolism.

Tissue contacting devices have a different foreign body response. In the example of subcutaneous implants, mast cells will first degranulate and release histamine and other cytokines to allow the migration of neutrophils and blood monocytes to the $\operatorname{area}^{2}$. This response is fairly quick, and is done within minutes-hours of the initial implantation. The migrated monocytes then differentiate into macrophages to attempt to phagocytize the foreign material and bacteria that may be present. If the wound healing is not resolved, frustrated phagocytosis occurs as large implanted biomaterials cannot be properly phagocytized compared to bacteria. Eventually the macrophages begin to fuse together to form foreign body giant cells (FBGCs). FBGCs release harsh degradative enzymes and reactive oxygen species as they continue to $a t^{3}$ tempt to break down the implanted biomaterial ${ }^{3}$. This can be problematic for materials with delicate surface chemistry as a large drop in $\mathrm{pH}$ at the FBGC-material interface is seen ${ }^{4}$. Fibrous encapsulation will then begin after FBGCs are unable to phagocytize the foreign

\footnotetext{
${ }^{3}$ Material in this chapter is in the process of being submitted for publication.
} 
material, where a collagenous capsule is formed in an attempt to isolate it off from the rest of the surrounding tissue ${ }^{5}$. For an implanted sensor, this encapsulation causes incorrect readings in the monitored analyte. The foreign body response to implanted biopolymers shows poor biocompatibility when in contact with tissue or blood. Utilizing the anti-inflammatory properties of $\mathrm{NO}$, this unwanted response from the body can be mediated and moved to the resolution phase of wound healing more rapidly.

NO is a free radical signaling molecule produced in the body with a wide range of important functions like smooth muscle relaxation, thromboresistivity, and as an antibacterial agent ${ }^{6-9}$. Macrophages are known to produce NO through inducible nitric oxide synthase (iNOS) as the mechanism for killing foreign bacteria ${ }^{10}$. It is also an important molecule for mediating and controlling the inflammatory response ${ }^{11}$. Using these properties, NO releasing materials have been developed to help prevent unwanted inflammatory responses. A popular NO donor that is able to release its NO reservoir under physiological conditions are N-diazeniumdiolates (NONOates) ${ }^{12}$. This type of NO donor is convenient when administering a short, but precise amount of NO when implanted. Subcutaneous implanted xerogels doped with NONOates have been previously reported to lower the chronic inflammatory response along with reducing the fibrous encapsulation thickness over a 6 week period around an implant ${ }^{13}$. This demonstrates how even a short term administration of NO can have long term and beneficial effects. Subcutaneous sensors that measure glucose are critically important for diabetic patients, but the in vivo longevity is shortened by the foreign body response $^{14}$. The NO donor to be used for this study is S-nitroso-N-acetyl-Dpenicillamine (SNAP), an S-nitrosothiol capable of releasing controlled amounts of NO when in contact with light, specific metal ions, and heat. The passive release seen from SNAP donors are much lower than NONOates when placed under physiological conditions, but in turn are able to release for longer periods of time.

Two different types of NO releasing SNAP based PVC materials were implanted subcutaneously for 1 and 15 days to observe NO's effect on the initial stages of inflammation and its transition into a chronic inflammatory state. The first PVC 
polymer covalently links an NO donating material to the backbone of the polymer (SNAP-PVC) while the second PVC polymer blends a nitrosated hyperbranched polyamidoamine NO donor within the polymer matrix (SNAP-HPAMAM). Each material gives its own distinct NO release profile when under physiological conditions to observe how the inflammatory and wound healing responses are effected by the presence of different administrations of NO.

The inflammatory responses that were investigated were the quantity and phenotype of macrophages present, total cell count, mast cell degranulation, and the fibrous encapsulation around the implant area. The two macrophage phenotypes that were observed were M1 (classically activated) and M2 (alternatively activated) macrophages. M1 macrophages encourage chronic inflammation to a foreign substance while M2 macrophages decreases the inflammatory response while encouraging tissue repair and remodeling ${ }^{15-16}$. Counting the number of macrophages present does not give an accurate representation of the state of inflammation of an implanted material. Observing the ratio of M1 to M2 macrophages that are around the implant site would be able to demonstrate if NO is able to resolve chronic inflammation around an implant. This would then be able to give more insight into how the administration of certain levels of NO can be utilized to benefit long term implanted biomedical devices.

\subsection{Experimental Details}

\subsubsection{Materials}

Methanol, ethanol, methyl acrylate, ethylenediamine, polyvinyl chloride (average $\mathrm{M}_{\mathrm{w}}=$ $\left.233,000, M_{n}=99,000\right), 5,5$ '-dithiobis (2-nitrobenzoic acid), triethylamine, N,Ndimethylacetamide, goat serum, Lugol's iodine solution, 1,4,8,11tetraazacyclotetradecane, N-acetyl-D-penicillamine (Fluka), Polyfreeze medium, bovine serum albumin in PBS, and concentrated hydrochloric acid were obtained from SigmaAldrich (St. Louis, MO, USA). Tert-butyl nitrite (90\% technical grade, Acros Organics) was purchased from Fisher Scientific. Magnesium sulfate, copper (II) bromide, L- 
ascorbic acid sodium salt, and acetic anhydride (Alfa Aesar) were purchased from VWR (West Chester, PA, USA).

For the antibodies, anti-CD11b mouse monoclonal antibody, biotinylated goat anti mouse IgG, endogenous avidin/biotin blocking kit, and Alex Fluor 633 streptavidin was purchased from Abcam (Cambridge, MA, USA). Anti-CD163 mouse monoclonal antibody was purchased from Santa Cruz Biotech (Dallas, TX, USA). iNOS rabbit polyclonal antibody (PA3-030A), ATTO-TAG ${ }^{\mathrm{TM}} \mathrm{FQ}$, and goat anti-Rabbit Alexa Fluor 488 (A-11034) were purchased from Thermo Fisher Scientific (Grand Island, NY, USA).

Staining reagents included Eosin Y disodium salt, acetic acid (99.7\%), Toluidine Blue (powdered), phosphotungstic acid solution 10\% w/v, phosphomolybdic acid hydrate solution, Biebrich scarlet acid-fuchsin solution, Analine blue solution, absolute ethanol, xylene substitute, Gill No. 3 hematoxylin solution, Hematoxylin (powdered), anhydrous Iron (III) chloride (powder 99.99\%), Lugol's iodine Solution, and Eukitt quickhardening mounting medium; all were obtained from Sigma Aldrich.

\subsubsection{Synthesis of self-protected N-acetyl-D-penicillamine (NAP) thiolactone}

The synthesis procedure of NAP-thiolactone was described in chapter 2 following the procedure developed by Moynihan and Robert ${ }^{17}$.

\subsubsection{Synthesis of SNAP-PVC}

The detailed procedure for synthesizing SNAP-PVC has been described previously in chapter 2. Primary amine groups are first attached to PVC to form an aminated PVC compound which is described by Tinkilic et $\mathrm{al}^{18}$. Briefly, PVC is suspended in methanol along with ethylenediamine and triethylamine and is refluxed at $60^{\circ} \mathrm{C}$ for 2 hours then filtered. The resultant aminated PVC can then be reacted with NAP-thiolactone to allow a free thiol group to be nitrosated by t-butyl nitrite. 


\subsubsection{Synthesis of SNAP-HPAMAM}

The synthesis procedure for SNAP-HPAMAM was described in more detail previously in chapter 3 . The core hyperbranched molecule followed a modified procedure popularly used for developing polyamidoamine (PAMAM) dendrimers ${ }^{19}$.

Ethylenediamine dissolved in methanol was used as the core molecule. Methyl acrylate was then added slowly to give a 4 armed, ester terminated molecule. The solution was rotary evaporated until a viscous product was yielded, and dissolved into methanol once again. Ethylenediamine was then added to give a 4 armed amine terminated molecule. The process is then repeated until a generation 1,8 armed amine terminated molecule was formed. Each of the primary amine groups was then reacted with NAP-thiolactone and nitrosated with t-butyl nitrite to form SNAP-HPAMAM.

\subsection{Characterization}

Both materials, SNAP-PVC and SNAP-HPAMAM, were characterized using FTIR, MALDI-TOF and NMR. The nitrosated compounds were tested for total NO capacity using tri-iodide reduction. Further quantification was also done on the polymers to verify the presence of important functional groups as the synthesis steps progress. The two main functional groups were identified were primary amines and thiols. ATTOTAG FQ tested for primary amines for the HPAMAM and aminated PVC compounds, and Ellman's test for free thiols once NAP-thiolactone was covalently attached to the amine sites. The characterization results for both of the materials have been covered more in depth previously in chapters 2 and 3.

\subsubsection{Nitric Oxide Release}

NO release was recorded continuously through a Sievers $280 \mathrm{i}$ nitric oxide analyzer (NOA) by chemiluminescence. Determining the NO capacity of the materials was done by tri-iodide reduction following a protocol developed by Yang et al. ${ }^{20}$. 


\subsubsection{Polymer Film Casting and Implantation Procedure}

The solutions used to make the polymer films contained 2 weight $\%$ PVC and SNAPPVC while SNAP-HPAMAM was blended into a solution of PVC at $13 \mathrm{mg} / \mathrm{mL}$. Both SNAP based polymers were encapsulated with thin layers of PVC to prevent any unwanted diffusion of SNAP-HPAMAM into the surrounding tissue after implantation and to ensure the material contacting the tissue was consistent with the control polymer. All of the materials were dissolved in N,N-dimethylacetamide and cast into films under low humidity conditions. PVC, SNAP-PVC, and SNAP-HPAMAM PVC were then cut into $10 \mathrm{~mm}$ diameter films with an average thickness of $0.1 \mathrm{~mm}$. The materials were implanted subcutaneously away from the initial incision site into four Sprague-Dawley rats. Implantation procedures were approved by Michigan Technological University's Internal Animal Care and Use Committee (IACUC).

\subsubsection{Hematoxylin and Eosin}

Hematoxylin and eosin was used to observe the cell morphology and infiltration around the surrounding implants along with collagen formation. After formalin fixation, slides were washed with three changes PBS for 5 minutes. The slides were then rinsed with deionized water for 5 minutes. Gills-3 Haematoxlin solution was added onto each slide for approximately 2-5 minutes until overstained. Specificity of the Gills-3 staining was done by dipping the slides into an acidified solution of $\mathrm{HCl}$ and distilled water $(\mathrm{pH}=$ 1.8-2.0) five times. The slides were then rinsed in 95\% ethanol solution. Eosin Y working solution $(0.25 \%)$ was used as a counter-stain and was placed onto the slides for 45 seconds. They were then dehydrated in two changes of absolute ethanol for 5 minutes each and cleared twice with xylene substitute for 5 minutes. The slides were mounted using Eukitt mounting medium and then imaged once dried.

\subsubsection{Masson's Trichrome}


Detection of fibrous collagen content was accomplished by using Masson's trichrome stain. This stain allows for accurate measurement and recognition of the collagen fibrous capsule around the implant area. Weigert's iron hematoxylin solution was first prepared by dissolving $1 \mathrm{~g}$ of hematoxylin in $100 \mathrm{~mL}$ of $95 \%$ ethanol. A separate solution containing $29 \% \mathrm{w} / \mathrm{v} /$ ferric chloride in deionized water was made and $4 \mathrm{~mL}$ was added to $95 \mathrm{~mL}$ of deionized water and $1 \mathrm{~mL}$ of $1 \mathrm{M} \mathrm{HCl}$. The slides were thoroughly rinsed with deionized water to remove any excess formalin and Polyfreeze medium. Once rinsed, the slides were placed in the working Weigert's iron hematoxylin for 5 minutes and rinsed in running tap water for 15 minutes and then rinsed with deionized water. Bierbrich scarlet acid fuschin solution was placed on the slides for 10 minutes and differentiated using an equal parts mixture of phosphomolybdic and phosphotungstic acid until the adventitial collagen in the tissue sections no longer appeared red. The slides were then placed in aniline blue solution for 10 minutes and rinsed in distilled water for 2 minutes. Differentiation was done by placing the slides in a solution of $1 \%$ acetic acid and checked microscopically to determine staining efficiency. A quick dehydration in 95\% ethanol and absolute ethanol was done before clearing in xylene and mounted.

\subsubsection{Toluidine Blue}

Mast cells surrounding the implant area were identified by toluidine blue. Activated mast cells can be observed through the degree of degranulation and is stained purple. A stock solution was prepared by dissolving $1 \mathrm{~g}$ of toluidine blue in $70 \%$ ethanol. The working solution contained $5 \mathrm{~mL}$ of the stock solution in $45 \mathrm{~mL}$ of $1 \%$ sodium chloride solution with an adjusted $\mathrm{pH}$ of 2.4 using $1 \mathrm{M} \mathrm{HCl}$ to adjust. Slides were hydrated in three changed of deionized water for 5 minutes each and placed in the working solution for 5 minutes. They were then dipped 3 times in 95\% ethanol and 5 times in 100\% ethanol to dehydrate. Once dehydrated, the slides were dipped 20 times in a solution containing $50 \%$ acetone and $50 \%$ xylene and then followed up by dipping them 20 
times in a solution containing pure xylene before mounted in resinous mounting medium.

\subsubsection{Immunofluorescence}

A modified protocol from Abcam was used to prepare and label the tissue sections. CD163 and iNOS antibody markers were used to differentiate between M2 and M1 macrophages respectively. Total inflammatory cells were quantified by using CD11b. To enhance the signal, streptavidin-biotin antigen detection was employed after the primary antibody markers were attached to the tissue sections. DAPI was then used to label cell nuclei and verify the presence of macrophages.

For CD11b and CD163 staining, frozen sections were first allowed to warm to room temperature from $-80^{\circ} \mathrm{C}$ before being fixed in $10 \%$ formalin solution for 10 minutes. The slides were then washed in three changes of PBS for 5 minutes each and then placed in a solution of PBS containing 0.05\% TWEEN 20 for 30 minutes. After these washing steps, avidin blocking solution was placed onto the sections for 15 minutes and then washed in 2 changes of PBS for 5 minutes each. The same procedure was then repeated for the biotin blocking solution. A solution of $10 \%$ goat serum in PBS was placed onto the slides for 30 minutes once the avidin/biotin blocking was complete. No PBS washing step is done after the application of the goat serum solution. The primary antibody was then added to the slides with a dilution of 1:500 with 5\% goat serum in PBS and allowed to stay on the slides overnight at $4^{\circ} \mathrm{C}$. The slides must be placed in a close container containing water to ensure the sections do not dry overnight. The slides are then washed three times with PBS for 5 minutes each. The appropriate biotinylated IgG was placed onto the sections for 15 minutes. For the experiments described, since goat serum was used as the blocking buffer and the primary antibodies were mouse based, biotinylated goat anti-mouse IgG was used. The slides were again washed with PBS three times for 5 minutes each. Alex Fluor 633 streptavidin was diluted to a 1:400 ratio in PBS and placed onto the slides for 1 hour, protected from light during the entire time. While still being protected from light, another set of three PBS washed for 5 
minutes were done. A DAPI solution containing $5 \mathrm{mg} / \mathrm{mL}$ was further diluted to 0.5 $\mu \mathrm{L} / \mathrm{mL}$ and placed onto the slides for 1 minute. Two washed of PBS were done at 5 minutes and then were mounted with aqueous fluorescent mounting media and allowed to dry for 30 minutes before imaging.

iNOS staining used a similar procedure but did not follow the streptavidin-biotin staining method. The samples also used BSA as a serum buffer in place of goat serum and $0.01 \%$ triton X-100 was substituted for TWEEN 20, and was allowed to only rinse for 10 minutes. Before staining, antigen retrieval was done by placing the slides into a solution of citrate buffer heated at $95^{\circ} \mathrm{C}$ for 15 minutes. The primary antibody concentration was also increased to a 1:200 ratio as well.

\subsubsection{Analytical Methods}

Images of the stained sections were done on an Olympus BX51 microscope. For CD11b and CD163 labeled tissue, the images to be analyzed were taken on the 20x objective around the entire area of the implant. Counting cell nuclei and macrophages was done using CellProfiler software ${ }^{21,22}$. iNOS labeled sections were taken on the $60 \mathrm{x}$ objective around the implant and were quantified using pixel counting of the labeled cells and comparing it to DAPI labeled cells.

\subsection{Results and Discussion}

\subsubsection{Polymer Functional Group Quantification}

Both materials had their functionality quantified as each reaction step progressed from their starting synthesized materials (aminated PVC, HPAMAM) to the NAP attached molecules (NAP-PVC, NAP-HPAMAM) to their final nitrosated product (SNAP-PVC, SNAP-HPAMAM). The primary amine sites were quantified by reacting 3-(2-(furoyl) quinoline-2-carboxaldehyde (FQ) to form a fluorescent product excited at $480 \mathrm{~nm}$ with an emission maxima at $590 \mathrm{~nm}$. NAP-thiolactone is able to covalently link to primary 
amine sites through a ring opening aminolysis mechanism. The attachment to these sites was quantified through Ellman's test for free thiols. After nitrosation, the NO capacity of the two materials were tested through tri-iodide reduction. Table 4.1 shows the quantification results of the functional groups tested which was also described in previous chapters.

Table 4.1. Quantification of important functional groups and NO capacity of SNAPPVC and SNAP-HPAMAM as each reaction step progresses.It is important to recognize the potential NO load amounts depending on amount of primary amine sites in a material. Although attaching NAP-thiolactone to every primary amine functional group is difficult, seeing the efficiency gives insight to the material kinetics. The low attachment to HPAMAM is most likely due to a large amount of steric hindrance as the material is a large network of multiple sized chains while the amines on the backbone of the PVC chains are most likely spread out and in fewer number.

\begin{tabular}{|c|c|c|c|}
\hline & $\begin{array}{c}\text { Primary Amines } \\
(\boldsymbol{\mu m o l} / \mathbf{m g})\end{array}$ & Thiols $(\boldsymbol{\mu m o l} / \mathbf{m g})$ & $\begin{array}{c}\text { Nitric Oxide } \\
\text { Capacity } \\
(\boldsymbol{\mu m o l} / \mathbf{m g})\end{array}$ \\
\hline SNAP-PVC & $0.201 \pm 0.013$ & $0.153 \pm 0.009$ & $0.0392 \pm 0.004$ \\
\hline SNAP-HPAMAM & $4.48 \pm 0.22$ & $2.74 \pm 0.30$ & $1.90 \pm 0.12$ \\
\hline
\end{tabular}

\subsubsection{NO Release Profile}

$10 \mathrm{~mm}$ diameter films were tested for passive NO release by placing them inside stirring PBS solutions at $37^{\circ} \mathrm{C}$. The initial NO release was recorded over a set amount of time to give an insight into the distinct release profile of the two NO releasing materials when in contact with PBS. The polymer films were then placed inside an incubator at $37^{\circ} \mathrm{C}$ in PBS when not recording the NO release. Films were then taken out and tested again periodically to observe if the polymers were still passively releasing NO. The schematic for how the polymer layered system functions is shown in Figure 4.1. 


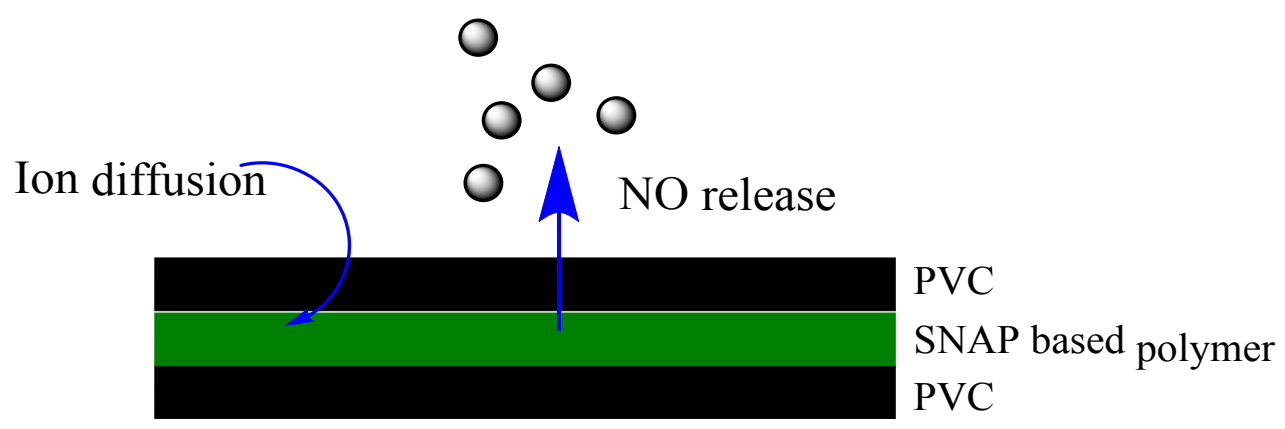

Figure 4.1. Layered polymer schematic for passive NO release when implanted subcutaneously. Since it is difficult to use the light triggering mechanisms normally seen with SNAP based materials, using ion and heat mediated release are the most convenient mechanisms for passive in vivo release. Due to PVC's affinity for ion permeability, the SNAP functional groups are able to release a substantial amount of NO when placed in physiological conditions.

Figures 4.2 and 4.3 show the NO release profiles of the two materials under physiological conditions. Comparing the two polymers' NO release profiles show SNAP-PVC exhausting most of its passive NO release over a short period of time while SNAP-HPAMAM PVC sustains a higher NO release much longer. This is due to the higher capacity that is seen in the SNAP-HPAMAM material. Its encapsulation within the PVC matrix to prevent leeching also plays a large role in its persistent NO release. Having these two types of NO release profiles to examine in vivo is important as it can give insight into the future developments of long lasting subcutaneous NO releasing materials. If the shorter term, lower NO release from SNAP-PVC was to perform well, it would demonstrate the importance of having the presence of trace amounts of NO during the acute inflammatory stages in response to a hydrophobic implant. The SNAPHPAMAM PVC also releases a majority of its NO during the acute inflammation stage, but would have the potential NO storage to affect the transition into the chronic inflammation stage. Care was taken to make sure too much NO was not being released 
as large amounts of NO can trigger apoptotic events with the cells in the surrounding implanted tissue.

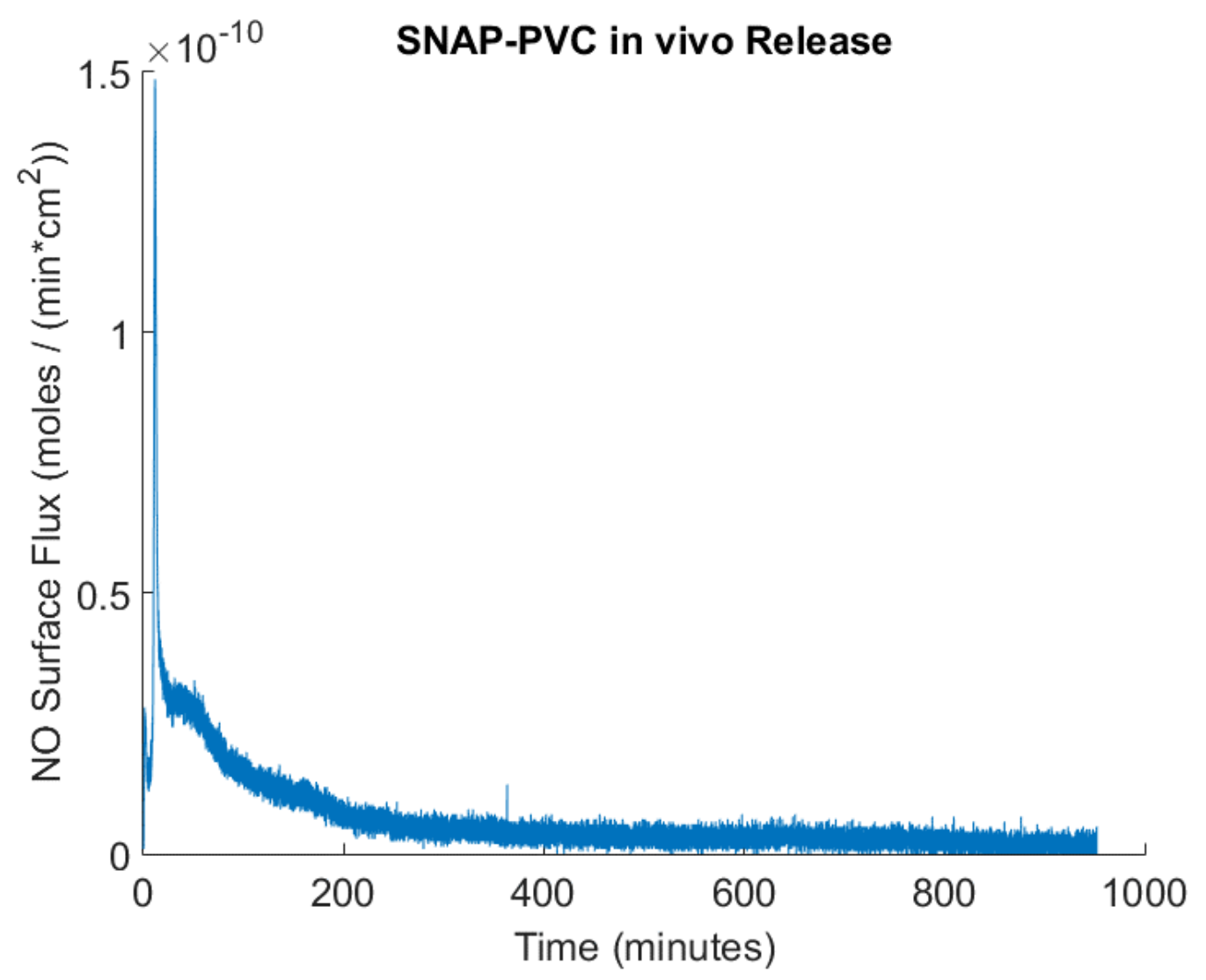

Figure 4.2. NO release profile of SNAP-PVC in PBS at $37^{\circ} \mathrm{C}$. The release profile of SNAP-PVC was very minimal compared to other NO generating materials that have been implanted. Compared to the material's release when using external triggers like light or metal ions, the passive release does not trigger NO release at a fairly high level for a long time. 


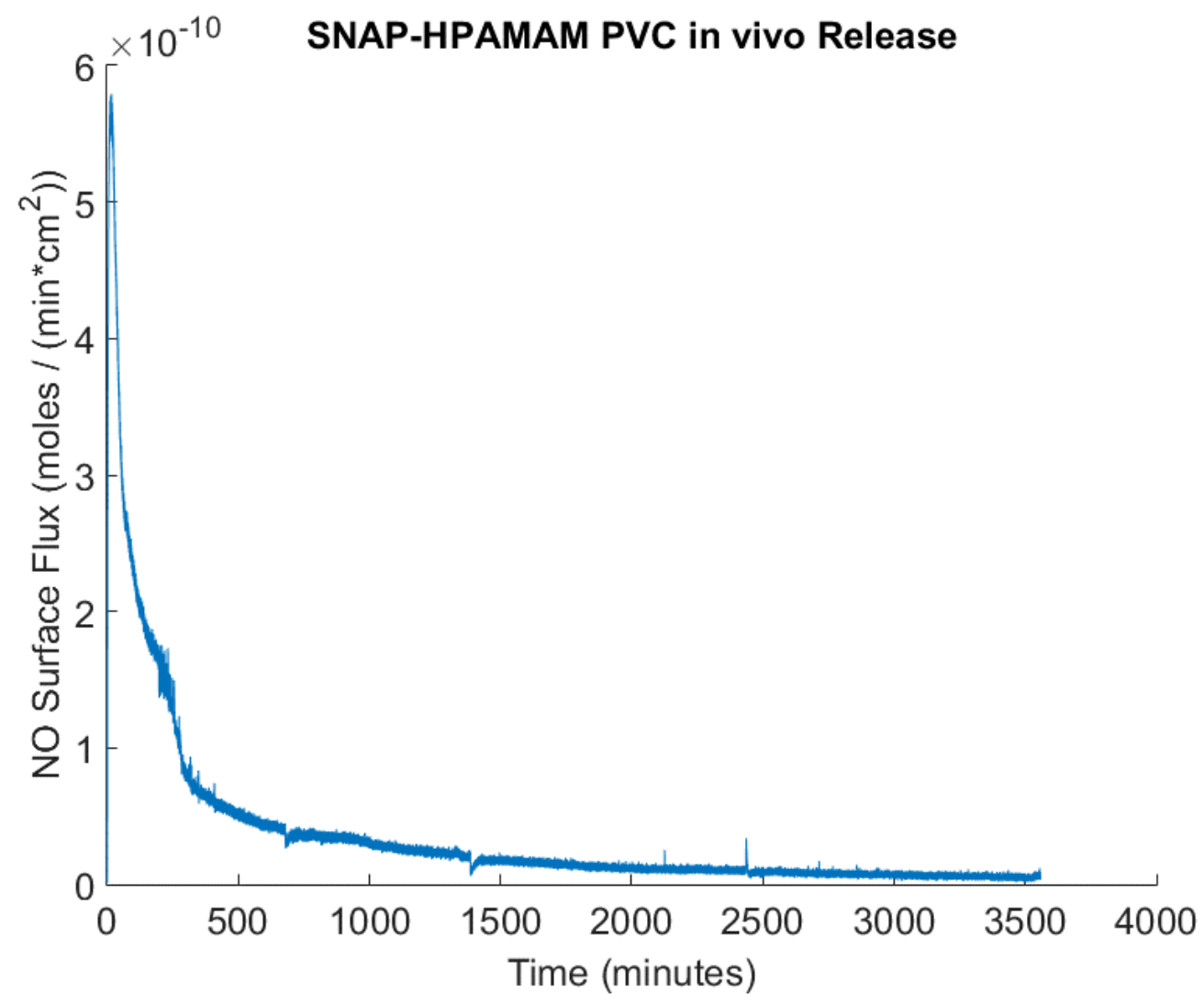

Figure 4.3. NO release profile of SNAP-HPAMAM PVC in PBS at $37^{\circ} \mathrm{C}$.Compared to the NO release from SNAP-PVC, SNAP-HPAMAM PVC demonstrated a much larger NO release over a longer period of time. This allows the comparison of two distinct NO release profiles in vivo.

\subsubsection{Masson's Trichrome Analysis}

Fibrous encapsulation thickness was recorded for the 15 day implanted NO releasing and control PVC samples and is shown in Figure 4.4. Encapsulation was quantified across 8 tissue sections from 4 different rats for each material. Only the dense, organized collagen at the implant-tissue interface was considered for measuring the thickness of the encapsulation while the loose, disorganized collagen seen away from the interface was disregarded. NO's effect on fibrous encapsulation showed a large reduction in thickness for the 15 day implants for both SNAP-PVC and SNAP- 
HPAMAM PVC when compared to controls. The collagen was also much more distinct in the control samples as seen in panels A and D of Figure 4.4. The individual collagen fibrils are much more developed and easier to identify than the collagen around the NO releasing implants.

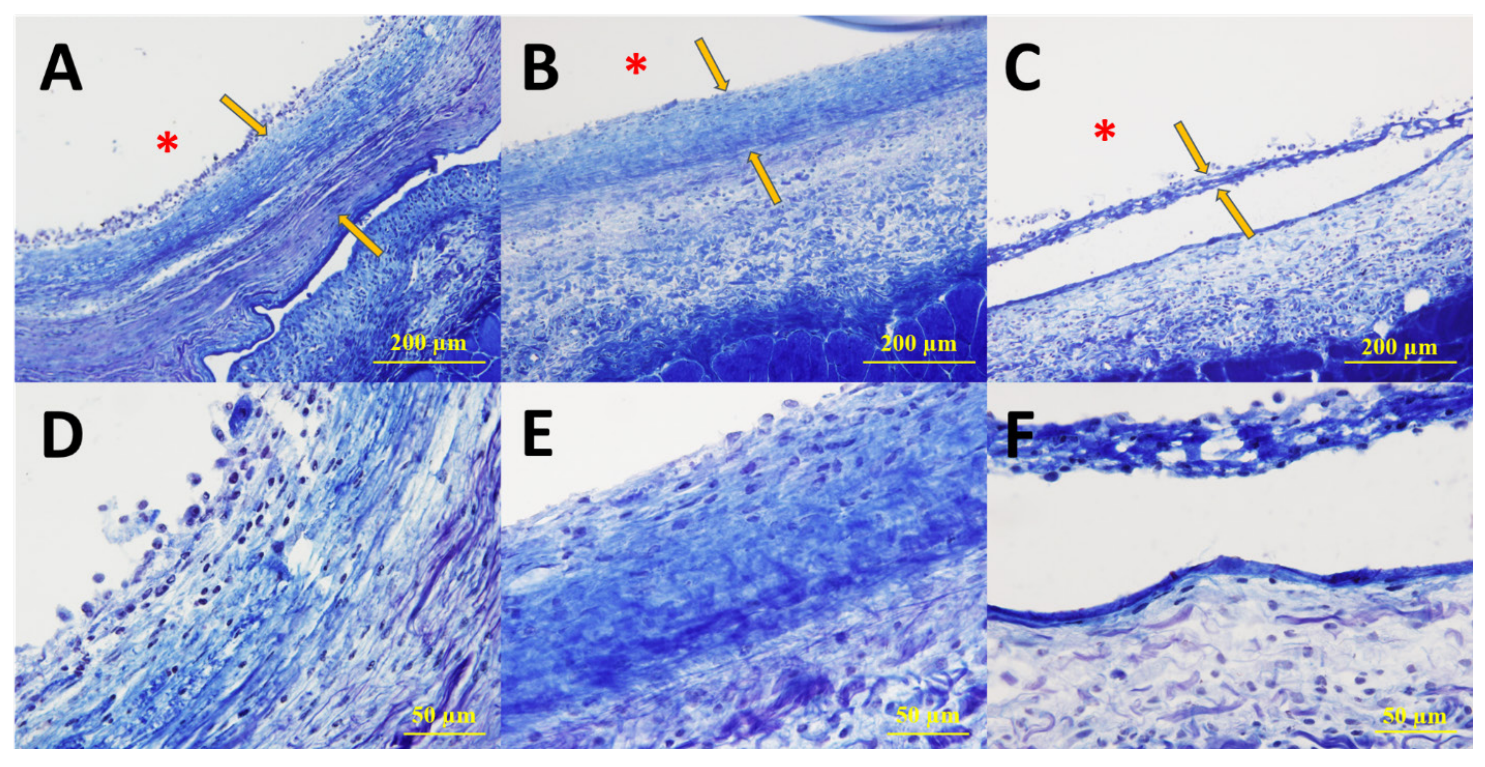

Figure 4.4. Trichrome analysis of 15 day subcutaneously implanted materials. Panels A, B, and C represent 200x images of PVC, SNAP-PVC, and SNAP-HPAMAM PVC implants respectively. Panels D, E, and F represent 600x images of PVC, SNAP-PVC, and SNAP-HPAMAM PVC implants respectively. The fibrous encapsulation thickness (yellow arrows) were measured from the implant location (red star) to the area of disorganized extracellular matrix. 


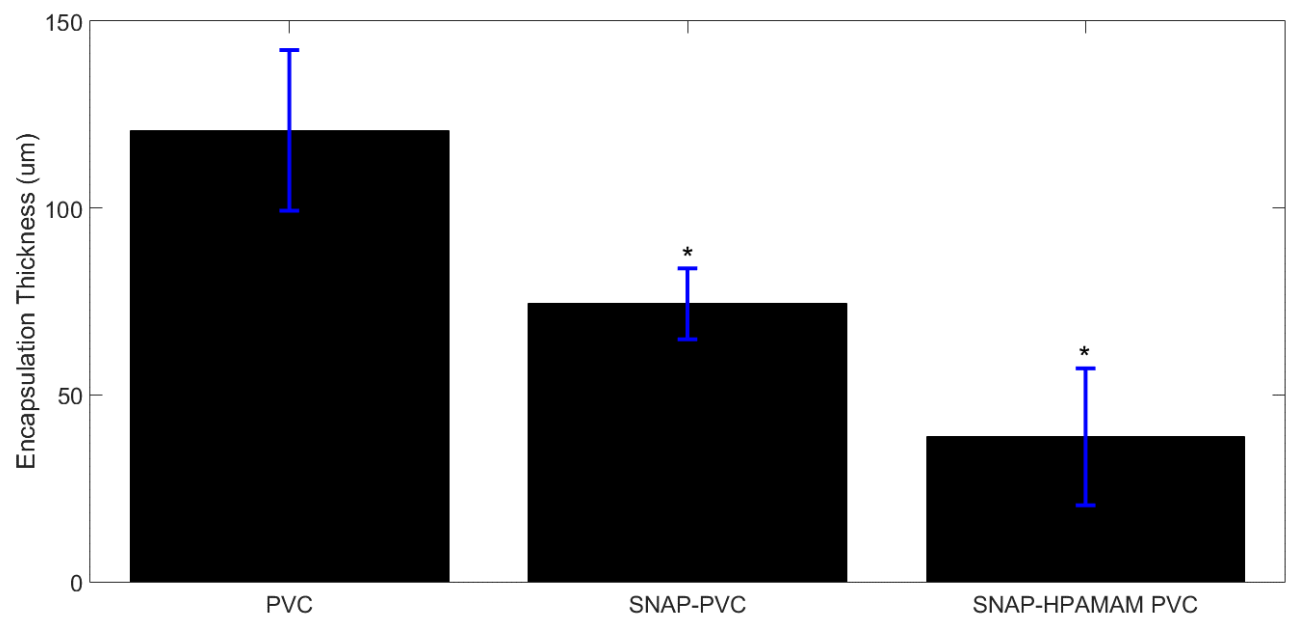

Figure 4.5. Fibrous encapsulation thickness data for PVC, SNAP-PVC, and SNAPHPAMAM PVC for 15 day implants. A dramatic reduction in the fibrosis response was seen in the NO releasing implants compared to the control PVC samples. Using $\alpha=0.05$, a significant difference in encapsulation thickness was seen for both SNAPPVC and SNAP-HPAMAM PVC when compared to PVC $(\mathrm{p}=0.0128$ and $\mathrm{p}=0.0004$ respectively).

\subsubsection{Toluidine Blue Analysis}

Mast cells play an important role in the inflammatory response as they release histamine, serotonin, along with other cytokines that recruit macrophage recruitment to a wound site ${ }^{23}$. The toluidine blue stained images are able to show degranulated mast cells versus quiescent mast cells. NO has been shown to help modulate the rate of degranulation in mast cells which can further prevent unwanted inflammatory cells from approaching an implant area ${ }^{24}$. This was demonstrated in the toluidine blue stains which showed a greater quantity of degranulated mast cells in control PVC when compared to the SNAP-PVC and SNAP-HPAMAM PVC 1 day implants. For the 15 day implants, there was not a large difference between the numbers of mast cells across all of the samples. This could be due to the NO reservoir being exhausted from both of the NO donating polymers. 


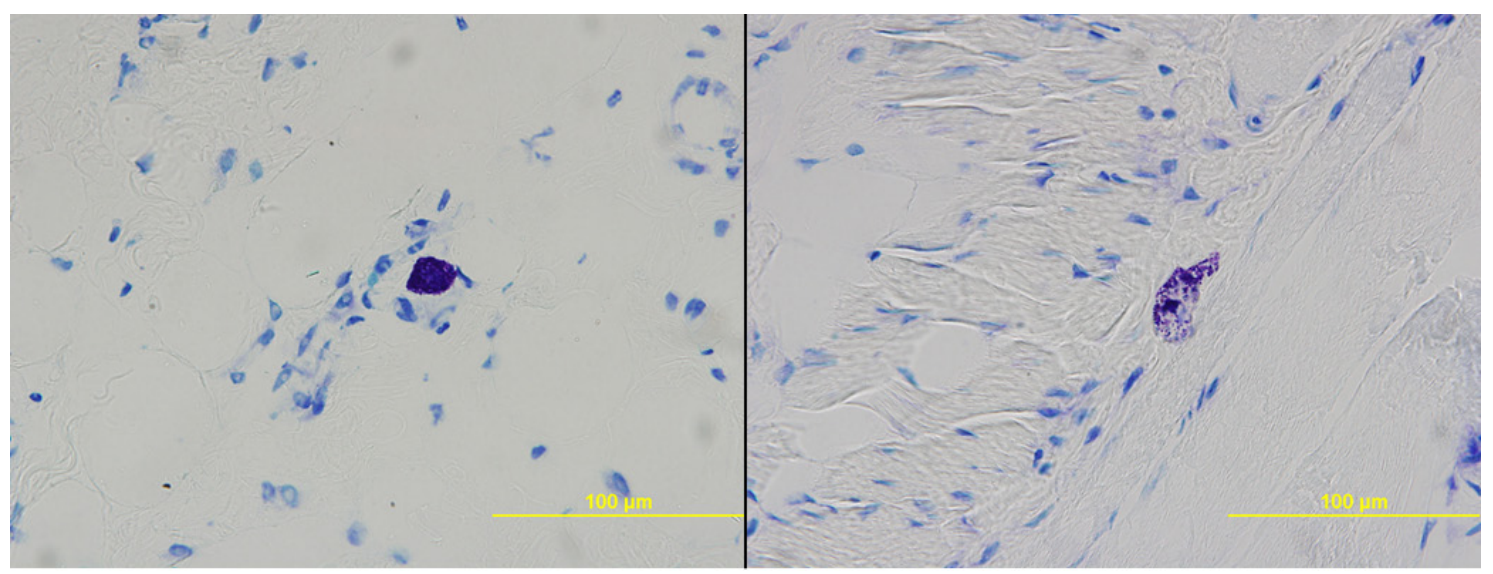

Figure 4.6. Difference between active and inactive mast cells Mast cells are labeled purple and are determined inactive (left) when they are granulated and active (right) as the degranulation begins to occur. Although all samples showed signs of degranulated mast cells, the NO releasing implants had the most inactive mast cell population, which was especially seen in the 1 day implants.

\subsubsection{Hematoxylin and Eosin Analysis}

Observing the H\&E images shows a large range of different cell types present in the one day implants. As seen with acute inflammation, a large population of neutrophils and monocytes have migrated to the implant site along with the presence of eosinophils in the 1 day implants (not shown). The control PVC implants showed higher infiltration of cells to the implant area when compared to the NO releasing implants. This result correlates to the quantity of mast cell degranulation between the materials. It becomes much easier for inflammatory cells to migrate to a wound site the greater the mast cell activity.

The beginning stages of fibrosis can also be seen in the 15 day implants as chronic inflammation begins to persist. This is seen especially in the control polymer implants. Compared to the control, the NO releasing polymers show a much more resolved tissue area around the interface. Control PVC also displays an increase of inflammatory cells at the implant-tissue interface and the beginning stages of fibrosis is expressed more 
prominently. The SNAP-HPAMAM PVC implant interface has little to no inflammatory cells on the implant-tissue interface to the same degree as control PVC implants.

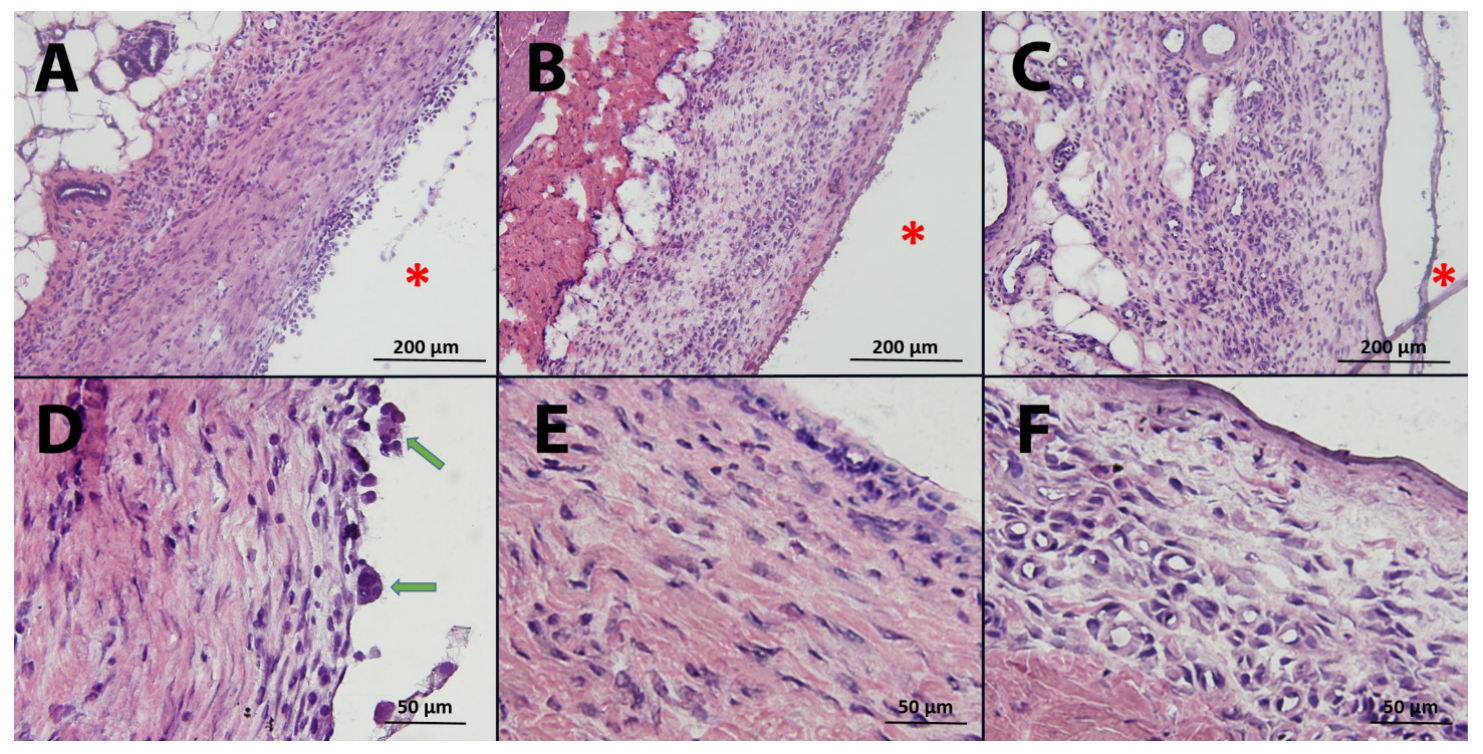

Figure 4.7. H\&E analysis of 15 day subcutaneously implanted materials. Panels A, B, and $\mathrm{C}$ represent the 200x images of PVC, SNAP-PVC, and SNAP-HPAMAM PVC respectively. The 600x images are then shown in panels D, E, and F for PVC, SNAPPVC, and SNAP-HPAMAM PVC respectively. The green arrows in panel D show the presence of foreign body giant cells at the tissue-implant interface. Red stars indicate the implant locations.

\subsubsection{Macrophage Immunofluorescence}

Quantification of the number and phenotype of macrophages was done around the implantation site. Total number of granulocytes like neutrophils, monocytes, and macrophages were identified using CD11b while M1 macrophages were specifically identified using iNOS antibodies. CD163 was used to quantify and locate M2 macrophage activity around the implants. All quantification data was done for the 15 day implants only. High expression of CD11b and iNOS in cells has been proven to be an indication of pro-inflammatory M1 macrophages ${ }^{25}$. CD11b expression also directly 
relates to the release of pro-inflammatory cytokines, where high expression has demonstrated a release of TNF- $\alpha$ and IL- $1 \beta^{26}$. For any implantable device, the presence of M2 macrophages is highly preferred as they mediate and control inflammation along with tissue remodeling while the presence of the M1 phenotype encourage chronic inflammation. M2 macrophages also help remodel tissues by producing certain MMPs and produce VEGF to promote blood vessel fusion and formation ${ }^{27}$.

A noticeable difference between the two macrophage phenotypes and total number of granulocytes was seen between the NO releasing implants and control implants. Along with the quantity present around the implant interface, the location of the specific macrophage phenotypes was also observed. For the 15 day PVC control implants, $\mathrm{iNOS}^{+}$macrophages have a high density around the majority of the polymer-tissue interface area while $\mathrm{CD}_{163^{+}}$macrophages seem to be much less frequent overall. The 15 day NO releasing PVC implanted materials showed a much lower concentration of iNOS $^{+}$macrophages near the implant. The aggregation of macrophages at the interface was less consistent than the aggregation around the control PVC sections. The presence of $\mathrm{CD} 163^{+}$macrophages in the $\mathrm{NO}$ releasing PVC was also more prevalent overall when compared to the control PVC. When observing the iNOS stained sections for the NO releasing polymer implants, there are cells at the implant-tissue interface that stain negatively for iNOS while control sections have a uniform labeling of $\mathrm{iNOS}^{+}$cells around the majority of the interface. This signifies a possible switch in phenotype of the migrating M1 macrophages due to the release of NO. There is also the possibility that they are still M1 macrophages but are not as active.

There is also more polymer fragmentation present in the control PVC implants than the NO releasing implants. iNOS and CD11b images show a high density of macrophages surrounding these polymer fragments within the tissues indicating frustrated phagocytosis. The SNAP-PVC showed a minor amount of polymer fragmentation compared to the control PVC, while the SNAP-HPAMAM PVC showed none at all. NO in high enough concentration has the ability to decrease the amount of chronic inflammatory cytokine release from macrophages which then lowers the recruitment of 
macrophages to the material interface ${ }^{28}$. In the absence of $\mathrm{NO}$, these cytokines are upregulated to allow the migration of more macrophages to the implantation site along with macrophage fusion. Eventually this fusion of macrophages leads to the formation of foreign body giant cells (FBGCs), which will release harmful degradative enzymes to attempt to break apart the polymer ${ }^{29-30}$. As seen in the H\&E stain in Figure 4.7, FBGCs and the total number of cells at the interface are much higher in control PVC.

\subsubsection{Macrophage Phenotype Quantification}

Observations were made on the images taken with respect to macrophage number and phenotype based on NO release. Images were taken at 200x magnification around the implant area for both $\mathrm{CD} 11 \mathrm{~b}^{+}$and $\mathrm{CD} 163^{+}$sections and were counted using CellProfiler software. An independent samples student t-test was then done to compare the presence of $\mathrm{CD} 11 \mathrm{~b}^{+}$and $\mathrm{CD} 163^{+}$cells in control PVC implants to SNAP-PVC and SNAPHPAMAM PVC implants. Quantifying the iNOS ${ }^{+}$cells proved to be more difficult, as iNOS stains for cytoplasmic features within macrophages while CD11b and CD163 both stain for membrane bound proteins, which are much easier for cell counting programs to identify. Pixel counting was used instead to quantify the iNOS activity within the tissue sections using ImageJ and Adobe Photoshop. The positive iNOS pixels in the green channel were compared to the DAPI pixel count in the blue channel and the ratio between the two was used to compare the overall iNOS activity around the implant sites under 600x magnification.

For the 15 day implants, a no significant difference was seen in $\mathrm{CD}_{11} \mathrm{~b}^{+}$or $\mathrm{CD} 163^{+}$cells when comparing control PVC implants when to both the SNAP-PVC and SNAPHPAMAM PVC implants. Although there was no significant difference, there is an observable trend in a decrease of $\mathrm{CD} 11 \mathrm{~b}^{+}$cells with the increased in NO delivery from the SNAP-PVC and SNAP-HPAMAM PVC along with a slightly increased trend in $\mathrm{CD} 163^{+}$cells for the SNAP-HPAMAM PVC. This increase in M2 macrophage population coincides with the fact that the implant area contained the lowest population of granulocytes and M1 macrophages, demonstrating their ability to control and 
decrease the inflammatory response to a wound site or implant. By referring back to Figure 4.7, panel F, the consequences of a higher population of $\mathrm{CD} 163^{+}$cells can be seen from the H\&E stain. There is much less matrix formation along with higher vasculogenesis around the implant area, which could be caused from the secretion of VEGF from active M2 macrophages. This trend also been proven previously using CD31 staining to demonstrate the angiogenesis that occurs around NO releasing subcutaneous implanted materials ${ }^{13}$.

The one day implants showed nearly identical amounts of cells marked with both

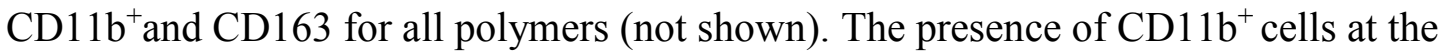
initial stages of implantation are expected to be the same as NO's main effect is to hinder pro-inflammatory cytokines that further the propagation and recruitment of additional macrophages. The $\mathrm{CD} 11 \mathrm{~b}^{+}$cells are most likely neutrophils, monocytes, and eosinophils along with the initial differentiating macrophages for the 1 day implants. This could also factor in to the lack of statistical difference between the materials as the stages of acute inflammation is relatively consistent and necessary part of the wound healing process.

For quantification of $\mathrm{iNOS}^{+}$cells around the implant, 600x images were taken around the tissue-implant interface and a pixel count ratio between iNOS stained features (green) and DAPI labeled cells (blue) was done. Since iNOS is labeled within the cytoplasm of macrophages, individual counting would prove to be difficult. Active macrophages will display a strong iNOS signal that can be accurately quantified. To observe the difference in signal, RAW 264.7 passage 13 murine macrophages were treated with LPS and stained with iNOS and is shown in Figure 4.8. The LPS treated macrophages simulate their morphology when active. When comparing the macrophages at the interface in PVC control implants to the NO releasing implants, there is a distinct difference in the macrophage morphology and is shown in Figure 4.9. The PVC control shows a very similar active phenotype similar to the LPS treated macrophages. This extreme activity is also seen around polymer fragmentation that occurs which is shown in Figure 4.10. The large standard deviations seen in the PVC 
quantification is due to these fragmentation events that occurred in two of the PVC implants, which then greatly increased the presence of $\mathrm{iNOS}^{+}$cells around the implant area. SNAP-PVC still demonstrated a region of active M1 macrophages at the interface, but the overall activity of them was much less than the PVC interface. When comparing the iNOS/DAPI ratio between PVC and SNAP-PVC, there was no significant difference seen. Even with less active macrophages at the interface, there were still a large number around the tissue area that stained positive for iNOS. This demonstrates that the passive NO release from SNAP-PVC was not enough to hold off the overall aggressive macrophage inflammation to the implant. SNAP-HPAMAM PVC implants demonstrated a less active interface area along with a low macrophage count in the surrounding tissue. In this case, a significant reduction of the iNOS/DAPI ratio was seen when comparing SNAP-HPAMAM to PVC.

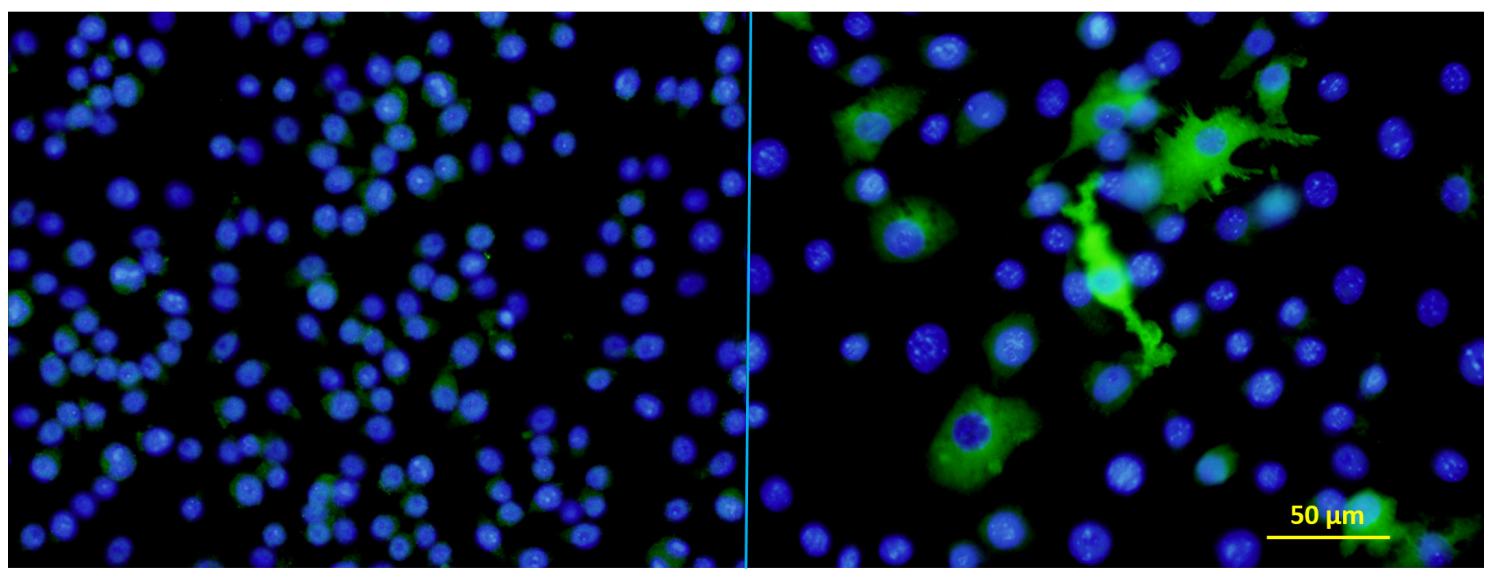

Figure 4.8. Cell culture of RAW 264.7 murine macrophages stained with iNOS at 600x.LPS treated (right image) and non-LPS treated (left image) demonstrate the difference in iNOS staining activity. More active macrophages have a much larger cytoplasm filled with iNOS enzymes. 

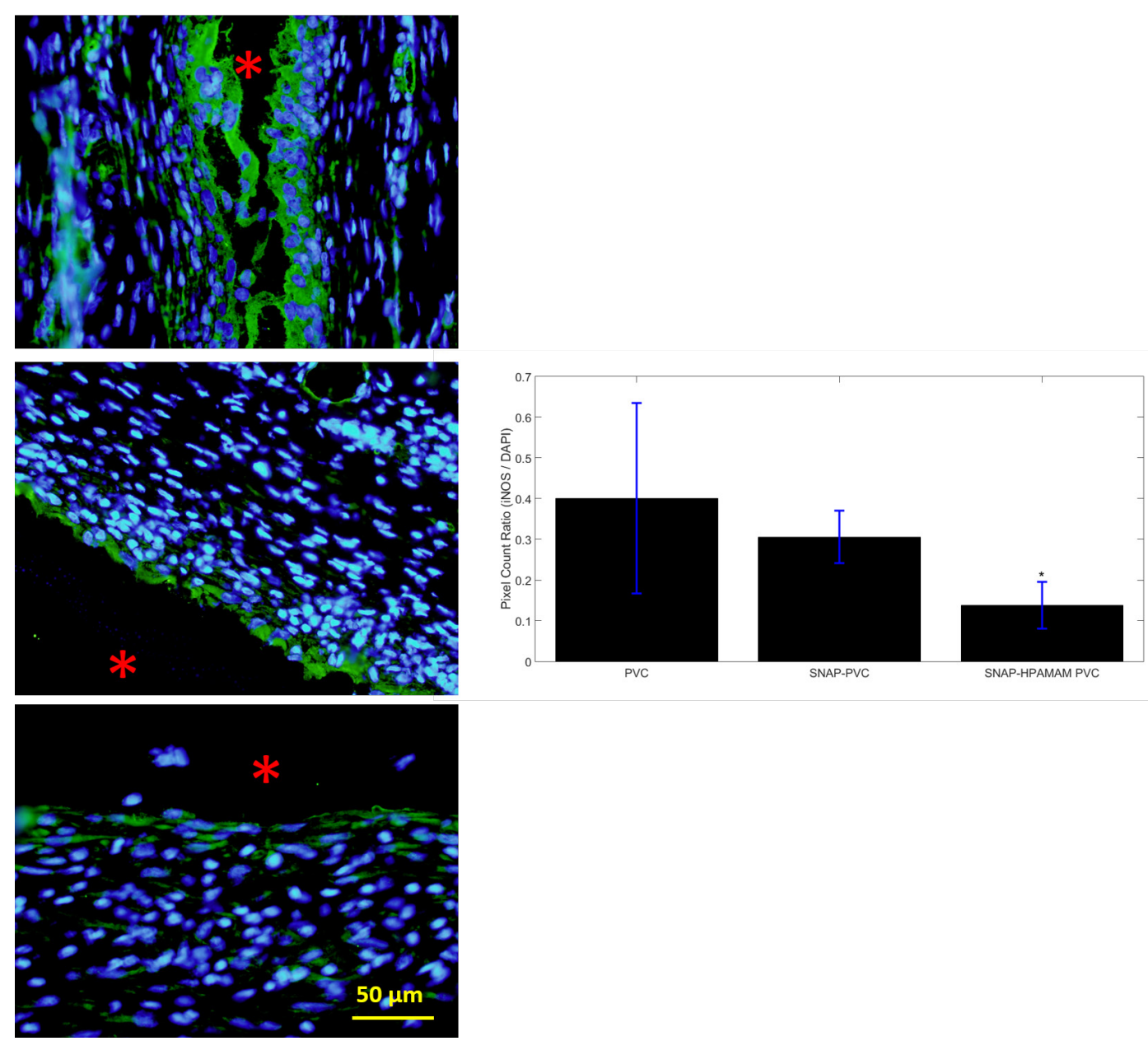

Figure 4.9. Immunofluorescence of iNOS $^{+}$cells (green) around 15 day implants.PVC control (top) displayed very active macrophages, especially at the implant interface. SNAP-PVC (middle) also showed a lot of activity, but with smaller iNOS ${ }^{+}$cytoplasmic features. SNAP-HPAMAM PVC (bottom) had the lowest amount of iNOS activity from macrophages, and only showed aggregation of macrophages at the interface in rare occaisions. SNAP-HPAMAM stained macrophages were mostly seen away from the interface area. A signfiicant difference in the ratio of iNOS/DAPI was seen when comparing PVC to SNAP-HPAMAM PVC $(p=0.0312)$, but no significant difference was seen between SNAP-PVC and PVC $(p=0.2897)$. Red stars indicate the implantation area. Red stars denote the implant location. 


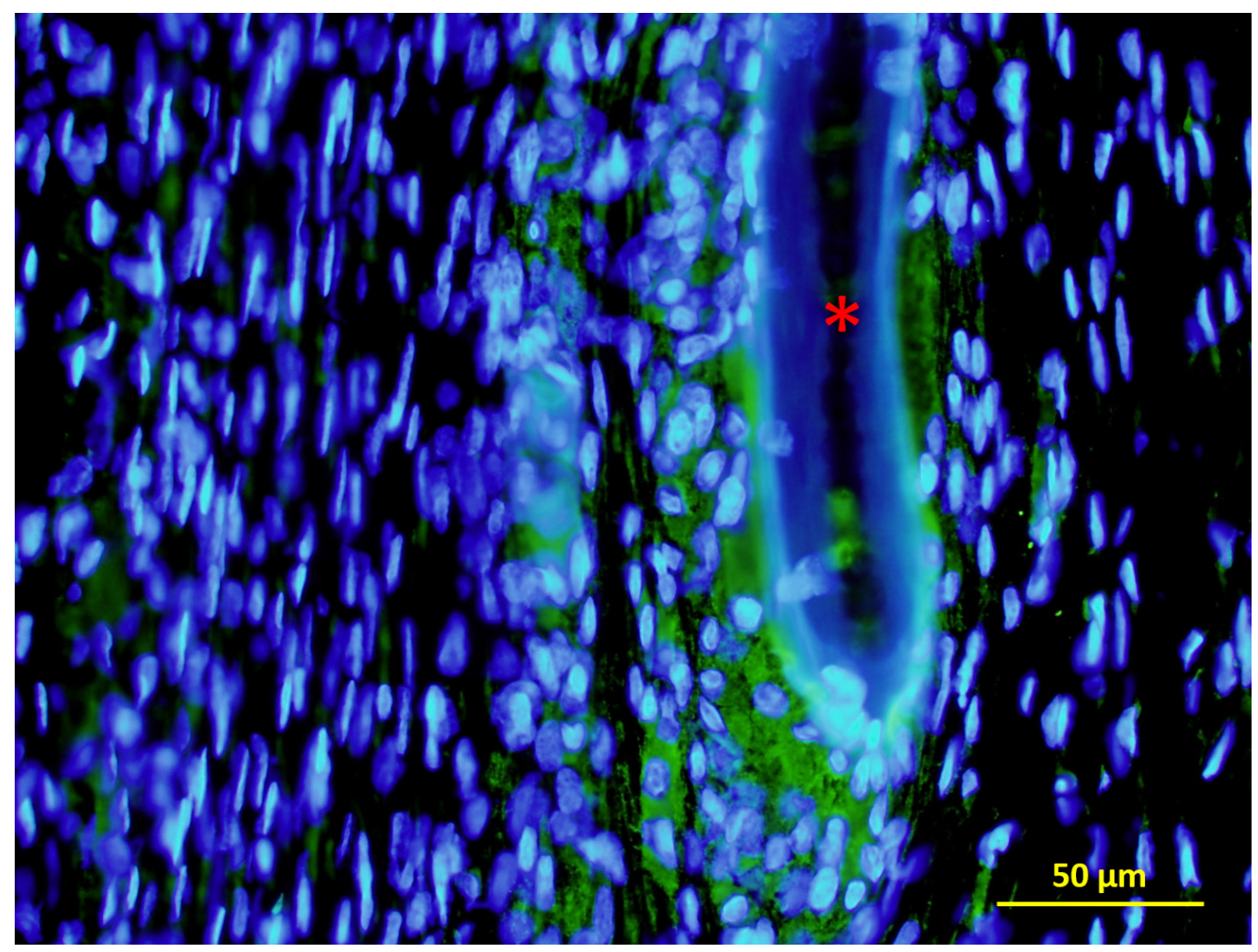

Figure 4.10. Inflammatory response to polymer fragments seen in 15 day PVC implants. The aggregation of iNOS labeled cells (green) show the consequences of the increased, harsh inflammatory response to the control PVC implants as these tissue sections showed the most polymer fragmentation (red star). Image was taken at $600 \mathrm{x}$ magnification. 


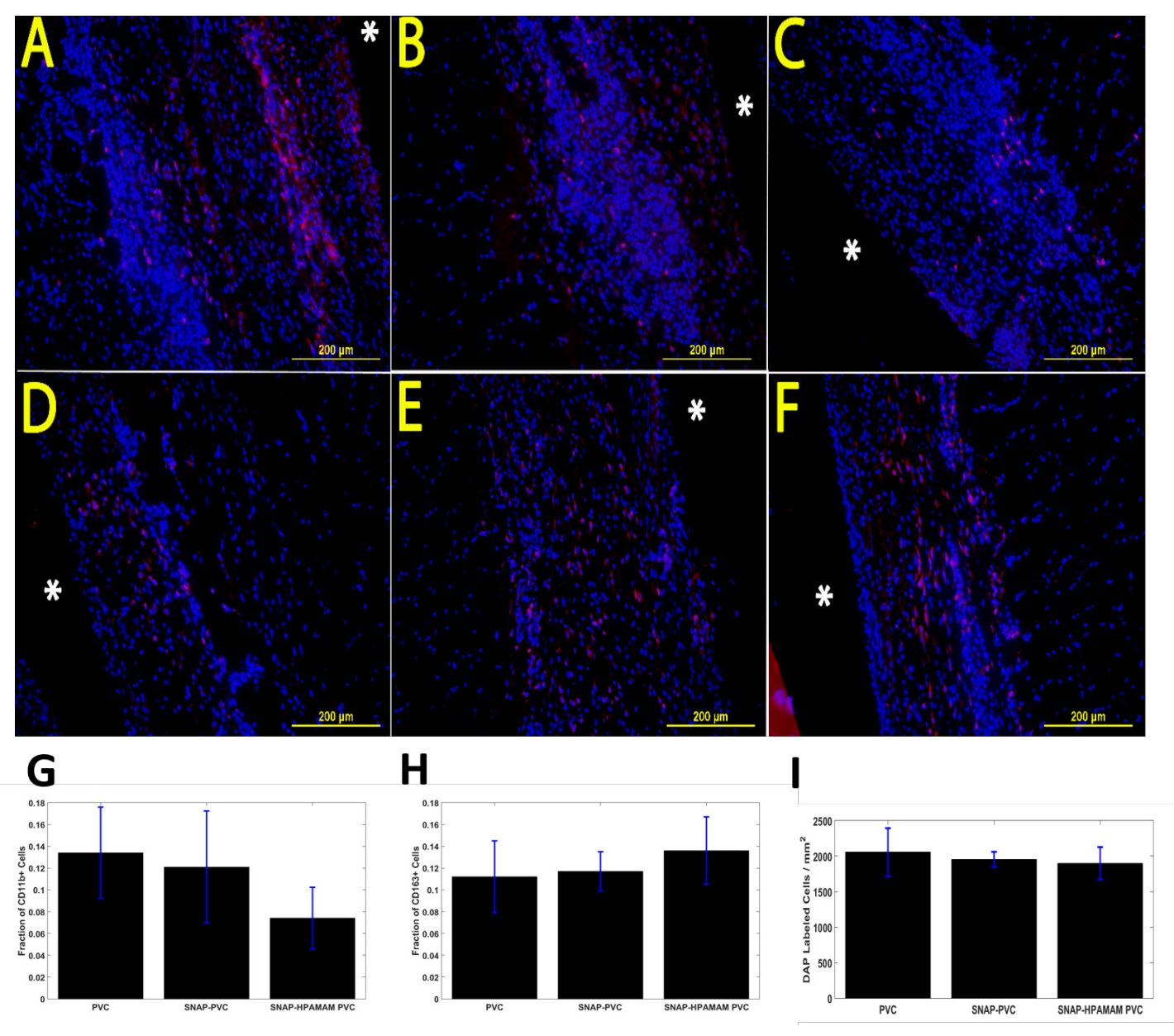

Figure 4.11. Immunofluorescence and analysis of cell nuclei, CD11b, and CD163 marked cells for 15 day implants.Aggressive and inflammation inducing cells were marked with CD11b (red) for PVC (A), SNAP-PVC (B), and SNAP-HPAMAM PVC

(C) while cell nuclei were stained with DAPI (blue). Anti-inflammatory, M2 macrophages were marked with CD163 (red) for PVC (D), SNAP-PVC (E), and SNAPHPAMAM PVC (F). White stars indicate the locations of the implant. Quantification of the tissue around the implants shows no significant reduction in $\mathrm{CD} 11 \mathrm{~b}^{+}$or $\mathrm{CD} 163^{+}$ cells for both NO releasing implants, but a trend was observed in the quantification for $\mathrm{CD} 11 \mathrm{~b}(\mathrm{G})$ to demonstrate a reduction in the overall cell count. $\mathrm{CD} 163^{+}$macrophages also saw a slight increase in quantity with increased exogenous NO delivery $(\mathrm{H})$ when

compared to control PVC. Total cell count was also reduced around the SNAPHPAMAM implant compared to PVC (I). An alpha level of 0.05 was used for all statistical testing. Error bars represent one standard deviation.

The results from these experiments demonstrate slightly different results from in vitro experiments that were done in the past. Human mononuclear cells have been shown to express pro-inflammatory cytokines such as TNF and IL-1 when incubated with 100 
uM levels of SNAP NO donors, suggesting a phenotypical switch to an M1 state if concentrations are high enough ${ }^{31}$. However, these levels of $\mathrm{NO}$ were not released from the implanted materials over the time periods that are being described. The in vitro studies of utilizing $\mathrm{NO}$ donors in this manner implies all of the NO is being administered at once. Using an NO donating material that is able to keep a lower, more steady flux of NO over a period of time would elicit a different response. This suggests a different possible mechanism for migrating blood monocytes towards a region of more controlled, lower NO release rather than an entire saturated environment of SNAP NO donors.

\subsection{Conclusion}

Two different NO releasing PVC based polymers that released different total amounts of $\mathrm{NO}$ were able to elicit different immune responses compared to control PVC based on the level and duration of $\mathrm{NO}$ being emitted into the surrounding tissue. While SNAP-PVC and SNAP-HPAMAM PVC implants were not able to significantly reduce the amount $\mathrm{CD} 11 \mathrm{~b}^{+}$and $\mathrm{CD} 163^{+}$cells, there was a general trend occurring for these cell types with the two specific NO release profiles. More rats would need to be tested in future studies to further verify these trends. There was a significant reduction in the fibrous encapsulation area around the implants for both NO releasing polymers, proving that even small amounts of exogenous NO release is capable of reducing fibrosis to implants. The passive NO release from SNAP-PVC was too low for the implantation duration seen to give impressive in vivo results when observing the overall presence of iNOS around the implants. Another external factor would have to be used to trigger the NO release from the SNAP-PVC polymer to elicit a more favorable anti-inflammatory response. However, SNAP-HPAMAM PVC was able to see a significant reduction in $\mathrm{iNOS}^{+}$cell activity, demonstrating that the proper NO flux is able to lower this type of aggressive macrophage interaction. Future studies could be done using an even higher NO capacity polymer film utilizing SNAP-HPAMAM blends over longer periods of time. 


\subsection{References}

1. Anderson, J. M.; Rodriguez, A.; Chang, D. T. In Foreign body reaction to biomaterials, Seminars in immunology, Elsevier: 2008; pp 86-100.

2. Coleman, J. Nitric oxide: a regulator of mast cell activation and mast cell-mediated inflammation. Clinical \& Experimental Immunology 2002, 129 (1), 4-10

3. McNally, A. K.; Anderson, J. M. Interleukin-4 induces foreign body giant cells from human monocytes/macrophages. Differential lymphokine regulation of macrophage fusion leads to morphological variants of multinucleated giant cells. The American journal of pathology 1995, 147 (5), 1487.

4. Haas, A. The phagosome: compartment with a license to kill. Traffic 2007, 8 (4), 311-330.

5. Anderson, J. M. Biological responses to materials. Annual Review of Materials Research 2001, 31 (1), 81-110.

6. Palmer, R. M.; Ferrige, A.; Moncada, S. Nitric oxide release accounts for the biological activity of endothelium-derived relaxing factor. 1987.

7. Mowery, K. A.; Schoenfisch, M. H.; Saavedra, J. E.; Keefer, L. K.; Meyerhoff, M. E. Preparation and characterization of hydrophobic polymeric films that are thromboresistant via nitric oxide release. Biomaterials 2000, 21 (1), 9-21.

8. Frost, M. C.; Rudich, S. M.; Zhang, H.; Maraschio, M. A.; Meyerhoff, M. E. In vivo biocompatibility and analytical performance of intravascular amperometric oxygen sensors prepared with improved nitric oxide-releasing silicone rubber coating. Analytical chemistry 2002, 74 (23), 5942-5947.

9. Nablo, B. J.; Rothrock, A. R.; Schoenfisch, M. H. Nitric oxide-releasing solgels as antibacterial coatings for orthopedic implants. Biomaterials 2005, 26 (8), 917-924.

10. MacMicking, J.; Xie, Q.-W.; Nathan, C. Nitric oxide and macrophage function. Annual review of immunology 1997, 15 (1), 323-350. 
11. Ialenti, A.; Ianaro, A.; Moncada, S.; Di Rosa, M. Modulation of acute inflammation by endogenous nitric oxide. European journal of pharmacology 1992, 211 (2), 177-182.

12. Keefer, L. K.; Nims, R. W.; Davies, K. M.; Wink, D. A. "NONOates"(1substituted diazen-1-ium-1, 2-diolates) as nitric oxide donors: convenient nitric oxide dosage forms. Methods in enzymology 1996, 268, 281-293.

13. Hetrick, E. M.; Prichard, H. L.; Klitzman, B.; Schoenfisch, M. H. Reduced foreign body response at nitric oxide-releasing subcutaneous implants. Biomaterials 2007, 28 (31), 4571-4580.

14. Heller, A. Implanted electrochemical glucose sensors for the management of diabetes. Annual Review of Biomedical Engineering 1999, 1 (1), 153-175.

15. Badylak, S. F.; Valentin, J. E.; Ravindra, A. K.; McCabe, G. P.; Stewart-Akers, A. M. Macrophage phenotype as a determinant of biologic scaffold remodeling. Tissue Engineering Part A 2008, 14 (11), 1835-1842.

16. Galván-Peña, S.; O’Neill, L. A. Metabolic reprograming in macrophage polarization. Frontiers in immunology 2014, 5.

17. Moynihan, H. A.; Roberts, S. M. Preparation of some novel S-nitroso compounds as potential slow-release agents of nitric oxide in vivo. J. Chem. Soc., Perkin Trans. 1 1994, (7), 797-805.

18. Tinkilic, N.; Cubuk, O.; Isildak, I. Glucose and urea biosensors based on all solid-state PVC-NH 2 membrane electrodes. Analytica chimica acta 2002, 452 (1), 29-34.

19. Klaykruayat, B.; Siralertmukul, K.; Srikulkit, K. Chemical modification of chitosan with cationic hyperbranched dendritic polyamidoamine and its antimicrobial activity on cotton fabric. Carbohydrate Polymers 2010, 80 (1), 197-207.

20. Yang, B. K.; Vivas, E. X.; Reiter, C. D.; Gladwin, M. T. Methodologies for the sensitive and specific measurement of S-nitrosothiols, iron-nitrosyls, and nitrite in biological samples. Free radical research 2003, 37 (1), 1-10. 
21. Carpenter, A. E.; Jones, T. R.; Lamprecht, M. R.; Clarke, C.; Kang, I. H.; Friman, O.; Guertin, D. A.; Chang, J. H.; Lindquist, R. A.; Moffat, J. CellProfiler: image analysis software for identifying and quantifying cell phenotypes. Genome biology 2006, 7 (10), R100.

22. Kamentsky, L.; Jones, T. R.; Fraser, A.; Bray, M.-A.; Logan, D. J.; Madden, K. L.; Ljosa, V.; Rueden, C.; Eliceiri, K. W.; Carpenter, A. E. Improved structure, function and compatibility for CellProfiler: modular high-throughput image analysis software. Bioinformatics 2011, 27 (8), 1179-1180.

23. Oppenheim, J. J. Cytokines: past, present, and future. International journal of hematology 2001, 74 (1), 3-8.

24. Salvemini, D.; Masini, E.; Pistelli, A.; Mannaioni, P. F.; Vane, J. Nitric oxide: a regulatory mediator of mast cell reactivity. Journal of cardiovascular pharmacology 1991, 17, S258\&hyhen.

25. Johnston, L. K.; Rims, C. R.; Gill, S. E.; McGuire, J. K.; Manicone, A. M. Pulmonary macrophage subpopulations in the induction and resolution of acute lung injury. American journal of respiratory cell and molecular biology 2012, 47 (4), 417-426.

26. Schif-Zuck, S.; Gross, N.; Assi, S.; Rostoker, R.; Serhan, C. N.; Ariel, A. Saturated-efferocytosis generates pro-resolving CD11blow macrophages: Modulation by resolvins and glucocorticoids. European journal of immunology 2011, 41 (2), 366-379.

27. Mehra, N. K.; Mishra, V.; Jain, N. A review of ligand tethered surface engineered carbon nanotubes. Biomaterials 2014, 35 (4), 1267-1283.

28. Thomassen, M. J.; Buhrow, L. T.; Connors, M. J.; Takao Kaneko, F.; Erzurum, S. C.; Kavuru, M. S. Nitric oxide inhibits inflammatory cytokine production by human alveolar macrophages. American Journal of Respiratory Cell and Molecular Biology 1997, 17 (3), 279-283.

29. Chen, E. H.; Grote, E.; Mohler, W.; Vignery, A. Cell-cell fusion. FEBS letters 2007, 581 (11), 2181-2193. 
30. Helming, L.; Gordon, S. Macrophage fusion induced by IL-4 alternative activation is a multistage process involving multiple target molecules. European journal of immunology 2007, 37 (1), 33-42.

31. Lander, H.; Sehajpal, P.; Levine, D.; Novogrodsky, A. Activation of human peripheral blood mononuclear cells by nitric oxide-generating compounds. The Journal of Immunology 1993, 150 (4), 1509-1516. 


\section{Chapter 5 : Nitric Oxide Releasing Materials as Potential Antivirals}

\subsection{Introduction}

The applications of $\mathrm{NO}$ as an antibacterial has been demonstrated from previous controlled NO releasing materials, and is the primary method for macrophages to kill foreign bacteria around a wound site through the formation of peroxynitrites ${ }^{1}$. This same mechanism can be applied to viruses as peroxynitrite byproducts will be able to react with viral proteins in the same manner. NO has already been proven to be a potent antiviral molecule, but the exact dosage over time has never been properly tested ${ }^{2}$. In past studies, an NO donor such as SNAP is added into solution at high concentrations to interact with the suspended viruses. This strategy has shown to inhibit virus replication by targeting viral protease in Coxsackievirus ${ }^{3}$. Specifically, NO was able to nitrosate the active cysteine sites on the viral protease enzymes, which reduced the overall functionality of the virus. Dengue virus was also observed in a separate study where the addition of SNAP was able to suppress viral RNA synthesis ${ }^{4}$. SNAP was added to culture medium at concentrations of $150 \mu \mathrm{m}$, which was replenished every 4 hours up to 8 hours after infection and then incubated for 40 hours.

Using NO as an antiviral poses the question of how it exactly is interacting with the viral structure as a whole - whether it is interacting with the viral envelope, the protein capsid, enzymatic molecules within the virus, or the actual DNA/RNA structure itself. $\mathrm{NO}$ as a radical molecule is capable of reacting with multiple types of functional groups to slightly alter these important structures of viruses as a method for disabling their ability to attach to cells and inject their genetic material to be replicated. NO has also been shown to alter DNA/RNA structures through nitrosative deamination of base pairs $^{5}$. Figure 5.1 shows how an NO donating material can affect a virus from normally functioning and replicating. This could be another mechanism for which viruses become 
deactivated after being exposed to NO. As there are multiple different classes of viruses, how NO interacts with each one of them is bound to be different.

NO Donating Material
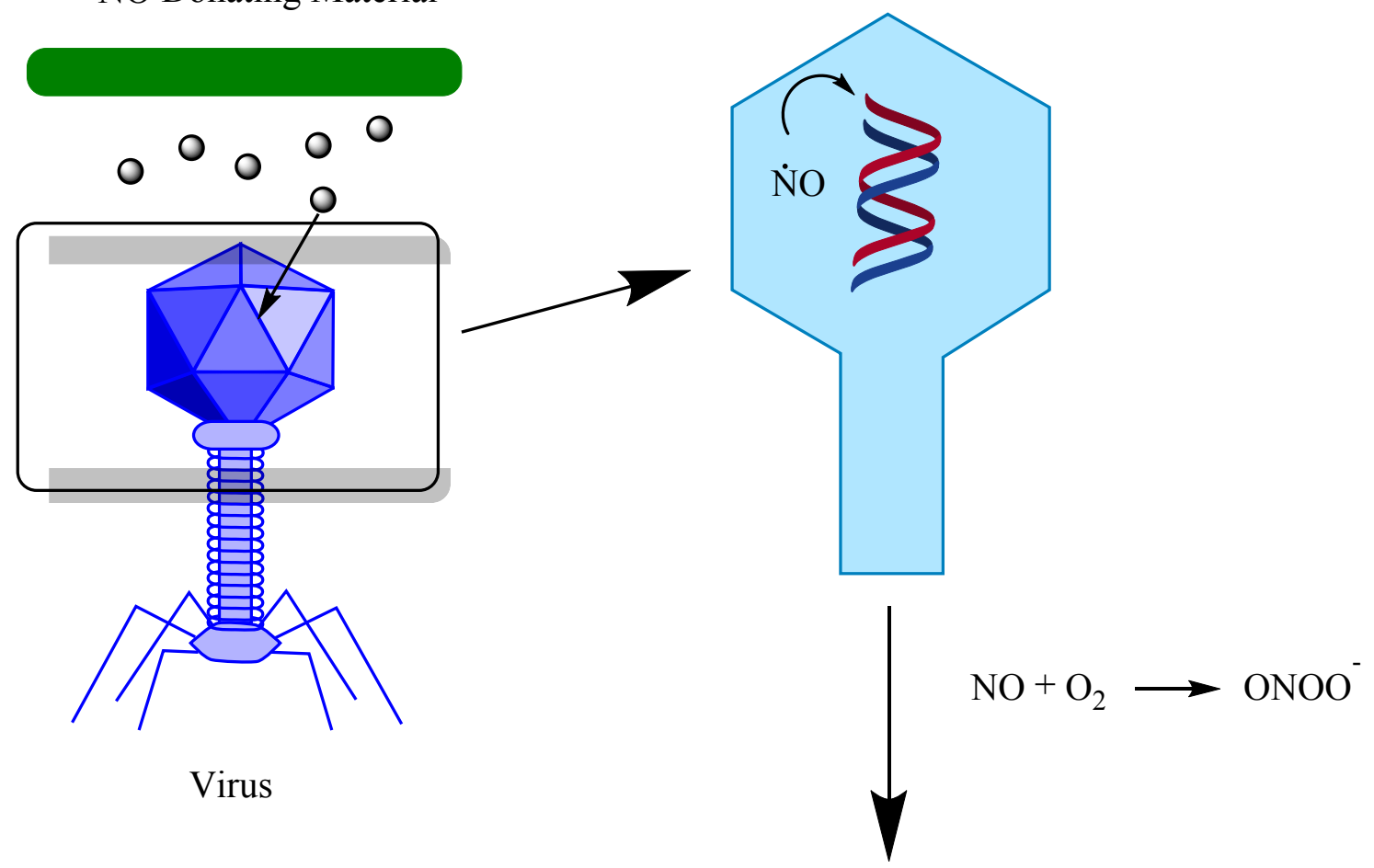

-Nitrosative deamination -Oxidative damage

Figure 5.1. Mechanism for DNA damage within viral species from an NO donating material.If NO does not react with the protein capsid layer, its peroxynitrite byproduct will be able to diffuse through and react with the DNA structures within. This leads to deamination of the base pairs in the DNA structure and causes oxidative damage, causing the virus to become unable to infect other cells.

The investigation of using $\mathrm{NO}$ as a potential antiviral was done through using controlled doses of NO over specific time intervals with SNAP-PDMS and SNAP-PVC. This gave a preliminary insight into how much $\mathrm{NO}$ is required to inactivate viruses from transfecting other vulnerable cells. The two viruses that were used were porcine parvovirus (PPV) and sindbis virus (SINV). PPV is a single stranded DNA (ssDNA) 
classified virus with no viral envelope while SINV is a single stranded RNA (ssRNA) classified virus which contains a viral envelope.

In these preliminary studies, SNAP-PDMS and SNAP-PVC were used as the material to deliver controlled amounts of NO over the course of 1 and 2 hours to PPV and SINV virus particles suspended in PBS. Once a threshold for the amount of NO over a specific time period can be identified, further experimenting can be done using higher doses over even shorter periods of time. For practical purposes in an environment where a virus is airborn, an NO releasing air filter could be developed to eliminate or deactivate an important viral function. This situation could require a high amount of NO being delivered at the interface of the filter, which the SNAP-HPAMAM material described in chapter 3 is more than capable of delivering. Shorter time studies can be done in the future using extreme amounts of NO delivery over the course of a few seconds using SNAP-HPAMAM once the NO threshold for deactivating a virus is discovered.

\subsection{Experimental Details}

\subsubsection{Materials}

The materials used to synthesize SNAP-PDMS were Hydroxy-terminated $2000 \mathrm{cSt}$ polydimethylsiloxane (PDMS) was purchased from Gelest, Inc. (Morrisville, PA, USA). 3-Aminopropyl trimethyoxysilane, dibutyltin dilaurate, cyclam, and toluene were obtained from Sigma-Aldrich Co. (St. Louis, MO, USA). Tert-butyl nitrite (90\% technical grade) was purchased from Acros Organics (Geel, Belgium).

\subsubsection{Synthesis of SNAP-PDMS}

The synthesis procedure followed a protocol described previously ${ }^{6} .1 .6 \mathrm{~g}$ of the hydroxyl-terminated PDMS is first dissolved in $8 \mathrm{~mL}$ of toluene. $0.3 \mathrm{~g}$ of 3aminopropyl trimethoxysilane and $2.4 \mathrm{mg}$ of dibutyltin dilaurate are then added to the solution and it was allowed to stir overnight to crosslink. This exposes primary amine 
groups that can then be reacted with NAP-thiolactone. $50 \mathrm{mg}$ of NAP-thiolactone is mixed with $2 \mathrm{~mL}$ of the crosslinked PDMS to form NAP-PDMS after allowing to stir for 24 hours. The newly exposed thiol group can then be reacted with t-butyl nitrite as is mentioned in the previous chapters.

\subsubsection{Experimental design}

A breadboard containing six blue, $470 \mathrm{~nm}$ LEDs connected to $130 \Omega$ resistors was constructed. PVC pipe (15 mm OD, $12 \mathrm{~mm}$ ID) was cut into $30 \mathrm{~mm}$ lengths and placed around the mounted LEDs. Set screws were placed $10 \mathrm{~mm}$ from the top of the PVC sections for the samples to rest on. SNAP-PVC and SNAP-PDMS were used as the NO releasing polymers for testing. The polymer samples were cast into films in the bottom of $2 \mathrm{~mL}$ glass vials. Due to the shape of the bottom of the vials, multiple layers were cast to ensure the final polymer product does not dry in a concave shape. A final layer of RTV-3140 (medical grade silicone rubber) was placed on top of the SNAP-PDMS layer to ensure no unwanted ion diffusion occurs while a layer of cellulose acetate was cast on the SNAP-PVC films.

For testing, the viral solutions were suspended in PBS and placed into the vials containing the bottom coated polymers. The LEDs were then turned on to trigger NO release from the polymer films into the viral solution for 1 and 2 hours. After the NO treatment, the viral solutions were transferred into microcentrifuge tubes and refrigerated until they were used for MTT assay analysis. The cells to be infected with the viral solutions were PK13 (ATCC CRL-6489) pig epithelial cells.

\subsubsection{Nitric oxide measurements}

NO detection was recorded in real time from the nitric oxide analyzer for multiple trials using the same setup that was being tested in the actual virus trials to accurately demonstrate the NO diffusion from the polymer films to the viruses suspended in PBS. 
This was done for both 1 and 2 hour trials. Ambient light testing was also done for control SNAP-PDMS samples where the LED was turned off.

\subsubsection{Virus activity measurements}

After being exposed to NO, cells were then administered with the treated virus solutions in culture. A live/dead assessment was then done on the treated and untreated cell cultures using an MTT assay.

Log reduction value (LRV) of the solution was calculated to determine the percentage of virus particles able to still infect cells. Complete inactivation of the virus $(99.99 \%)$ was calculated at an LRV of 4 .

\subsection{Results and Discussion}

\subsubsection{Nitric oxide profiles}

Identical polymers were cast into the $2 \mathrm{~mL}$ vials and were tested for NO release using the same setup used in the viral NO testing. The polymers were tested at the same time the virus samples were being tested to ensure the release profiles were as similar as possible. 


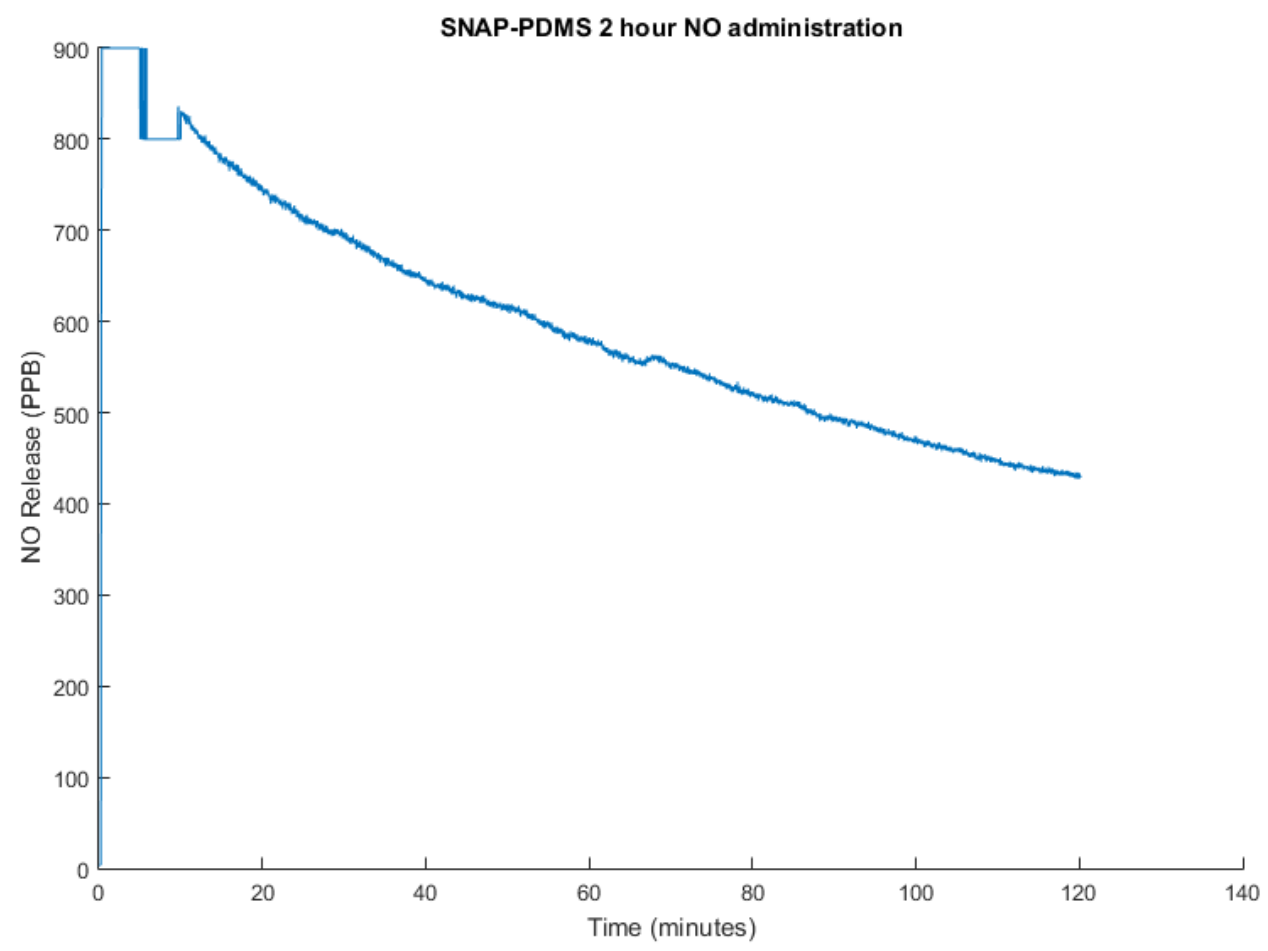

Figure 5.2. NO release profile of SNAP-PDMS over 2 hours when LED activated. The total amount of NO released over the 2 hour period was $0.270 \mu \mathrm{mol}$, and was proven to be enough NO to deactivate PPV replication.

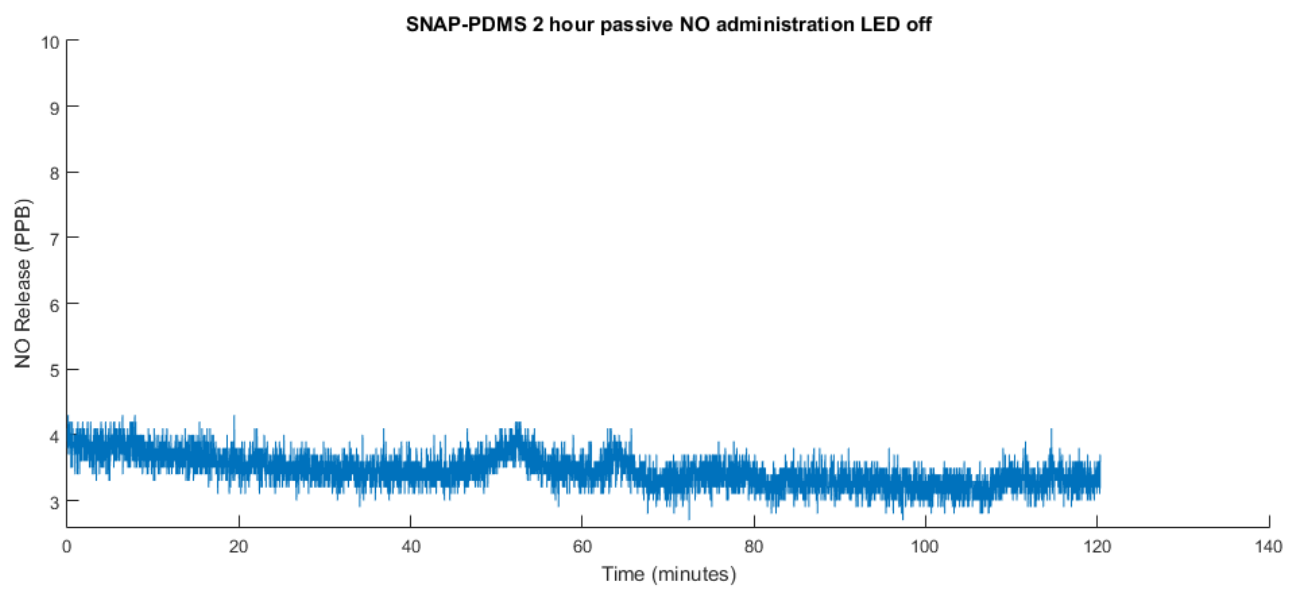

Figure 5.3. NO release profile of SNAP-PDMS passively with no LED over 2 hours. The amount of NO in the trials with the LED off was done in the scenario where there was viral removal with extremely low amounts of NO. Later experiments demonstrated that this low, passive amount of NO release had no effect on the viruses. 


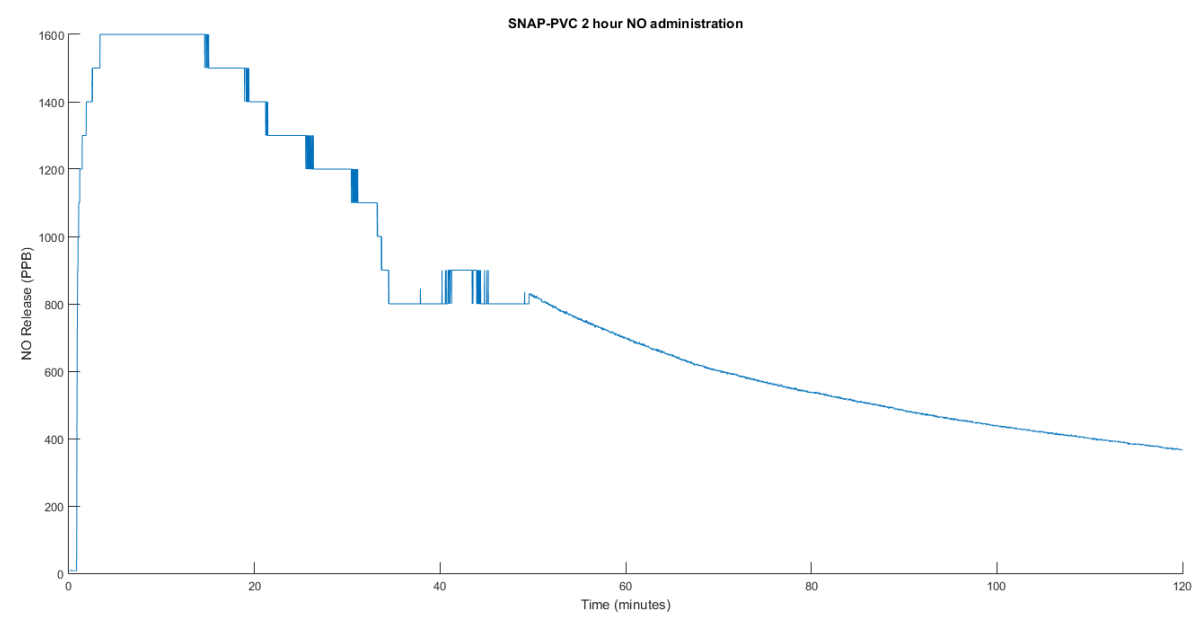

Figure 5.4. NO release profile from SNAP-PVC over a 2 hour time period. The release seen in the SNAP-PVC compounds was also shown to prevent PPV from infecting cultured cells.

\subsubsection{Virus Functionality}

Exposure to NO at both 1 and 2 hour release demonstrated complete deactivation of the viruses for SNAP-PVC and SNAP-PDMS. Comparing these preliminary results to other studies that have been done in the past show that the amount of SNAP that was previously being put into viral solutions were much higher than what is needed.

Although the viruses were unable to infect cells in culture after exposure to NO, a lower limit still needs to be discovered. Once this lower limit is determined, a more precise NO releasing material design can be made using materials like SNAP-HPAMAM. Depending on the amount of NO required over a period of time, a material can be tailored to have a certain amount of SNAP-HPAMAM blended in it to ensure the viruses are exposed to that level.

\subsubsection{Preliminary Data Collection}

SNAP-PVC was used as the first NO donating material to attempt to deactivate PPV in solution. The first trials that were done showed promising results of high viral removal after being exposed to both 1 and 2 hours of LED triggered SNAP-PVC. However, after 
further investigation, it was shown that the high NO release was actually causing the formation of nitric acid in the viral solutions, which drastically decreased the $\mathrm{pH}$. Even without the LED turned on, passive SNAP-PVC release from ion diffusion was causing a change in $\mathrm{pH}$.

\section{Log Removal for NO PPV trial}

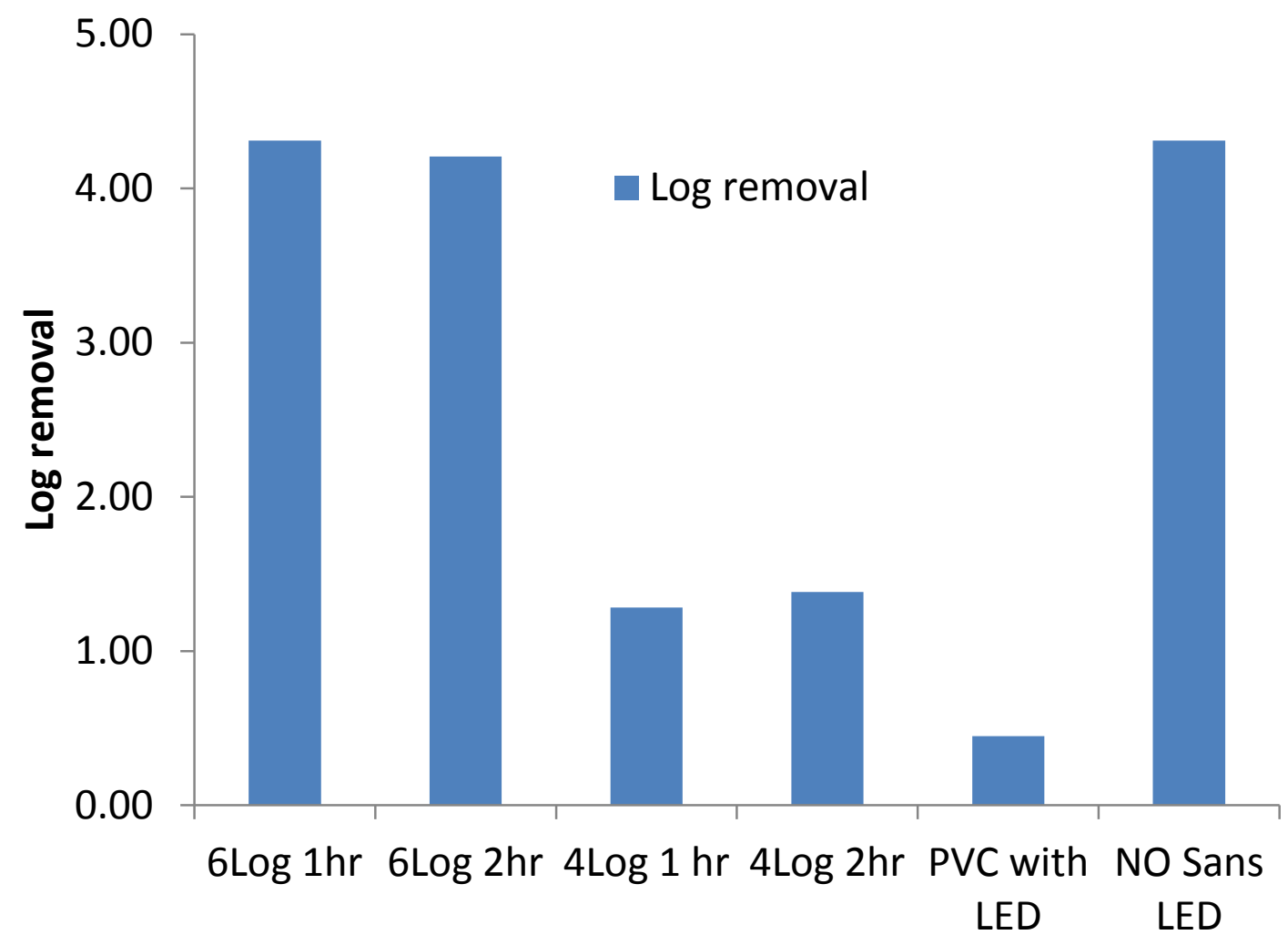

Figure 5.5. PPV removal using SNAP-PVC as the NO releasing polymer. The LED was turned on for 1 and 2 hour time points. Log 4 removal of the virus shows that $99.99 \%$ of the virus was deactivated or killed when administered to cell cultures, which was demonstrated for SNAP-PVC when the LED was on and off. The removal with the

LED off was due to the $\mathrm{pH}$ change seen from surface nitrites on the SNAP-PVC diffusing into solution to form nitric acid. Control PVC with the LED on and off showed no removal of the virus.

To solve the issue of passive $\mathrm{pH}$ change causing virus deactivation, the SNAP-PVC films were thoroughly rinsed with DI water before being tested with the viral solutions. This washes off any surface nitrites that may have lingered from the t-butyl nitrite 
nitrosation step that end up diffusing into solution causing the formation of nitric acid. After washing, the SNAP-PVC was then tested for NO release and ensured no $\mathrm{pH}$ change occurred before being tested. Tests proved 99.99\% removal of PPV with the administration of NO over a 1 hour and 2 hour period. Control PVC and SNAP-PVC with the LED turned off demonstrated no viral inactivation. No $\mathrm{pH}$ change was observed for any of the trials.

\section{Log Removal for NO PPV trial}

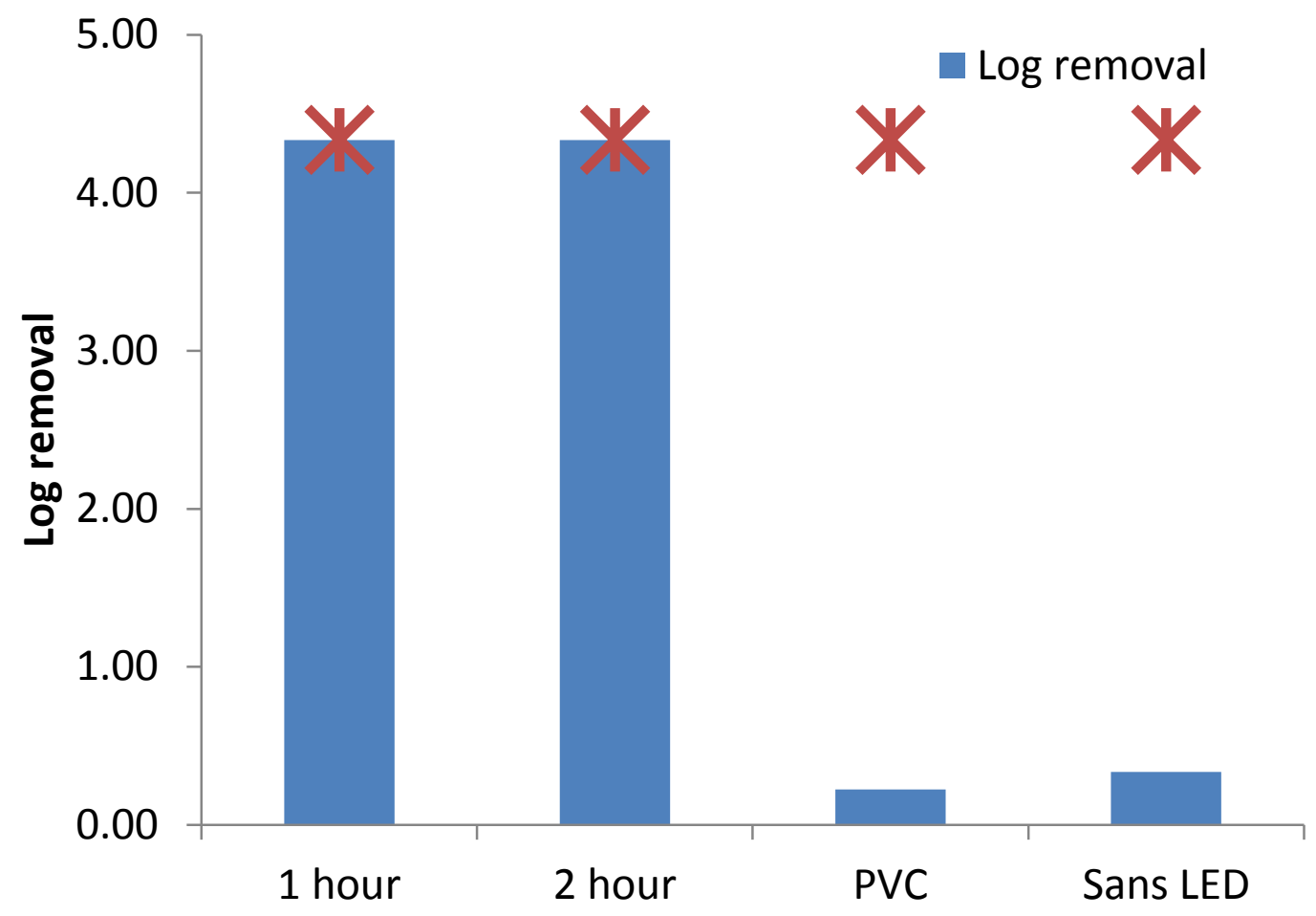

Figure 5.6. PPV removal using pre-washed SNAP-PVC as the NO releasing polymer. The LED was turned on for 1 and 2 hour time points. Log 4 removal of the virus shows that $99.99 \%$ of the virus was deactivated or killed when administered to cell cultures. Control PVC and SNAP-PVC with the LED off showed no removal of the virus. There was no $\mathrm{pH}$ change as well for the trials, proving $\mathrm{NO}$ was causing the removal.

\subsection{Conclusion}


The results presented show the potential of NO as a possible antiviral tool for future materials. The experiments described demonstrate how with enough time, a precise measurement on how much NO over a certain amount of time is needed to deactivate the infection potential of viral species. After this dose of NO is determined, it can be applied to real life situations where the spread of airborn viruses in closed locations is problematic.

\subsection{References}

1. Nablo, B. J.; Rothrock, A. R.; Schoenfisch, M. H. Nitric oxide-releasing solgels as antibacterial coatings for orthopedic implants. Biomaterials 2005, 26 (8), 917-924.

2. Marletta, M. A.; Yoon, P. S.; Iyengar, R.; Leaf, C. D.; Wishnok, J. S. Macrophage oxidation of L-arginine to nitrite and nitrate: nitric oxide is an intermediate. Biochemistry 1988, 27 (24), 8706-8711.

3. Fang, F. C. Perspectives series: host/pathogen interactions. Mechanisms of nitric oxide-related antimicrobial activity. Journal of Clinical Investigation 1997, 99 (12), 2818.

4. Croen, K. D. Evidence for antiviral effect of nitric oxide. Inhibition of herpes simplex virus type 1 replication. Journal of Clinical Investigation 1993, 91 (6), 2446.

5. Saura, M.; Zaragoza, C.; McMillan, A.; Quick, R. A.; Hohenadl, C.; Lowenstein, J. M.; Lowenstein, C. J. An antiviral mechanism of nitric oxide: inhibition of a viral protease. Immunity 1999, 10 (1), 21-28.

6. Takhampunya, R.; Padmanabhan, R.; Ubol, S. Antiviral action of nitric oxide on dengue virus type 2 replication. Journal of general virology 2006, 87 (10), 30033011. 
7. Lin, Y.; Huang, Y.; Ma, S.; Yeh, C.-T.; Chiou, S.; Chen, L.; Liao, C. Inhibition of Japanese encephalitis virus infection by nitric oxide: antiviral effect of nitric oxide on RNA virus replication. Journal of virology 1997, 71 (7), 5227-5235.

8. Burney, S.; Caulfield, J. L.; Niles, J. C.; Wishnok, J. S.; Tannenbaum, S. R. The chemistry of DNA damage from nitric oxide and peroxynitrite. Mutation Research/Fundamental and Molecular Mechanisms of Mutagenesis 1999, 424 (1), 37-49.

9. Wink, D. A.; Kasprzak, K. S. DNA deaminating ability and genotoxicity of nitric oxide and its progenitors. Science 1991, 254 (5034), 1001.

10. Gierke, G. E.; Nielsen, M.; Frost, M. C. S-Nitroso-N-acetyl-D-penicillamine covalently linked to polydimethylsiloxane (SNAP-PDMS) for use as a controlled photoinitiated nitric oxide release polymer. Science and Technology of Advanced Materials 2011, 12 (5), 055007. 


\section{Chapter 6 : Concluding Remarks and Future Direction}

\subsection{Summary of Findings}

The utilization of NO is still a growing field and more applications are still being discovered. By understanding how our body synthesizes and releases NO broadens the potential for NO donating materials to mimic some of these beneficial effects. Whether it is killing off bacteria through the iNOS synthetic route of macrophages or allowing our vasculature to maintain a healthy blood pressure through the production of NO from endothelial cells, NO donors will strongly impact the medical field.

The synthesis of a covalent bound NO donor to PVC to create a SNAP-PVC compound opens up a variety of opportunities for how PVC is used in the medical field. Being one of the most commonly used polymers in the medical industry, there is huge potential for replacing common PVC based devices while expanding its uses into other blood and tissue contacting devices such as catheters.

Using a similar hyperbranched molecular synthesis approach that is seen in dendrimers, a hyperbranched SNAP based polyamidoamine was successfully synthesized and shown to be extremely stable when isolated as a solid. The synthesized SNAP-HPAMAM is one of the highest capacity NO donating materials by mass when compared to the variety of other NO donating materials. Although highly branched NO releasing dendrimers have been synthesized previously, the amount of cost and time it takes to reach that level is extreme. Using hyperbranched chemistry greatly cuts down on both of these issues while still maintaining a high NO capacity.

These two materials were then able to demonstrate their capabilities of using the beneficial effects of NO by warding off unwanted inflammation when implanted subcutaneously. The PVC blended with SNAP-HPAMAM ended up performing better than SNAP-PVC due to its NO releasing longevity. Overall, both materials had less 
aggressive macrophage infiltration, less fibrous encapsulation of the implant, and less FBGC fusion occurrences at the tissue-implant interface.

Preliminary findings of NO's effect on viruses proved to be very promising. The setup being used is more effective than what has previously been done when investigating the concentrations needed to deactivate viral compounds. Over a period of 1 and 2 hours, both SNAP-PVC and SNAP-PDMS polymers were able to deliver enough NO to obtain an LRV of 4 . The method of having a polymeric system releasing NO into solution is a much more precise method than simply adding SNAP into a solution containing your target virus.

\subsection{Future directions}

While covalently binding SNAP to the backbone of PVC was successful, its overall capacity was lower than expected. This was due to the complications with attaching a difunctional compound to the backbone of a polymer. In this scenario, crosslinking is virtually unavoidable, which limits the loading capacity potential to PVC. To improve on this, a different strategy will need to be implemented. One possible suggestion is to use a diamino compound that has one of the primary amine groups protected. Once the free primary amine group is attached to the polymer backbone, it will be unable to crosslink with other polymer chains. The material can then be deprotected, exposing a primary amine group for NAP-thiolactone to attach to. This could greatly increase the NO capacity potential, but would also increase the cost to synthesize.

Although the NO capacity in SNAP-HPAMAM was shown to be very high, there is still room for improvement to increase it. One of the major issues seen during the synthesis was attaching the NAP-thiolactone to the material efficiently. If this problem could be overcome, the storage capability would increase dramatically.

Future studies for subcutaneous implants using SNAP-HPAMAM could be done where more of the NO donor is loaded onto other types of polymers over longer periods of 
time. The study done in chapter 4 was to examine the acute to chronic inflammation transition and NO's role in how it can slow its progress. The amount of SNAPHPAMAM loaded into the polymer was also fairly small, as there is a risk of releasing too much NO too quickly and causing cell necrosis which I wanted to avoid. For long term implants that last for months, it would be interesting to see how inflammation progresses in other hydrophobic or slow degrading polymers like PLLA.

By being able to accurately track the NO being released from a polymer film into a solution containing a target virus, we are able to pin point the exact dosage of NO over what period of time is necessary to deactivate viruses. This sets a new precedent for how NO can be administered to these types of targets suspended in solution. More insight into how NO is altering the viruses will need to be done in future studies. This could be done by observing changes in capsid proteins using western blotting techniques or MALDI-TOF. If the DNA or RNA structures are being altered, PCR could be done to prove any nitrosative deamination of base pairs that could be occurring. 


\section{List of Appendices}

\section{Appendix A Supporting Information for Chapter 2}

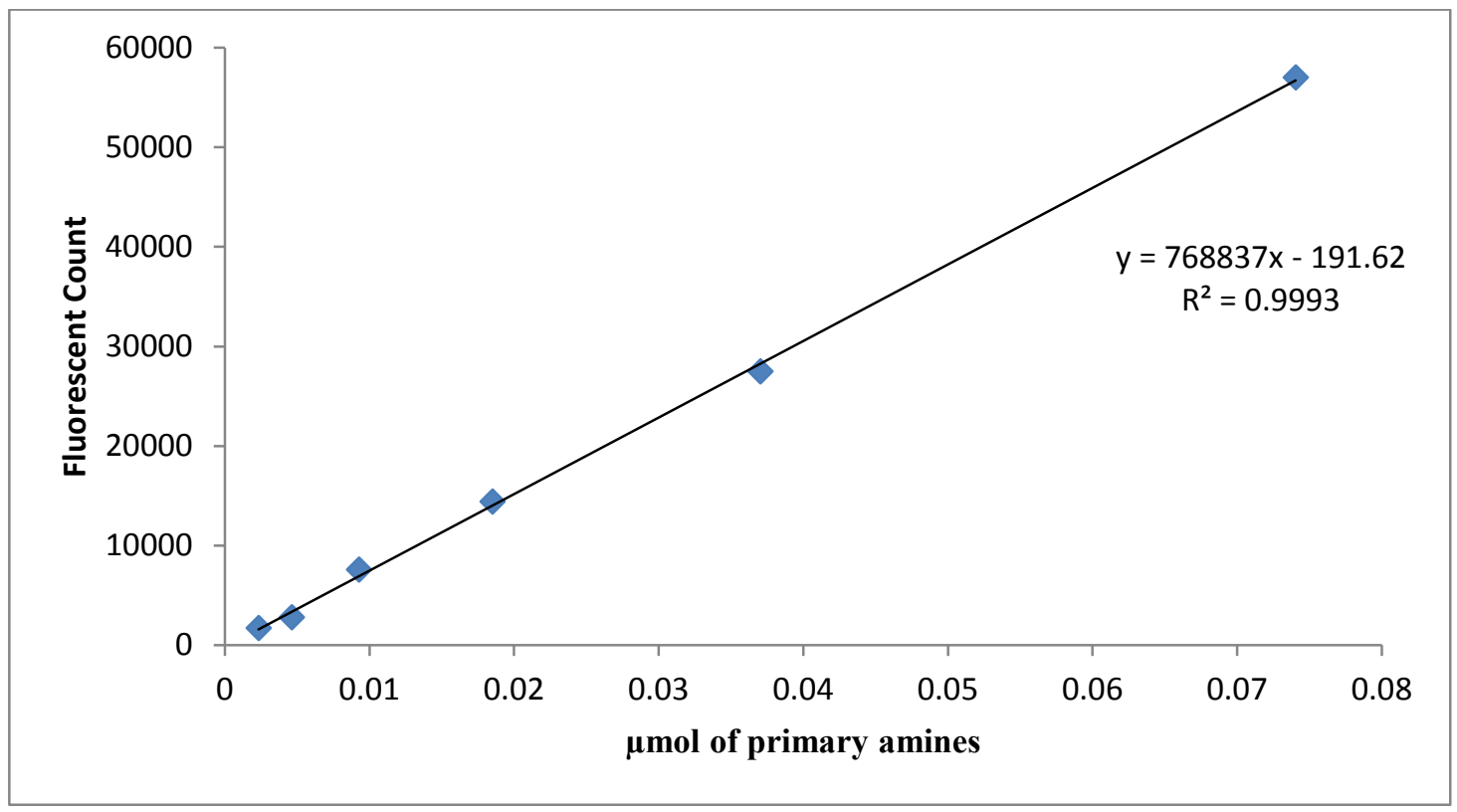

Figure A.1. Calibration curve of ATTO-TAG FQ test for amines using glycine- $\mathrm{HCl}$ as a standard. 


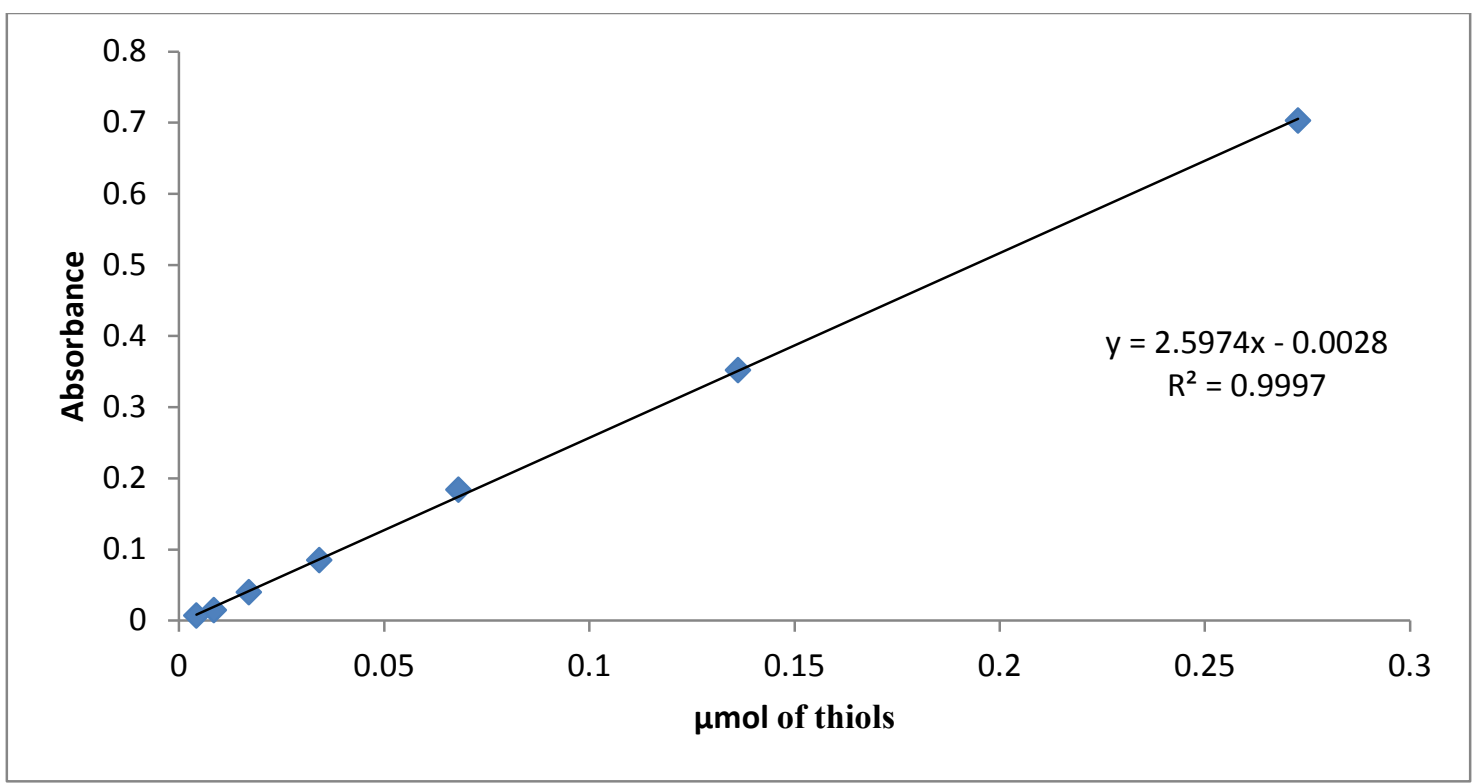

Figure A.2. Calibration curve of Ellman's test for thiols using cysteine as a standard.

Table A.1. CHN combustion analysis of the $\mathrm{PVC}-\mathrm{NH}_{2}$ polymer based on its reaction time with ethylenediamine. The table shows an increase in nitrogen content, but the ATTO-TAG FQ results show a decreased amount of primary amine sites once it reacts beyond 2 hours. This shows the possibility of crosslinking occurring between polymer chains as the reaction progresses. The ideal time point was found to be 2 hours.

\begin{tabular}{|c|c|}
\hline Sample & $\% \mathrm{~N}$ \\
\hline PVC 1 hour reaction time & 0.15 \\
\hline PVC 2 hour reaction time & 0.167 \\
\hline PVC 4 hour reaction time & 0.20 \\
\hline
\end{tabular}




\section{Appendix B Supporting Information for Chapter 3}

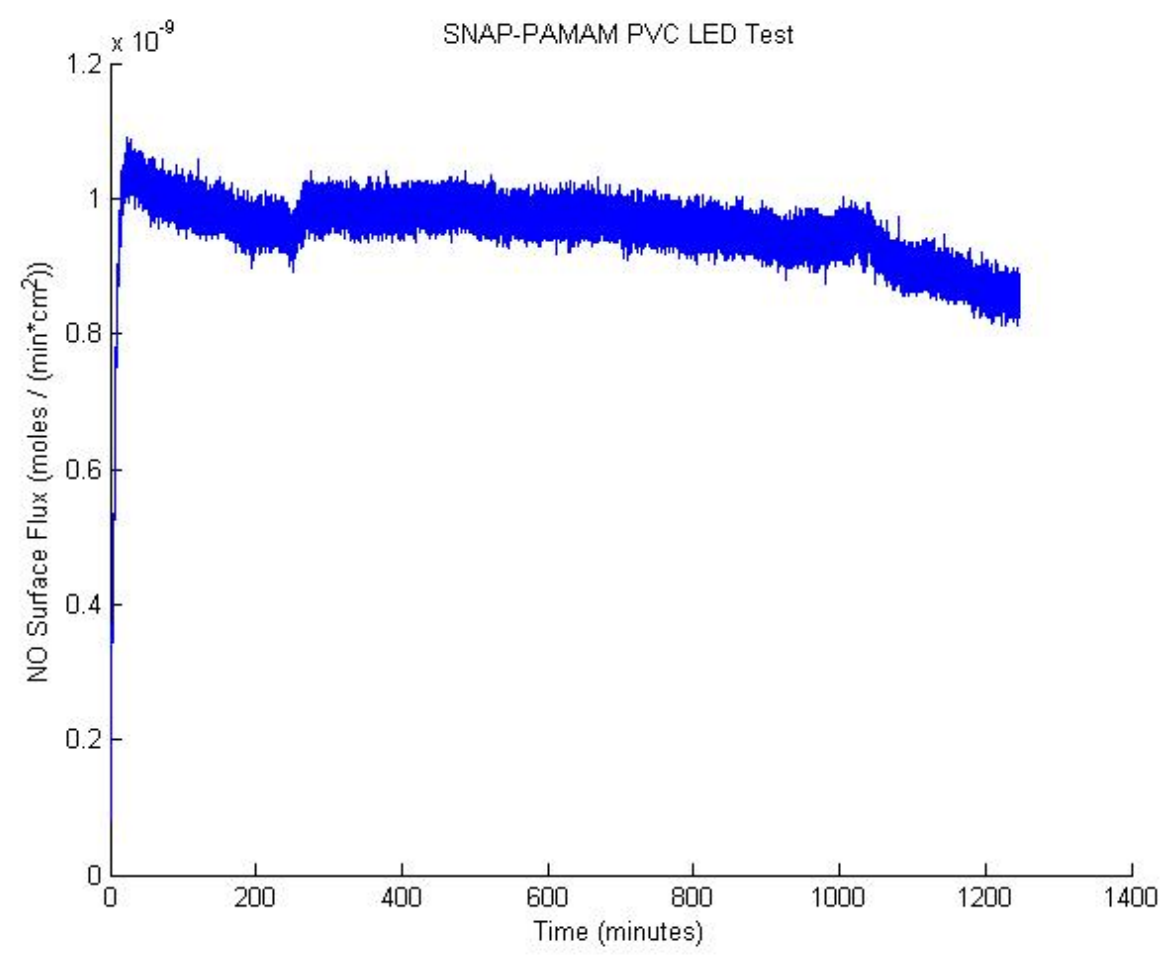

Figure B.1. SNAP-HPAMAM blended PVC under a $470 \mathrm{~nm} \operatorname{LED}(3 \mathrm{~V}, 150 \Omega)$.

Demonstrates SNAP-HPAMAM's ability to retain a controlled flux over a long period of time for in vitro testing. 


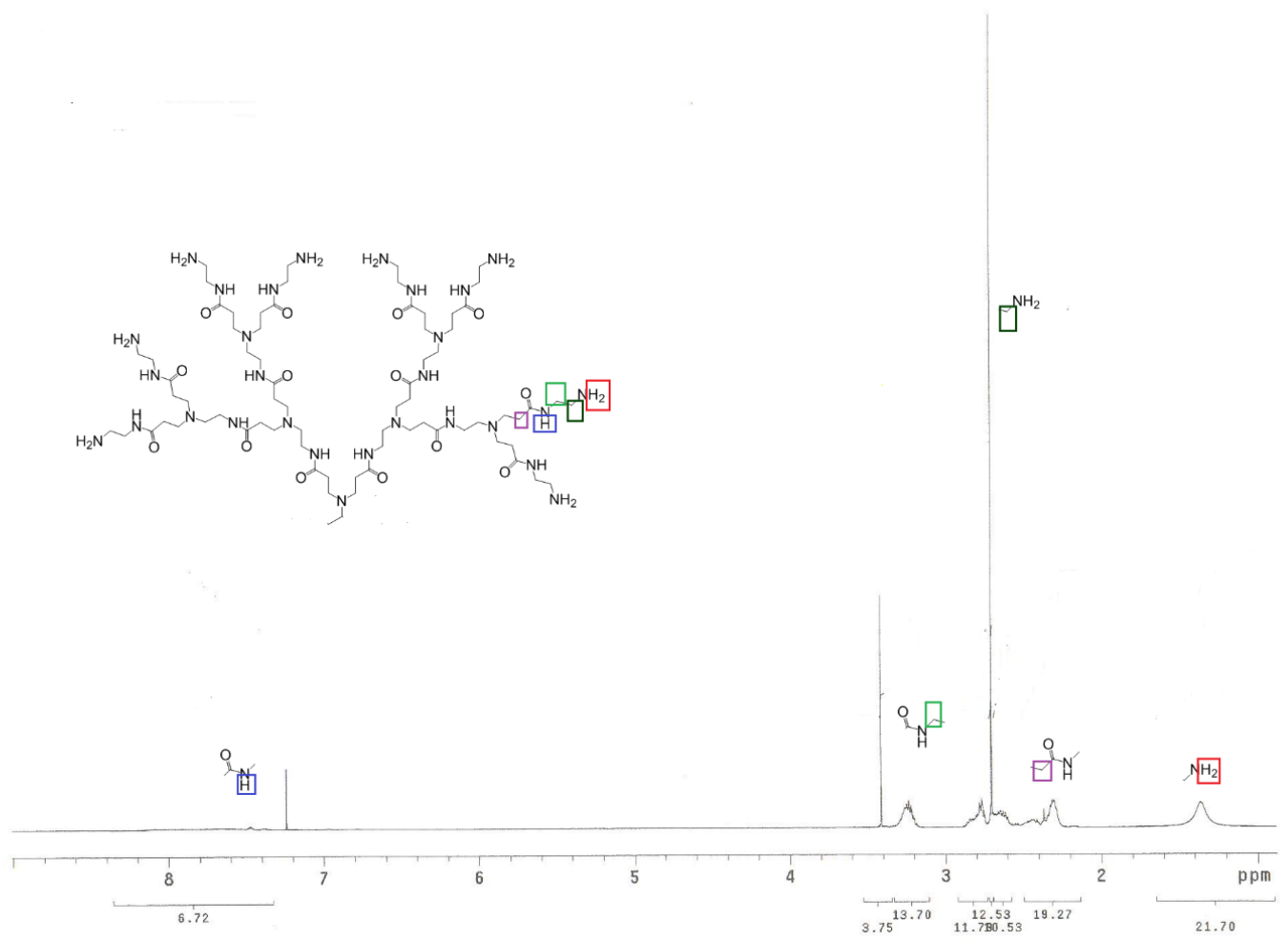

Figure B.2. ${ }^{1} \mathrm{H}$ NMR data of HPAMAM in $\mathrm{CDCl}_{3}$. 


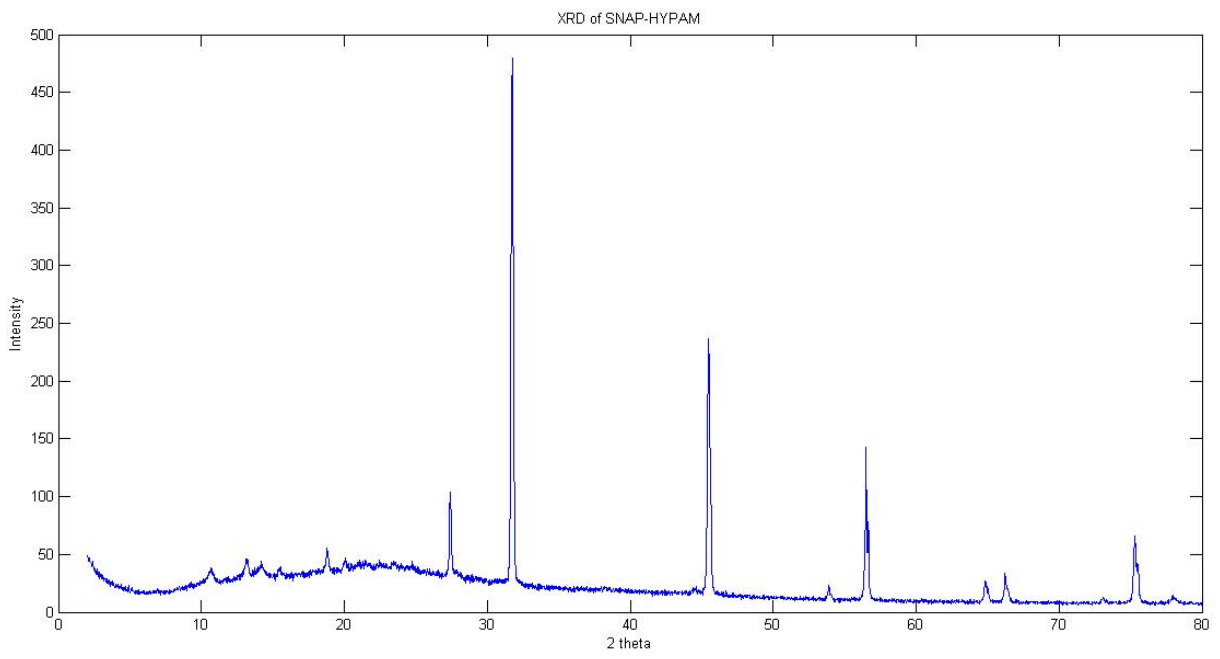

Figure B.3. XRD of SNAP-HPAMAM. Once nitrosated either by t-butyl nitrite or sodium nitrite, SNAP-HPAMAM displays unique crystalline features when isolated as seen in the XRD scan. 


\section{Appendix C}

\section{Supporting Information for Chapter 4}

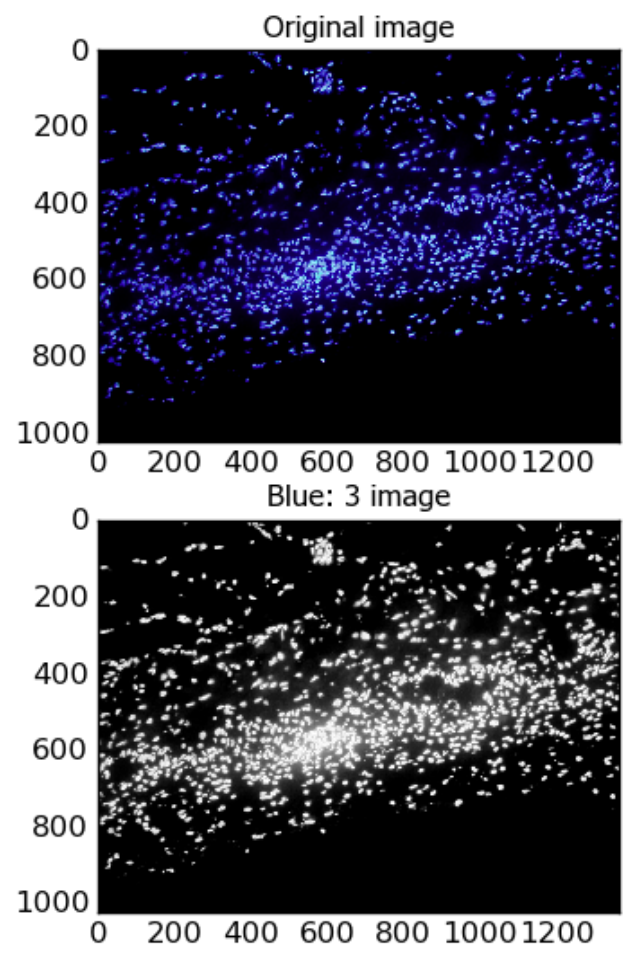

Figure C.1. CellProfiler example for cell nuclei (DAPI) marking. DAPI stained images are first converted to gray scale before counting analysis begins. 

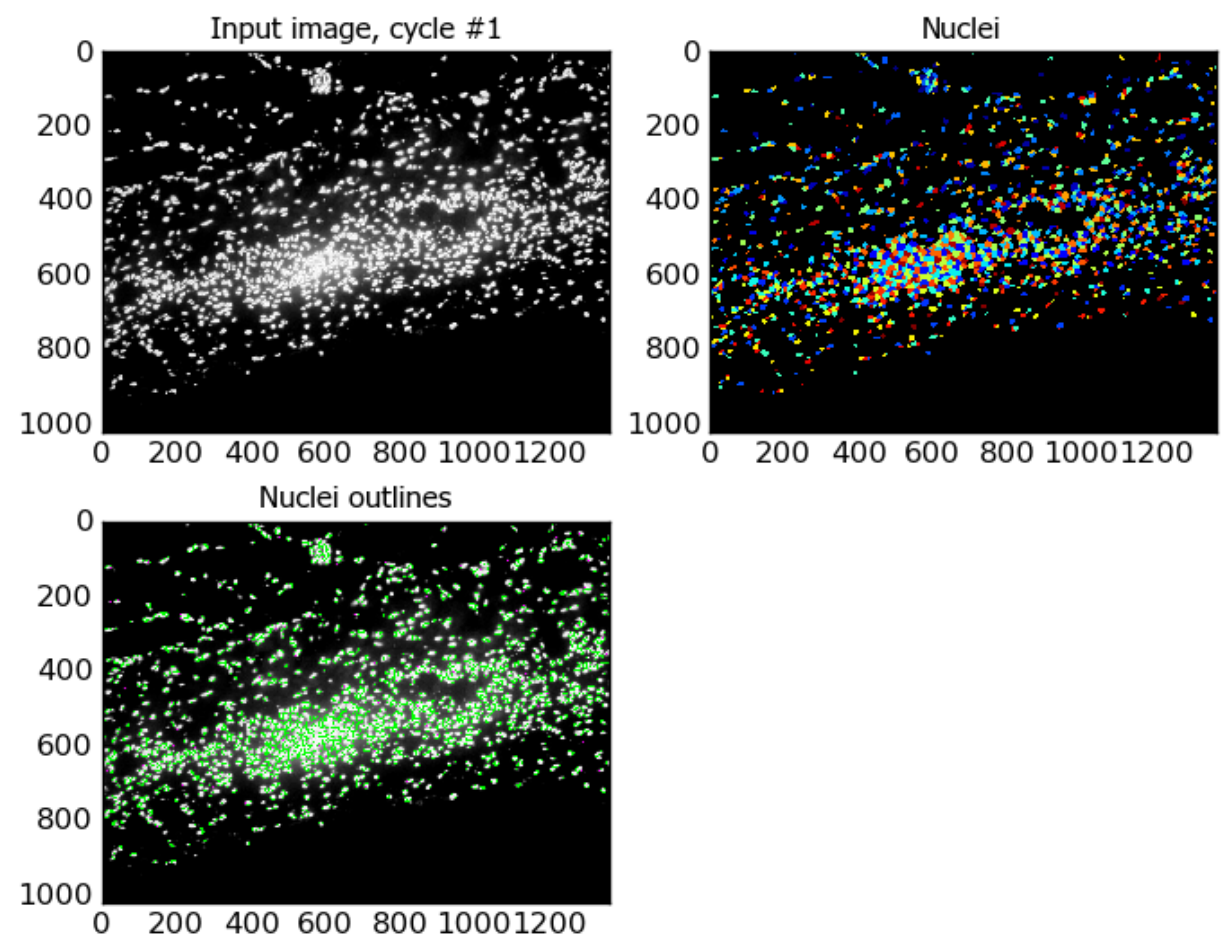

Figure C.2. CellProfiler counting analysis of DAPI stained cells. Parameters are set for the program to declare what specifies as a cell nuclei and what is not. Even in clumped areas where counting is difficult, the CellProfiler program is able to "declump" and count the objects based on signal intensity. 
Table C.1. Results and analysis of CellProfiler cell nuclei counting for Figure C.2. Displays the details given from the program when analyzing a specific image.

\begin{tabular}{|c|c|}
\hline Threshold & 0.365 \\
\hline \# of accepted objects & 1560 \\
\hline 10th pctile diameter & 7.7 pixels \\
\hline Median diameter & 13.0 \\
pixels
\end{tabular}


Figure C.3. CD163 stained SNAP-HPAMAM PVC image before CellProfiler processing. 


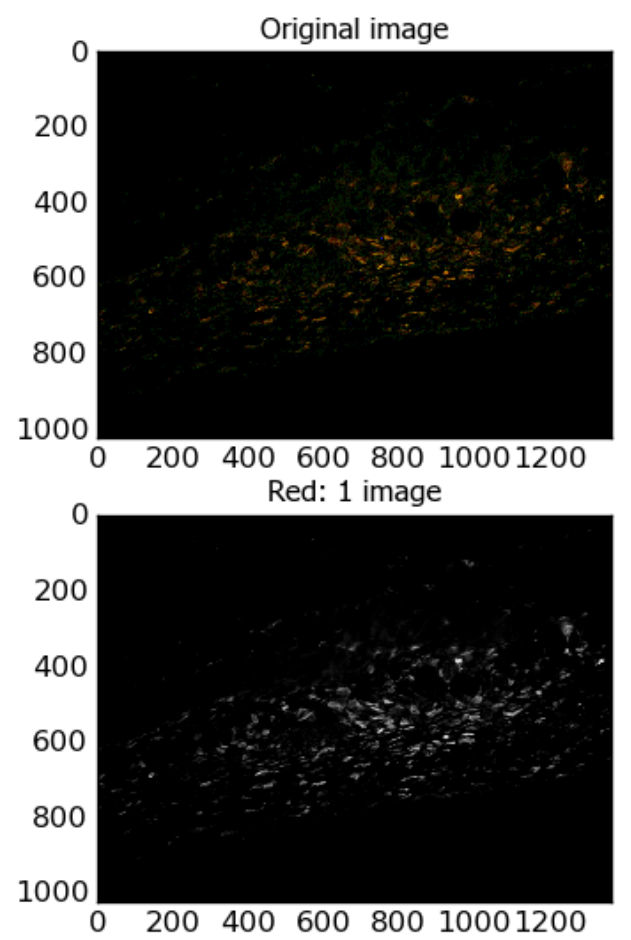

Figure C.4. CellProfiler conversion of Figure C.3 to grayscale for macrophage counting. 

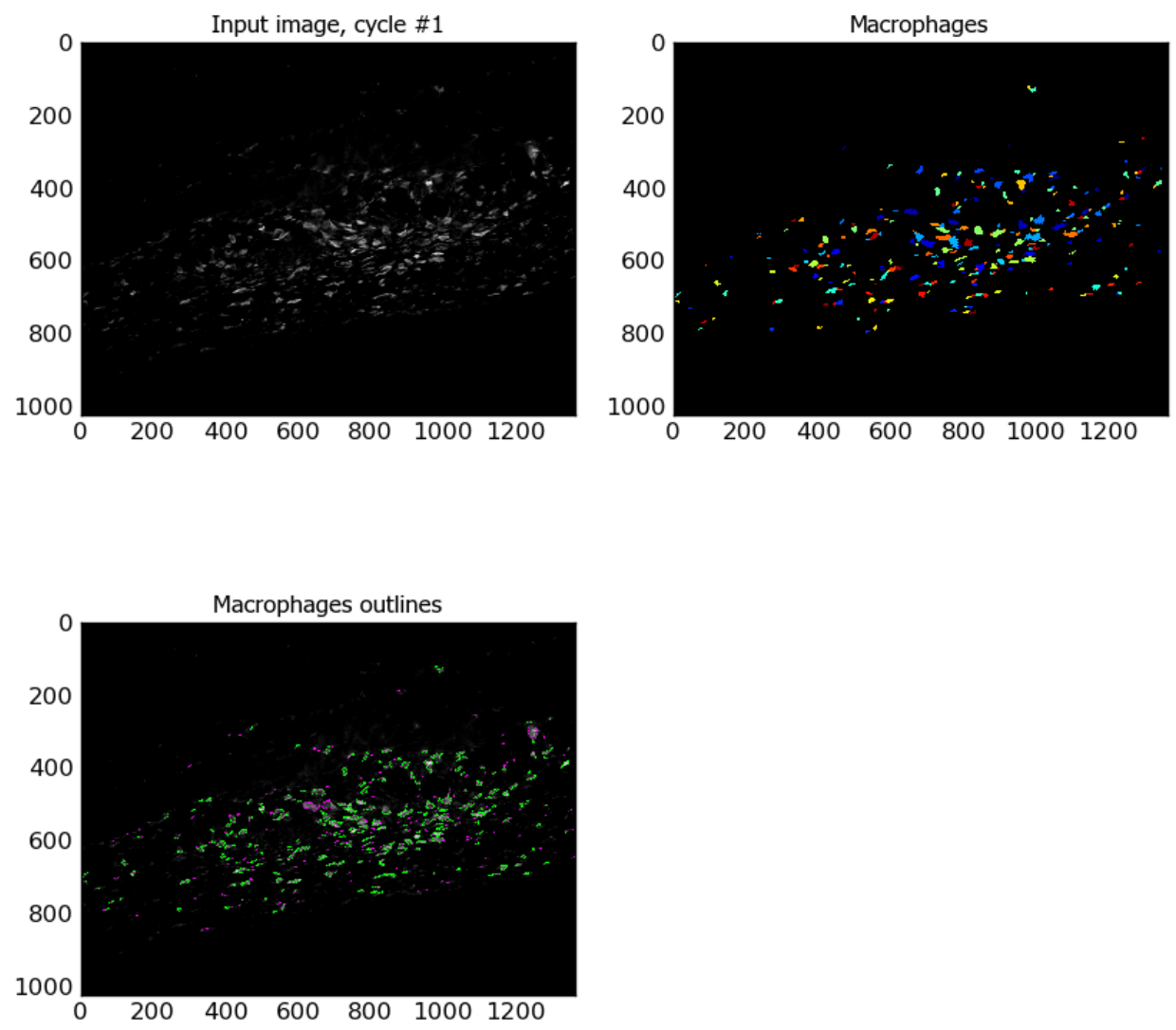

Figure C.5. CellProfiler counting and analysis of CD163 stained images. Setting parameters for macrophage stained images proved to be more difficult as some of the antibodies (CD163 and CD11b) mark membrane proteins. This meant that declaring the macrophage size was dependent on how much of the surface proteins were actually positively marked. 
Table C.2. Results and analysis of CellProfiler cell nuclei counting for Figure C.5.

\begin{tabular}{|c|c|}
\hline Threshold & 0.093 \\
\hline \# of accepted objects & 254 \\
\hline 10th pctile diameter & 7.7 pixels \\
\hline Median diameter & 10.5 \\
pixels \\
\hline 90th pctile diameter & 18.9 \\
pixels \\
\hline Area covered by objects & $2.40 \%$ \\
\hline Thresholding filter size & 1 \\
\hline LoG threshold & 0.4 \\
\hline LoG filter diameter & 10 \\
\hline Declumping smoothing filter & \\
\hline size & 4.7 \\
\hline Maxima suppression size & 4.7 \\
\hline
\end{tabular}




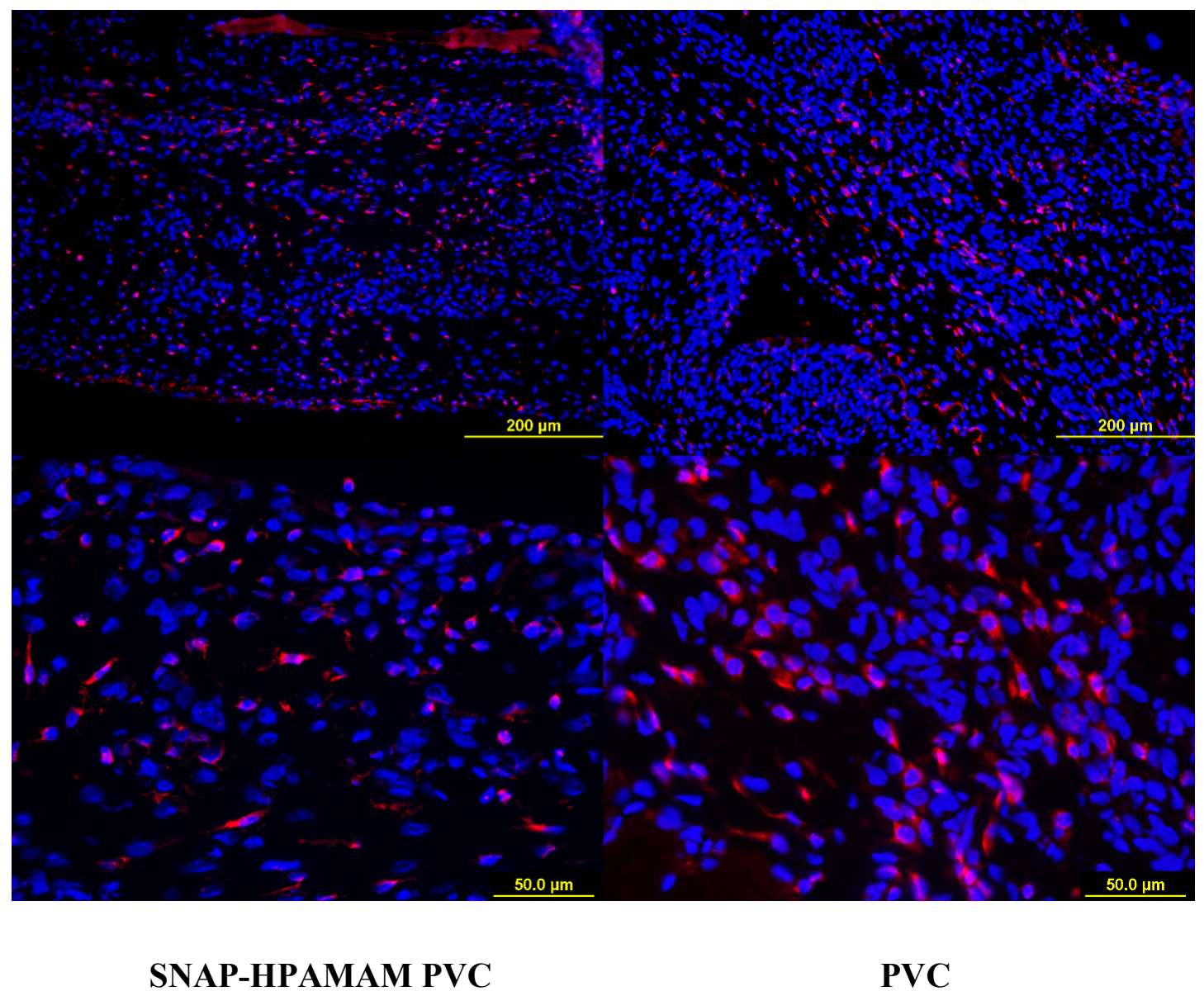

Figure C.6. CD68 macrophage marker for SNAP-HPAMAM PVC (left column, top 200x, bottom 600x) implanted films and PVC control (right column, top 200x, bottom 600x). Demonstrates NO's ability in the SNAP-HPAMAM form to lower the overall macrophage response to the implants along with lowering the cell density infiltration surrounding the implant. 


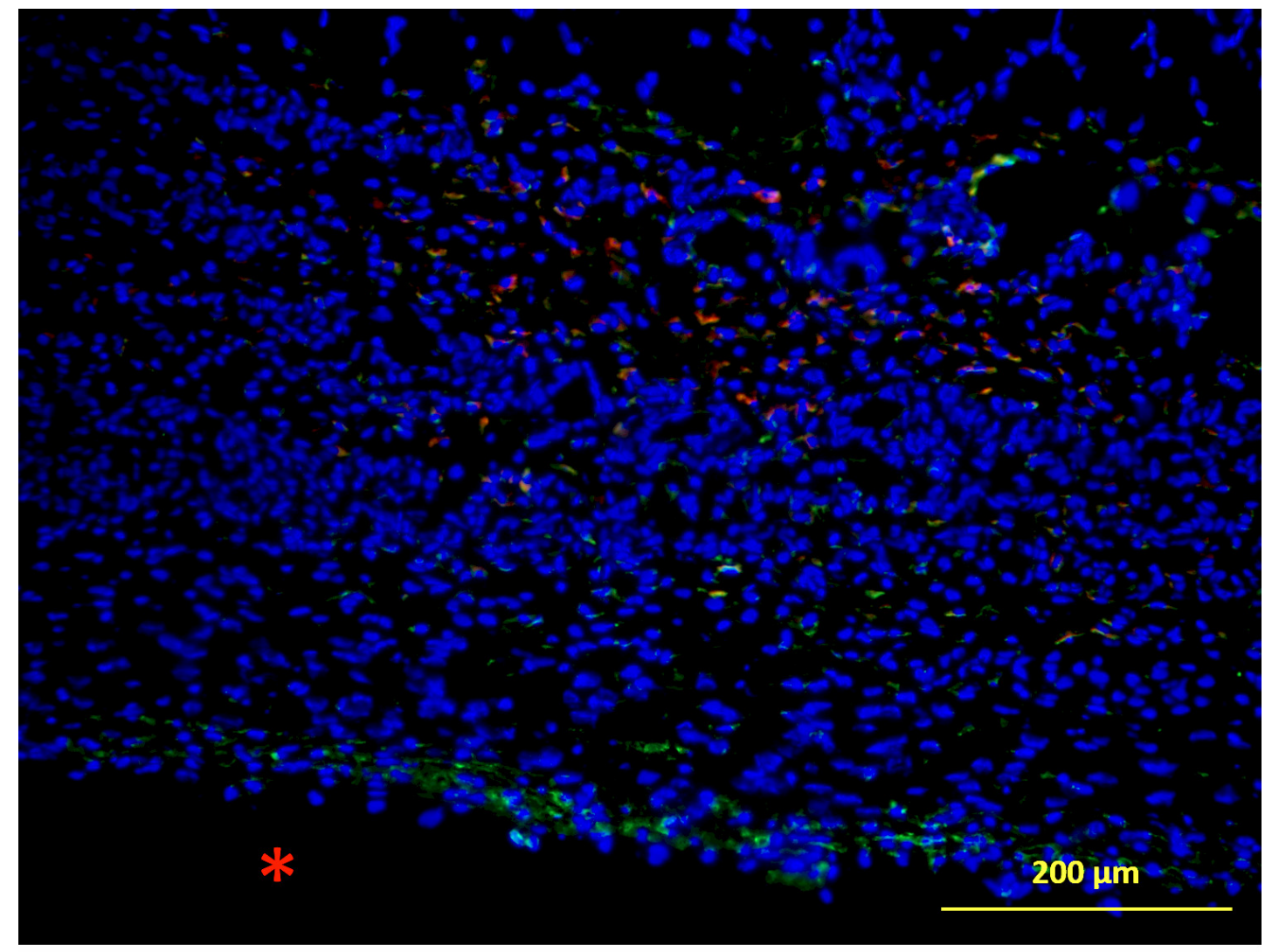

Figure C.7. 200x image of 15 day SNAP-HPAMAM PVC section stained with CD163 (red) and CD68 (green). This visualizes the location of M2 macrophages with respect to M1 macrophages. The M2 macrophages are closer to the adipose tissue, regulating the inflammation and migration of subsequent macrophages to the implant site. The M1 macrophages are mainly seen at the tissue-implant interface. Implant area is labeled with a red star. 


\section{Appendix D Supporting Information for Chapter 5}

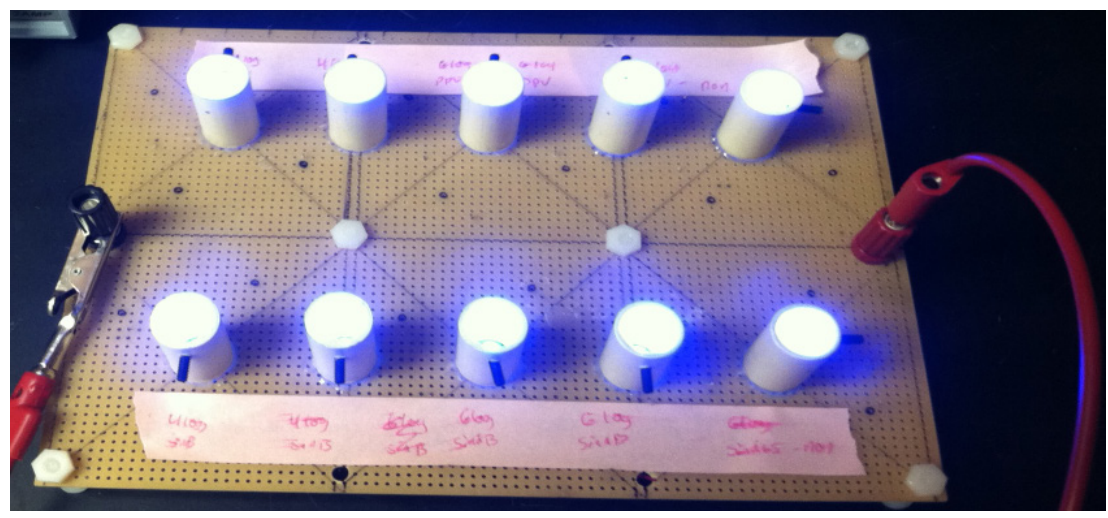

Figure D.1. Lightboard setup used for initiating NO release from vials containing SNAP based polymers.

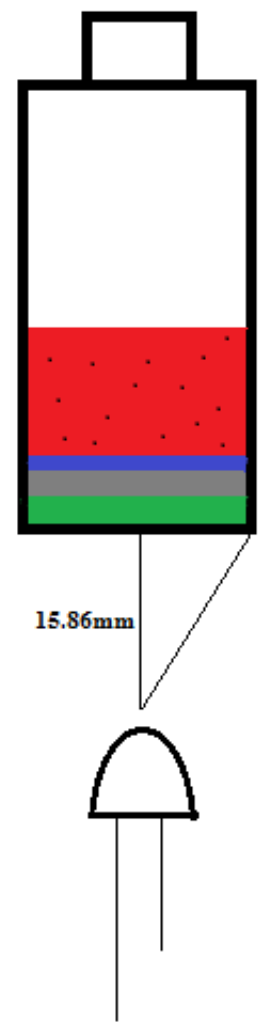


Figure D.2. Description of how the LED-virus setup interacts. A three layered system was initially used where the bottom layer (green) is the controlled NO releasing polymer layer, the middle layer (gray) is the control polymer layer to prevent cation diffusion into the SNAP polymer system, and the top layer (blue) is a cellulose layer to prevent the virus particles from attaching to the polymer surface. The LED's being used are VAOL-5GSBY4 5GSBY4 $(\lambda=460 \mathrm{~nm})$ and will be placed approximately $16 \mathrm{~mm}$ below the virus containing vial to make sure the 30 degree cone angle covers the entire $8.50 \mathrm{~mm}$ diameter film being cast. 